\title{
Modeling of Utility Distribution Feeder in OpenDSS with Steady State Impact Analysis of Distributed Generation
}

\author{
Vaidyanath Ramachandran \\ West Virginia University
}

Follow this and additional works at: https://researchrepository.wvu.edu/etd

\section{Recommended Citation}

Ramachandran, Vaidyanath, "Modeling of Utility Distribution Feeder in OpenDSS with Steady State Impact Analysis of Distributed Generation" (2011). Graduate Theses, Dissertations, and Problem Reports. 371. https://researchrepository.wvu.edu/etd/371

This Thesis is protected by copyright and/or related rights. It has been brought to you by the The Research Repository @ WVU with permission from the rights-holder(s). You are free to use this Thesis in any way that is permitted by the copyright and related rights legislation that applies to your use. For other uses you must obtain permission from the rights-holder(s) directly, unless additional rights are indicated by a Creative Commons license in the record and/ or on the work itself. This Thesis has been accepted for inclusion in WVU Graduate Theses, Dissertations, and Problem Reports collection by an authorized administrator of The Research Repository @ WVU. For more information, please contact researchrepository@mail.wvu.edu. 


\title{
Modeling of Utility Distribution Feeder in OpenDSS with Steady State Impact Analysis of Distributed Generation
}

\author{
Vaidyanath Ramachandran \\ Thesis submitted to the \\ College of Engineering and Mineral Resources \\ at West Virginia University \\ in partial fulfillment of the requirements \\ for the degree of \\ Master of Science \\ in \\ Electrical Engineering \\ Dr. Sarika Khushalani Solanki, Ph.D., Chair \\ Dr. Jignesh Solanki, Ph.D. \\ Prof. Ali Feliachi, Ph.D. \\ Lane Department of Computer Science and Electrical Engineering \\ Morgantown, West Virginia \\ 2011
}

Keywords: Distribution Systems Modeling, OpenDSS, PV Integration, Three Phase Unbalanced Distribution power flow.

Copyright 2011 Vaidyanath Ramachandran 


\section{ABSTRACT \\ Modeling of Utility Distribution Feeder in OpenDSS and Steady State Impact analysis of Distributed Generation \\ Vaidyanath Ramachandran}

With the deregulation of the electric power industry and the advancement of new technologies, the attention of the utilities has been drawn towards adopting Distributed Generation (DG) into their existing infrastructure. The deployment of DG brings ample technological and environmental benefits to the traditional distribution networks. The appropriate sizing and placement of DGs which generate power locally to fulfill consumer demands, helps to reduce power losses and avoid transmission and distribution system expansion.

The primary objective of this thesis is to model a utility distribution feeder in OpenDSS. Studies are conducted on the data obtained from American Electric Power utility. This thesis develops models for $12.47 \mathrm{kV}$ (medium voltage) distribution feeders in OpenDSS by utilizing the existing models in CYMDIST. The model conversion is achieved by a detailed one-to-one component matching approach for multi phased lines, conductors, underground cables, loads, regulators and capacitor banks. The power flow results of OpenDSS and CYMDIST are compared to derive important conclusions.

The second major objective is to analyze the impacts of DG on distribution systems and two focus areas are chosen, namely: effect on voltage profiles and losses of the system and the effects on power market operation. To analyze the impacts of DG on the distribution systems, Photovoltaic (PV) system with varying penetration levels are integrated at different locations along the developed feeder model. PV systems are one of the fastest growing DG technologies, with a lot of utilities in North America expressing interest in its implementation. Many utilities either receive incentives or are mandated by green-generation portfolio regulations to install solar PV systems on their feeders. The large number of PV interconnection requests to the utilities has led to typical studies in the areas of power quality, protection and operation of distribution feeders. The high penetration of PV into the system throws up some interesting implications for the utilities. Bidirectional power flow into a distribution system, (which is designed for one way power flow) may impact system voltage profiles and losses. In this thesis, the effects of voltage unbalance and the losses of the feeder are analyzed for different PV location and penetration scenarios.

Further, this thesis tries to assess the impact of DG on power market operations. In a deregulated competitive market, Generation companies (Genco) sell electricity to the Power exchange (PX) from which large customers such as distribution companies (Disco) and aggregators may purchase electricity to meet their needs through a double sided bidding system. The reliable and efficient operation of this new market structure is ensured by an independent body known as the Independent System Operator (ISO). Under such a market structure, a particular type of unit commitment, called the Price Based Unit Commitment (PBUC) is used by the Genco to determine optimal bids in order to maximize its profit. However, the inclusion of intermittent DG resources such as wind farms by the Gencos causes uncertainty in PBUC schedules. In this research, the effects of intermittency in the DG resource availability on the PBUC schedule of a Genco owning a distribution side wind farm are analyzed. 


\section{DEDICATION}

I would like to dedicate this thesis to my parents, Nirmala Ramachandran and V. Ramachandran for believing in me and always being there for me and for making me what I am today.

$\&$

My adorable aunt Shyamala Jagannathan and uncle S.N. Jagannathan, for making Morgantown a home away from my home! 


\section{ACKNOWLEDGEMENTS}

At this juncture, I have understood that the support and encouragement of a number of people around has helped me to complete my Masters degree successfully. First of all, I would like to thank my esteemed mentor and advisor, Dr. Sarika Khushalani Solanki. I owe her so much as she has given me a chance to work in the field of power systems. She taught me much of what I know today and guided me throughout my research work. I would also like to extend my sincere thanks to Dr. Jignesh Solanki, who has constantly motivated me to achieve more. I would also like to thank rest of my thesis committee, Dr. Ali Feliachi and Dr. Muhammad A Choudhry, for their support and advice. I extend the appreciation to Roger Dugan of EPRI for all help extended to me with regards to modeling in OpenDSS. This work would not have been completed without the support and cooperation of Tom Weaver and Joe Myers of American Electric Power utility.

I would also like to express my thanks to my friends in the lab for the healthy discussions regarding my research work. I have been fortunate enough to have a great group of friends at WVU. I would like to thank all of them for making my master's life joyful and memorable. Specially, I would like to thank my best friend and roommate, Naveen, who was always there for me when I needed moral support.

I would like to thank my family for their unending support and love from childhood to now. Without their support I would not have made it this far. My humble prayer goes to the almighty god for being with me as a guiding light throughout my life. 


\section{CONTENTS}

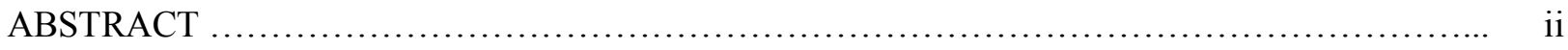

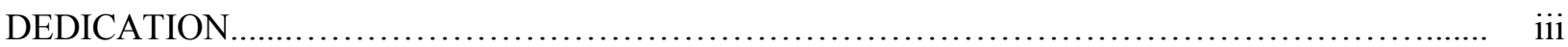

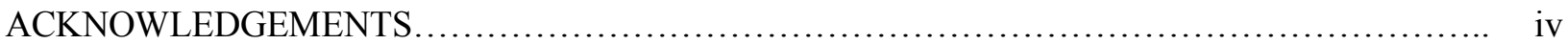

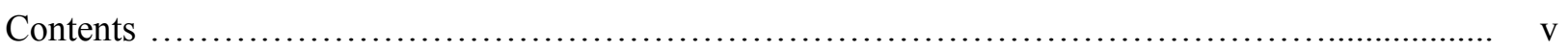

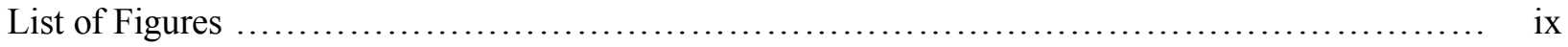

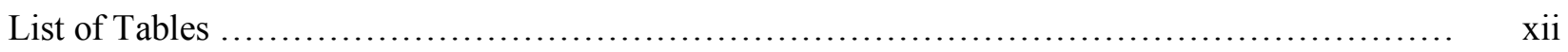

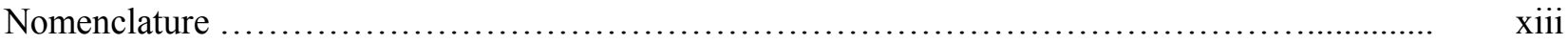

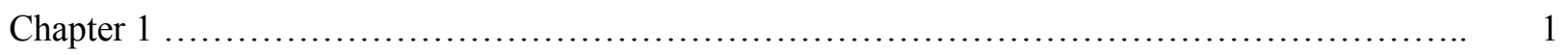

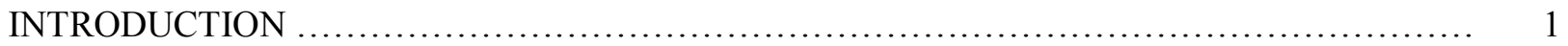

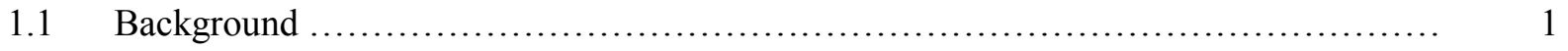

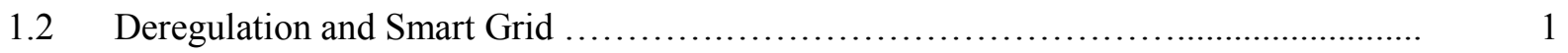

$1.3 \quad$ Distribution Systems ................................................................ 3

$1.4 \quad$ Distributed Generation............................................................... 5

1.4.1 Benefits of Distributed Generation ......................................... 5

1.4.2 Impacts of Distributed Generation........................................... 6

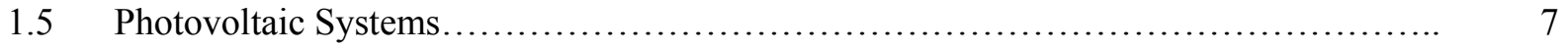

$1.6 \quad$ Distribution Power Flow...................................................................... 8

$1.7 \quad$ Price Based Unit Commitment...................................................... 9

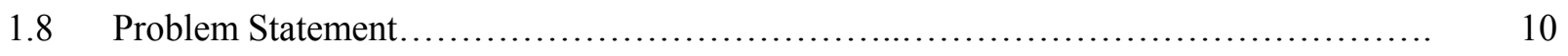

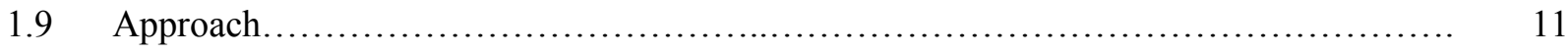


1.9.1 Distribution Feeder Model........................................... 11

1.9.2 Impacts of DG on Voltage Profile and Losses.............................. 11

1.9.3 Impacts of DG on Market Operations.................................. 12

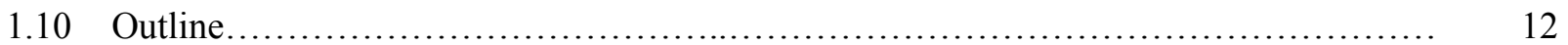

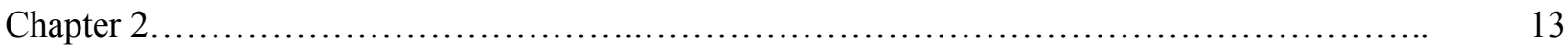

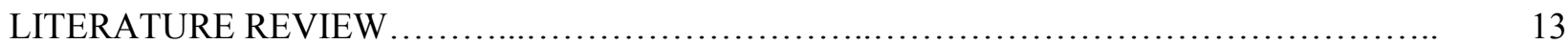

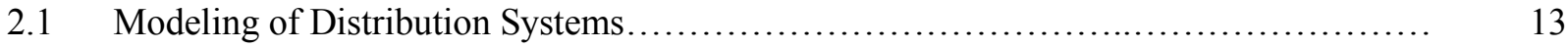

2.2 Distributed Generation in Distribution Systems............................... 14

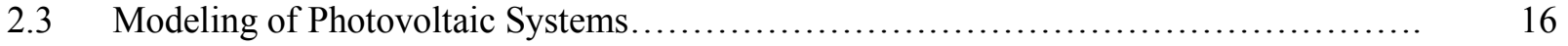

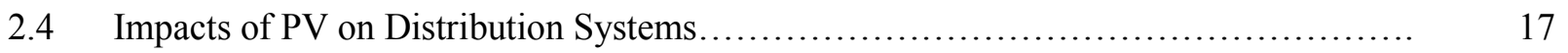

2.4.1 Impacts on Power Flow and Voltage Drop.............................. 18

2.4.2 Impacts on Reliability, Security and Network.......................... 18

$2.5 \quad$ Price Based Unit Commitment.................................................. 19

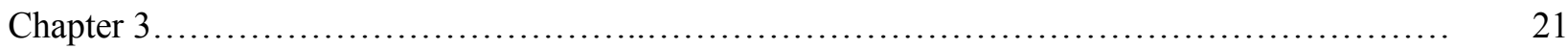

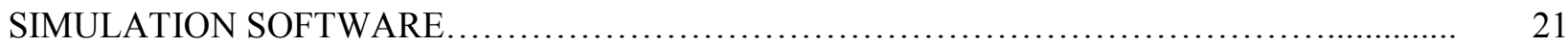

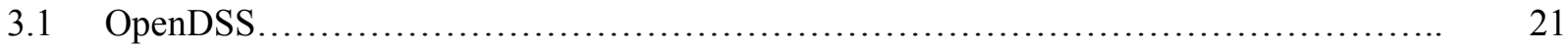

3.1.1 OpenDSS Architecture................................................ 22

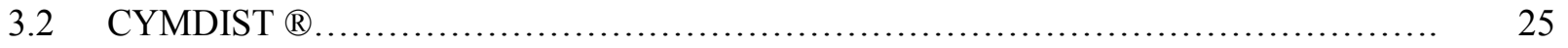

3.3 JAVA Programming..................................................... 27

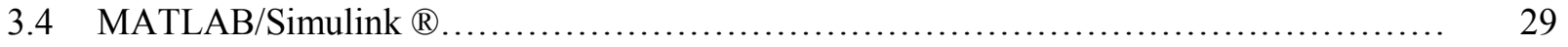

3.4.1 MATPOWER ..................................................... 30

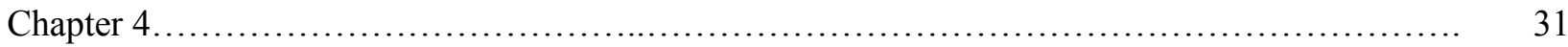




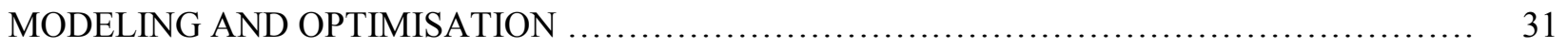

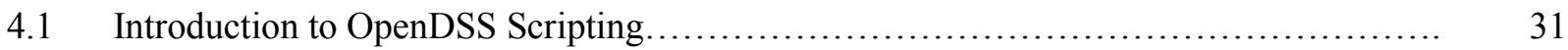

4.2 Geographical Location of Feeders........................................ 32

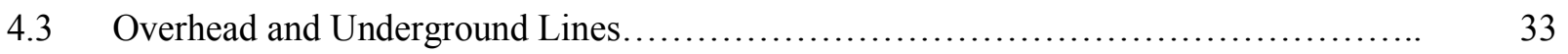

4.3.1 Line Geometry ................................................... 34

4.3.2 Line Code......................................................... 35

4.3.3 Wire Data......................................................... 36

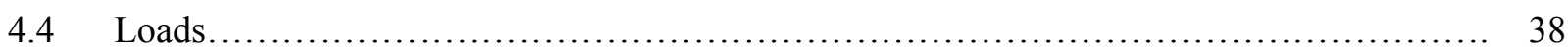

4.4.1 Distributed Load Model................................................ 39

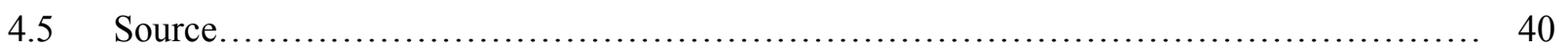

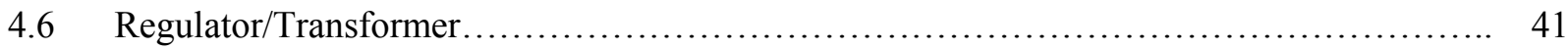

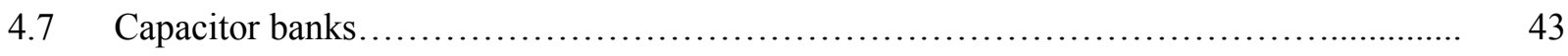

$4.8 \quad$ Switches, Breaker and Sectionalizer............................................ 45

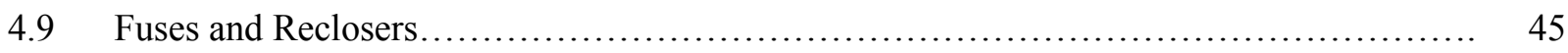

4.10 PV Model in Simulink......................................................... 46

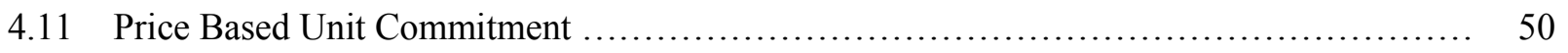

4.11.1 Nomenclature and Problem Formulation .................................. 50

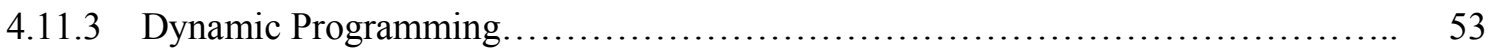

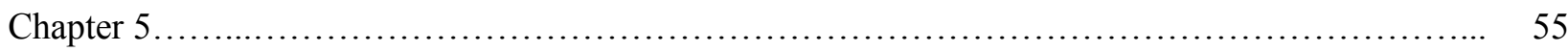

SIMULATION AND RESULTS...................................................... 55

5.1 Final Feeder Data Format in OpenDSS ....................................... 55

5.2 Distribution Power Flow Results for Feeder 1 of AEP system...................... 57 
5.3 Distribution Power Flow Results for Feeder 2 of AEP system...................... 58

$5.4 \quad$ Validation of Power Flow Results of AEP System................................. 59

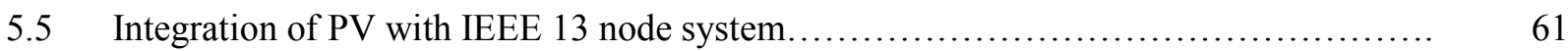

5.6 PV integration study on utility feeder model................................... 64

5.6.1 Voltage Profiles for Different Penetration Levels.......................... 66

5.6.2 Regulator Control Settings................................................ 67

5.6.3 Losses of the System.................................................. 69

5.6.4 Effect of Varying PV/Cloud Cover....................................... 70

$5.7 \quad$ Impacts of DG on power market operation..................................

5.7.1 Bidding Strategies with Wind Integration.............................. 74

5.7.2 Dispatch with forecasted wind power.................................. 76

5.7.3 Scenario I: Dispatch with low wind volatility $\ldots \ldots \ldots \ldots \ldots \ldots \ldots \ldots \ldots \ldots \ldots \ldots \ldots \ldots \ldots \ldots \ldots$

5.7.4 Scenario II: Dispatch with high wind volatility........................... 78

5.7.5 Scenario III: Dispatch with wind intermittency........................... 80

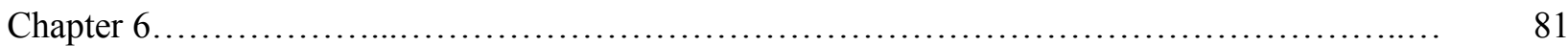

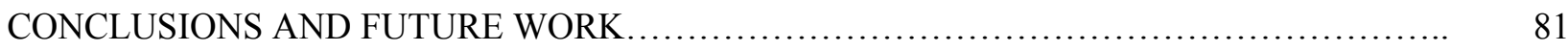

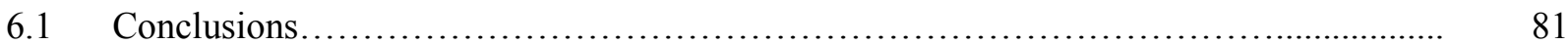

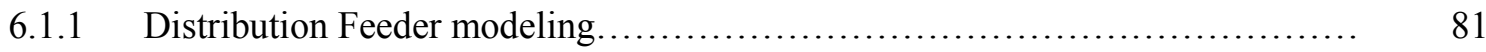

6.1.2 Integration of PV system to the Distribution grid.......................... 82

6.1.3 Impacts of DG on Market Operation..................................... 83

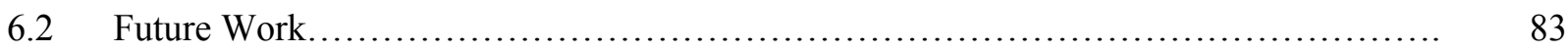

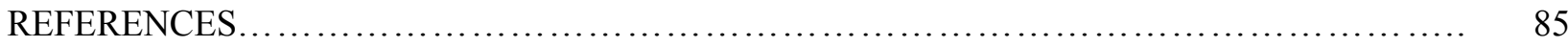




\section{LIST OF FIGURES}

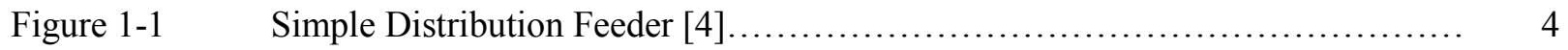

Figure $3-1 \quad$ Open DSS Architecture.......................................... 23

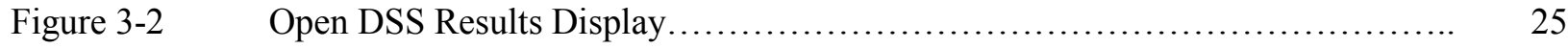

Figure $3-3 \quad$ CYMDIST Network Display........................................ 26

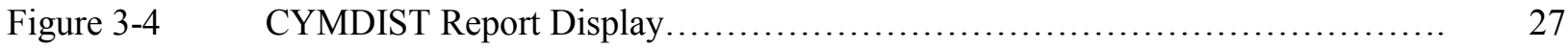

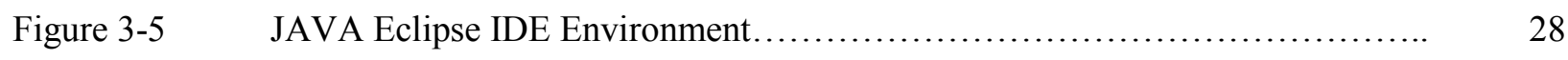

Figure 3-6 MATLAB Simulink model for PV system [90]........................... 29

Figure 4-1 Comparison of the CYMDIST and OpenDSS models for Bus coordinates...... 32

Figure 4-2 OpenDSS bus coordinates format and circuit plotting..................... 33

Figure 4-3(a) Comparison of the CYMDIST and OpenDSS models for overhead lines........ 33

Figure 4-3(b) Comparison of the CYMDIST and OpenDSS models for underground lines.... 34

Figure 4-4 Comparison of the CYMDIST and OpenDSS models for Loads............... 38

Figure 4-5 Distributed Load Model in OpenDSS ................................. 39

Figure 4-6 Comparison of the CYMDIST and OpenDSS models for Source.............. 40

Figure 4-7 Comparison of the CYMDIST and OpenDSS models for Regulator............ 42

Figure 4-8 Comparison of the CYMDIST and OpenDSS models for Capacitor............. 43

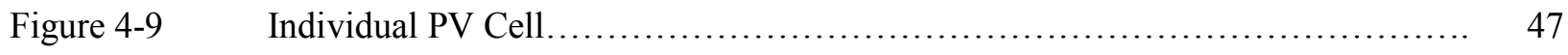

Figure $4-10 \quad$ Grid Connected PV system...................................... 47

Figure $4-11 \quad$ DC-DC Booster Model............................................ 48

Figure $4-12 \quad$ DC-AC Inverter Model............................................ 48 
Figure 4-13(a) V-I characteristics for insolation ranging from $0.25 \mathrm{~W} / \mathrm{m} 2$ to $1 \mathrm{~W} / \mathrm{m}^{2} \ldots \ldots \ldots . .49$

Figure 4-13(b) V-P characteristics for insolation ranging from $0.25 \mathrm{~W} / \mathrm{m} 2$ to $1 \mathrm{~W} / \mathrm{m}^{2} \ldots \ldots \ldots \ldots$

Figure 4-14 Utility scaled PV plant with interconnection transformer.................. 50

Figure 4-15 Stages in Dynamic Programming..................................... 54

Figure 5-1 Final Feeder Data Model Conversion in OpenDSS...................... 56

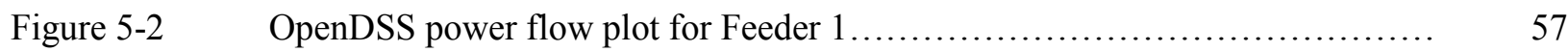

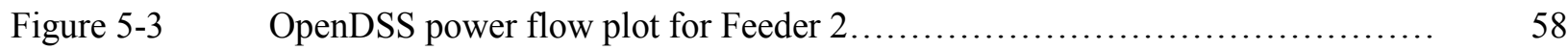

Figure 5-4(a) Section Currents in CYMDIST and OpenDSS........................... 60

Figure 5-4(b) Node Voltages in CYMDIST and OpenDSS .............................. 60

Figure 5-5(a) Real powers through a section in CYMDIST and OpenDSS $\ldots \ldots \ldots \ldots \ldots \ldots \ldots . \ldots 1$

Figure 5-5(b) Reactive powers through a section in CYMDIST and OpenDSS.............. 61

Figure 5-6 IEEE 13 Node test feeder with PV at end node......................... 62

Figure 5-7 Phase Voltage variations of IEEE 13 node feeder with PV................... 63

Figure 5-8 AEP Feeder model with PV and meter locations......................... 64

Figure 5-9 Time Varying PV and load profiles.................................... 65

Figure 5-10(a) Base case Voltage profile of the circuit with controls....................... 66

Figure 5-10(b) Voltage profile of the circuit with controls with 10\% PV penetration............. 66

Figure 5-10(c) Voltage profile of the circuit with controls with 30\% PV penetration............ 66

Figure 5-10(d) Voltage profile of the circuit with controls with 50\% PV penetration............ 66

Figure 5-11(a) Source node voltages - Existing control settings........................... 68

Figure 5-11(b) Source node voltages with lower voltage regulator set point.................. 68

Figure 5-11(c) End node voltages- Existing control settings............................ 68 
Figure 5-11(d) End node voltages with lower voltage regulator set point................... 68

Figure 5-12 24-hour Three phase Regulator tap changes............................. 69

Figure 5-13 Percentage System losses for various penetration levels................... 70

Figure 5-14 10-minute varying PV and load shapes to simulate cloud cover............... 70

Figure 5-15 Source node voltages with 50\% PV penetration under a cloud cover scenario... 71

Figure 5-16 End node voltages with 50\% PV penetration under a cloud cover scenario..... 71

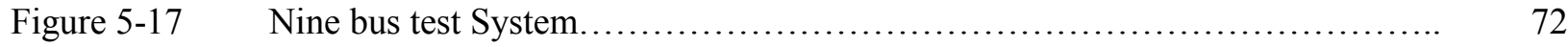

Figure $5-18 \quad$ Forecasted Wind farm output...................................... 74

Figure 5-19 Variation of Market Clearing Price with Wind Integration.................... 75

Figure 5-20 Bidding Strategy of the GENCO with and without wind.................... 77

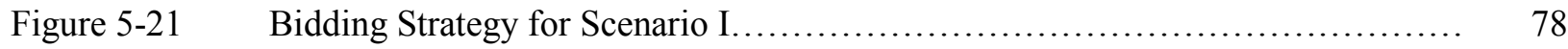

Figure 5-22 Bidding Strategy for Scenario II.................................... 79

Figure 5-23 Bidding Strategy for Scenario III...................................... 80 


\section{LIST OF TABLES}

Table 1-1: $\quad$ Electrical Characteristics of Distribution System Components................. 5

Table 4-1: $\quad$ Line Geometry Properties.......................................... 35

Table 4-2: $\quad$ Line Code Properties............................................... 36

Table 4-3: Conductor Properties.......................................... 37

Table 4-4: $\quad$ Load Properties................................................... 39

Table 4-5: Voltage Source Properties....................................... 41

Table 4-6: $\quad$ Regulator Properties............................................. 42

Table 4-7: $\quad$ Capacitor Properties................................................ 44

Table 4-8: $\quad$ Fuse/Recloser Properties......................................... 45

Table 4-9: $\quad$ Typical Data Sheet Parameters........................................ 49

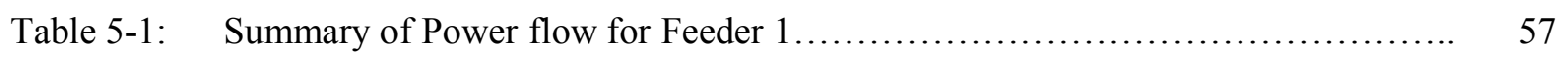

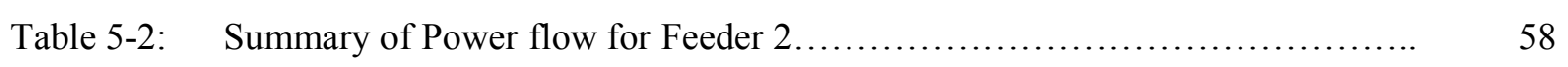

Table 5-3: Maximum and average error for conversion from CYMDIST to OpenDSS..... 59

Table 5-4: Voltage results of IEEE 13 node feeder with $10 \%$ PV penetration............... 62

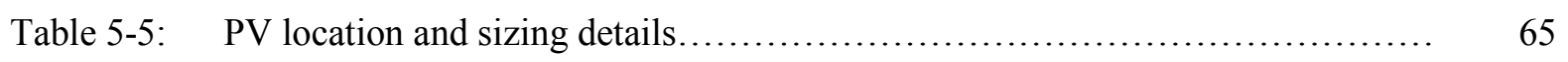

Table 5-6: $\quad$ System Bus/Branch Data............................................ 73

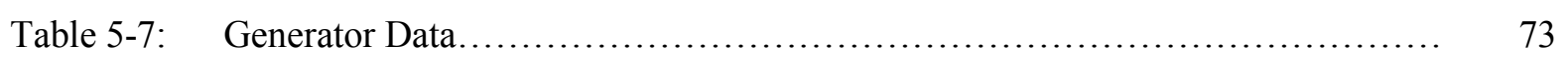

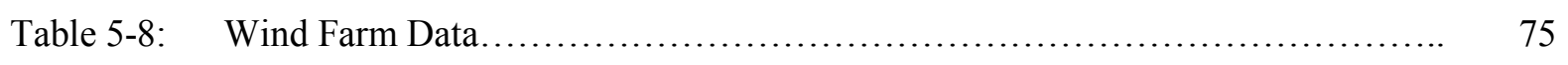

Table 5-9: $\quad$ PBUC Plans for the Generators..................................... 77 


\section{NOMENCLATURE}

\begin{tabular}{|c|c|}
\hline FERC & Federal Energy Regulatory Commission \\
\hline ISO & Independent System Operator \\
\hline RTO & Regional Transmission Organization \\
\hline AEP & American Electric Power \\
\hline DG & Distributed Generation \\
\hline LTC & Load Tap Changer \\
\hline CHP & Combined Heat and Power \\
\hline PX & Power Exchange \\
\hline MCP & Market Clearing Price \\
\hline GENCO & Generation Company \\
\hline DISCO & Distribution Company \\
\hline PBUC & Price Based Unit Commitment \\
\hline PV & Photo Voltaic \\
\hline CSV & Comma Separated Values \\
\hline XML & Extended Markup Language \\
\hline DSS & Distribution System Simulator \\
\hline DMS & Distribution Management System \\
\hline EMS & Energy Management System \\
\hline LR & Lagrangian Relaxation \\
\hline
\end{tabular}




\section{Chapter 1}

\section{Introduction}

\subsection{Background}

The electric power grid is one of the most complex and costly investments made by mankind all over the world. An electrical grid is a vast, interconnected network for delivering electricity from suppliers to consumers of electric power. The modern day electric industry is poised to make the transformation from a centralized, producer-controlled network to one that is more distributed and consumer-interactive. This deregulated model creates healthy competition among the market forces and reduces the cost of electricity delivered. The move to a -marter" grid promises to change the industry's business model and its relationship with all stakeholders, involving utilities, regulators, energy service providers, technology and automation vendors and all the consumers. This transformation is made possible by bringing the philosophies, concepts and technologies from research labs to the utility and the electric grid.

\subsection{Deregulation and Smart Grid}

Before deregulation, electric utilities took a vertically integrated approach to their power system, which means a single company provided generation, transmission and distribution to customers. In a deregulated market, all these functions have been separated, as mandated by the Federal Energy Regulatory Commission (FERC) under Order Number 2000 which endorses competitive power markets and price signals for the purpose of managing electricity grid congestion and achieving reliability [1]. An Independent System Operator (ISO) or Regional Transmission Organization (RTO) is a profit neutral organization in charge of managing supply and demand as it coordinates controls and monitors the operation of the power system. The ISO and RTO were formed at the recommendation of the FERC and their role is significant in bringing the Smart Grid concept to reality. In the Smart Grid environment, ISOs and RTOs use smart distribution system concepts for managing a secure and economic power system. The aim of ISOs and RTOs is to create a more - marter" and modern electrical grid, which is needed to meet the demands of the $21^{\text {st }}$ century. The US Department of Energy defines the Smart Grid as having the following principal characteristics [2]. 
1) Self-healing: Continuous self assessment of the modern grid would detect, analyze, and respond to contingencies and restore faulted sections of the network.

2) Motivation and Inclusion of Customer: There would be an increased interaction with the consumer, by providing real time pricing and demand response options which reduce the cost of electricity delivered.

3) Resist attack: The modern grid has to withstand physical or cyber attacks and improve public safety.

4) Provides power quality for $21^{\text {st }}$ century needs: Improved power quality standards leads to better service and avoids productivity losses.

5) Accommodates all generation and storage options: Diverse natural and renewable energy sources that can be used in a -plg and play" mode provide cheaper and cleaner generation.

6) Optimizes assets and operates efficiently: Minimum cost operational principle guides the operations and leads to fuller utilization of assets. More maintenance programs results in fewer equipment failures.

7) Enables markets: The open market system reduces wastage and inefficiency and offers new choices to the consumer.

There are enormous advantages to the nation with the inception of Smart Grid [3]. The chances of cascading outages, like the North East black out of 2003 are greatly reduced. A smarter grid can reduce the country's dependence on foreign fuel and improve the overall security of the grid through deterrence of any physical or cyber attacks. An important goal of Smart grid is to accommodate all generation and storage options. With increasing penetration of Distributed Generation (DG) and introduction of new storage technologies, the energy losses are reduced and energy is generated with a reduced environmental impact. DGs are small scale power generation technologies located close to the load being served, capable of lowering costs, improving reliability, reducing emissions and expanding energy options. The main advantage with the use of DG is the improvement in system adequacy and security. Overall, there is a societal benefit that includes greater US competitiveness, job creation and customer service enhancements. 


\subsection{Distribution Systems}

The distribution system typically starts at the distribution substation, and is fed by one or more subtransmission lines. Each substation is designed to serve one or more primary feeders. Most of the utility distribution feeders are radial, i.e. power flows from the substation to the metered user. A layout of a simple distribution system and its components is shown in Figure 1-1. An important characteristic of radial distribution feeders is having only one path for power to flow from the source to each customer. A typical distribution system is composed of distribution substations having one or more feeders. Each feeder consists of three phase primary main feeder with branching laterals that are two phased or single phased. The distribution lines may be overhead or underground depending on the feasibility and requirement, with distinct electrical characteristics. Voltage regulators adjust the voltage settings, to keep the voltage at all nodes within ANSI limits. Some of the primary main feeders have in-line transformers to serve large industrial consumers. To provide reactive power support to the feeder at critical nodes, single phase or three phase capacitor banks are used. The substation transformer primary usually operates at $12.47 \mathrm{kV}$ and the voltage is stepped down to $4.16 \mathrm{kV}$. Smaller distribution transformers, also known as service transformers supply customers at $120 / 240 \mathrm{~V}$ level. The distribution feeder supplies single phase, two phase and three phase loads categorized as smaller residential consumer as well as large industrial consumers. The important characteristics of distribution systems are highlighted below [4].

- A distribution substation reduces the supply voltage to distribution system level utilizing the substation transformer. The standard distribution voltage levels are $34.5 \mathrm{kV}, 23.9 \mathrm{kV}, 14.4 \mathrm{kV}, 13.2$ $\mathrm{kV}, 12.47 \mathrm{kV}$, and in older systems, $4.16 \mathrm{kV}$.

- There is a voltage drop from the substation to the load end of the feeder. This voltage drop largely depends on the loading conditions of the feeder. The distribution substation regulates the system voltage within acceptable ANSI limits using the Load Tap Changing Transformer (LTC).The LTC adjusts the taps on the secondary side of the substation transformer as the load varies and adjusts the voltages to be within limits.

- The distribution system consists of simple switches and high voltage circuit breakers for high voltage switching and relay controlled circuit breakers to perform low voltage switching. In certain cases reclosers are used instead of the relay circuit breaker combination. 


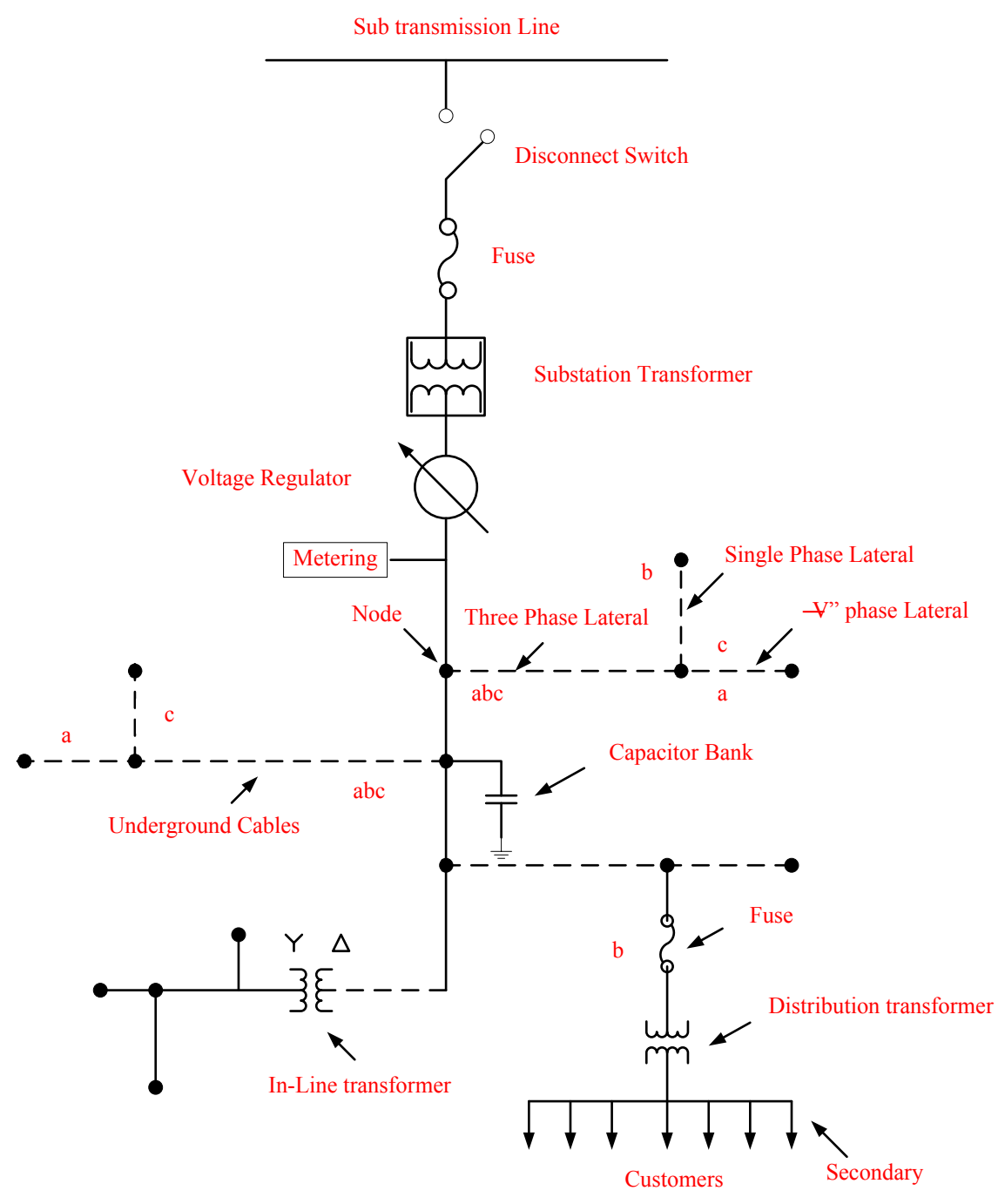

Figure 1-1 Simple Distribution Feeder [4]

Apart from these features, each device in a distribution feeder has unique electrical characteristics that must be determined before the power flow analysis of the feeder. The important electrical characteristics of each component are shown in Table 1-1. 
Table 1-1 Electrical Characteristics of Distribution System Components

Overhead
Conductors
- Spacing
- Phasing
- Distance
from ground
level
-Wire details
-Kron
reduction

\begin{tabular}{l} 
Underground \\
Conductors \\
- Spacing \\
- Phasing \\
- Thickness of \\
tape shield \\
- Number of \\
Concentric \\
Neutrals \\
-Cable details \\
\hline
\end{tabular}

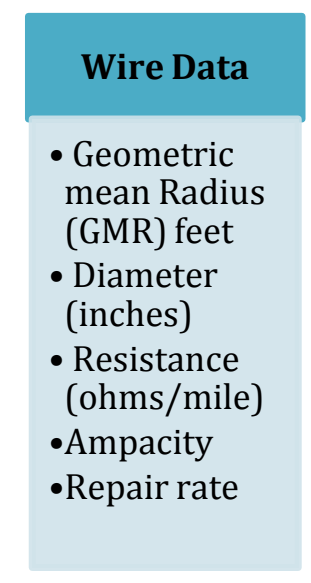

\begin{tabular}{l} 
Voltage \\
Regulators \\
- Potential \\
Transformer \\
ratios \\
- Current \\
transformer \\
ratios \\
-Compensator \\
settings \\
- R and X \\
settings \\
\hline
\end{tabular}

Transformers
- kVA rating
- Voltage rating
- Impedance
settings
(R and X)
- No-load
power loss

Capacitors

- Capacity

- Phasing

- Control type

- ON-OFF settings

-Power factor

\subsection{Distributed generation}

With the deregulation of the electric power industry and the advancement of new technologies, the attention of the utilities has been drawn towards adapting Distributed Generation (DG) into their existing infrastructure. The deployment of DG does bring ample technological and environmental benefits to the traditional distribution networks [5]. The appropriate sizing and placement of DG systems which generate power locally to fulfill consumer demands, helps to reduce power losses and avoid transmission and distribution system expansion. In the last few years, renewable energy has gained ground as a viable future source of energy in the electric industry. The most popular forms of DG are wind power, solar photovoltaics, fuel cells, micro-turbines and small diesel generators. DGs can be classified as renewable and non-renewable energy sources based on the use of primary energy. Some DGs that are of electromechanical type could be directly interfaced to the power system whereas some other DGs require inverter based systems to connect to the grid. The ample benefits of DG along with their numerous impacts are discussed in the following sections.

\subsubsection{Benefits of Distributed Generation}

- More environmentally friendly: The modern grid encourages the deployment of smaller DG sources based on clean technology and those employing highly efficient Combined Heat and Power (CHP) technology. Because of the greater efficiency of CHP, carbon emissions per unit of useful energy are substantially lower than those of conventional fossil fuel based plants. Some of the popular forms of DG are wind energy and solar energy which are environmentally friendly and produce zero emissions. These zero emission sources have greater access to the grid with their increasing penetrations. These 
renewable sources currently represent about $2 \%$ of US generation capacity. Various organizations have recommended renewable penetration to be raised above $10 \%$, resulting in a significant reduction of green house gas emissions.

- Reduce the need for new transmission lines: The DGs reduce the need for new centralized generating stations and associated transmission lines. The proper application of FACTS devices to DGs will allow the deferral of some transmission line additions.

- Promote demand response and energy storage: DGs are renewable sources of energy and when coupled with demand response and energy storage, they can be more viable and can contribute to a greater percentage of supply.

- Enable Renewable Portfolio Standards: Introduction of low emission DGs enables many states to meet their goals for Renewable Portfolio Standards.

- Reduce Electrical Losses: The modern grid is made more efficient by accommodating many generation alternatives. Generation sources, including plants, located near load centers reduce transmission losses [6]. The reduction of harmonics and momentary voltage excursions due to DGs will reduce electrical losses.

\subsubsection{Impacts of Distributed Generation}

- Impact on Power System planning: One of the main tasks of distribution network planning is to provide reliable and high quality power supply based on generation and load forecasting. The DG reduces the dependence of the distribution network on large scale power plants and transmission lines. The random input and withdrawal of DG sources from the grid increases the uncertainty of power system load forecast. This randomness leads to an urgent need to create a reasonable distribution system network by effectively coordinating the various DG sources.

- Impact on Power flow and losses: Power flow in traditional radial distribution networks is unidirectional. The introduction of DG into the distribution network causes two way power flows and complex voltage changes [7]. The losses of the grid largely depend on the power flow. DG may increase or reduce losses, depending on the location, capacity of DG and the relative amount of loading quantity, as well as the network topology and other factors.

- Impacts on short circuit currents and relays: The existing relay and protection settings of a distribution grid are based on unidirectional power flow. With the power injection from DG sources, the direction and duration of the short circuit currents change. The operation of the DG may lead to 
failure or wrong operation of the relays, and this result in changes in the fault levels of the distribution network.

- Impact on voltage profile: DG causes a significant change in the voltage profile of the feeder, depending on its location and size. The traditional regulator settings and placement of capacitor banks need redesign according to the new voltage profiles.

- Impact on power quality: DG devices need power electronic converters for efficient connection to the grid. A large number of power electronic inverters in the grid causes the distortion of the current and voltage waveforms and introduce harmonics. DGs produce voltage flicker and harmonics in the circuit [8]. Also, the start up and the drastic changes in the DG power output cause flicker that adversely affects power quality.

- Impact on power market: The introduction of the DG sources creates a new market structure with different choices of suppliers, periods of electricity, quality of power, and rate structures [9]. The DG sources that depend on natural factors like wind and sunlight, can impact the power market operation under conditions of volatility and intermittency. The availability of winds and sudden cloud covers can affect wind and solar DG operation. Under such circumstances, the utility must accommodate additional reserves to meet the demand.

\subsection{Photovoltaic Systems}

Solar energy is the world's major renewable energy source and is available everywhere in different quantities. Solar photovoltaics are one of the fastest growing DG technologies, with a lot of utilities in North America expressing interest in its implementation [10]. Their annual growth rate in the power market is estimated to be about $25-35 \%$. Until a few years back, solar energy was mainly used for off grid applications like rural electrification, water pumping and heating while most of the newly installed PV is used directly in the distribution grid. The largest solar power installation in the world is the Solar Energy Generating Systems facility in California, which has a total capacity of 354 Megawatts (MW). Nevada Solar One is a solar thermal plant with a 64 MW generating capacity, located near Boulder City, Nevada. Many utilities either receive incentives or are mandated by green-generation portfolio regulations to install solar PV systems on their feeders. Photo-voltaic systems have become increasingly popular and are ideally suited for distribution systems. Because of strict environmental regulations, the lack of corridors for building high voltage transmission systems and security issues, larger power plants have become uneconomical in many regions. Additionally, recent technological advances in small generators, power electronics, and energy storage devices have provided a new opportunity for PV systems that are located 
closer to loads. In spite of their relatively high cost, there has been remarkable growth in installed photovoltaic systems. There is ongoing research aimed at reducing the cost and achieving higher efficiency [11]. Currently, photovoltaic generation systems are actively being promoted in order to mitigate environmental issues such as the green house effect and air pollution. Photovoltaic panels do not have any moving parts, operate silently and generate no emissions. Another advantage is that solar technology is highly modular and can be easily scaled to provide the required power for different loads.

Grid connected PV systems are typically MW size plants (e.g. 1-10 MW) connected to conventional feeders or dedicated express feeders. The MW size PV systems along with the power electronic inverter are connected in parallel to the grid and sometimes the load is served only when the grid is available. The energy produced by the PV system decreases the apparent load, excess energy flows into the grid. Utility scale solar farms usually have nominal capacities compatible to medium voltage feeders and require transformers at the point of common coupling. The PV system usually produces power at unity power factor and the utility must undertake all the VAR requirements. IEEE 1547 standards on DG interconnection with the grid require that there must be no direct communication or control between the inverter and the utility [12]. Hence when the grid voltage/frequency deviates from the boundaries, the inverter must disconnect itself from the grid until normal conditions resume. Almost all the inverter modules are equipped with overcurrent and under/over voltage protection schemes to prevent islanding and thus restrict the PV plant from feeding power to the grid in the event of a fault. Geographical factors influence the efficiency of grid connected PV systems by restricting the PV production to coincide with the times when it is most economical for the utilities to use. A desirable feature of the PV systems is the design of efficient storage so that the system can operate independent of the grid.

\subsection{Distribution Power Flow Algorithm}

The distribution system has to serve a large number of unequal single phase loads; hence the loading of the system becomes inherently unbalanced. An additional unbalance is introduced by the non equilateral conductor spacing of three-phase overhead and underground line segments. The lines in a distribution network have higher $(\mathrm{R} / \mathrm{X})$ ratios. This makes the distribution system ill-conditioned and hence the fast decoupled Newton method is not suitable for power flow solutions. Due to the unique radial of the distribution system, conventional power-flow and short-circuit programs used for transmission system studies are not adequate and display poor convergence characteristics for radial distribution systems. The constant power demand assumption does not hold good for a distribution system 
because the system bus voltages are not constant. Hence the load flow algorithm becomes non-linear and iterative methods must be employed for solving them [13]. A specialized iterative technique called the forward backward sweep is used for distribution system load flow and short circuit analysis. It is imperative that the distribution feeder is modeled accurately to perform accurate power-flow and shortcircuit studies by utilizing the three-phase models of the major components.

There are some challenges in modeling each feeder accurately. One of the most difficult tasks is to acquire all the necessary data. Feeder maps contain most of the needed data. Additional data such as standard pole configurations, specific conductors used on each line segment, three-phase transformer connections, and voltage regulator settings comes from the utility stored records. The loads on the distribution system are not constant and the metering is prone to errors, hence an accurate representation of the load is not possible. The impedance calculations of three phase unbalanced overhead and underground lines require accurate models. In this thesis a comprehensive modeling study is done for a utility distribution feeder and some of these modeling challenges are considered.

\subsection{Price Based Unit Commitment}

The reliable and efficient operation of a deregulated market structure is ensured by an independent body known as the ISO. The ISO establishes rules for energy and ancillary services markets, manages the system in a fair and non- discriminatory manner and shields the markets from risks and accumulation of market power with a single entity. In order to achieve these goals, the ISO supports different market models namely the PoolCo, Bilateral Contracts and Hybrid models. The PoolCo market model is defined as a centralized marketplace that clears the market for power buyers and sellers. Electric power sellers/buyers submit bids to the pool and each bid contains information on how much power, at which prices, in which area, at what time, a market participant is willing to buy or sell. The PoolCo market model is achieved by the Power Exchange (PX) that is integral to the ISO's operation. The PX functions as an independent, non-government and non-profit entity that conducts the auction for electricity trades in the market. The PX calculates the market-clearing price (MCP) based on the highest price bid in the market.

In such a competitive market, Genco (Generation Company) sells electricity to the PX from which large customers such as DISCOs (Distribution Company) and aggregators may purchase electricity to meet their needs. Along with real power, Gencos indulge in trading of reactive power and operating reserves. For successful bidding in the market, Gencos need innovative strategies to determine their optimal bid to maximize revenue and profit targets. Generation schedules covering a range of 24 hours to 
1 week ahead achieved through unit commitment, serve this purpose for a competitive Genco.

In the deregulated power market, a particular type of unit commitment is used by the Genco to optimize generation resources in order to maximize its profit, called the Price Based Unit Commitment (PBUC) [14]. In PBUC, satisfying load is no longer an obligation and the objective is of maximizing the profit from trading energy and Ancillary Services (AS) in the market. The distinct feature of PBUC is that the market price reflects on all market transactions indicating market price as the only signal that enforces a unit's ON/OFF status and generation dispatch. In day-ahead market Genco runs PBUC based on forecasted energy and ancillary services price, and price uncertainty needs to be considered as it has direct impact on the expected profit. PBUC is a combinatorial optimization problem and a Lagrangian relaxation technique coupled with dynamic programming is used in this thesis to determine the PBUC schedules of the Genco.

Apart from innovative bidding strategies, Gencos have adopted DG resources such as wind farms to their portfolio; to supplement coal/natural gas fired generation and meet green generation mandates thereby maximizing profits. For a Genco with a wind generation, wind power availability forecast is very essential as it has a direct impact on the system performance and stability. With the availability of wind, a suitable adjustment must be made in its pricing, and this is usually related to the uncertainty in wind power availability. This thesis addresses the effects of availability of the DG resource on the PBUC schedules of the Genco by integrating the effects of wind intermittency.

\subsection{Problem Statement}

The primary objective of this thesis is to model AEP distribution feeders in OpenDSS. This thesis develops models for $12.47 \mathrm{kV}$ (medium voltage) distribution feeders in OpenDSS by utilizing the existing models in CYMDIST. The model conversion is achieved by a detailed one-to-one component matching approach for multi phased lines, conductors, underground cables, loads, regulators and capacitor banks. The power flow results of OpenDSS and CYMDIST are compared to derive important conclusions.

The second major objective is to analyze the impacts of DG on distribution systems and two focus areas are chosen, namely: effect on voltage profiles and losses of the system and the effects on power market operation. To analyze the impacts of DG on the distribution systems, Photovoltaic (PV) system with varying penetration levels are integrated at different locations along the developed feeder model. The effects of voltage unbalance and the losses of the feeder are analyzed for different PV location and penetration scenarios.

This research work assesses the impact of DG on power market operations. Under a deregulated 
market structure, a particular type of unit commitment is used by the Genco to optimize generation resources in order to maximize its profit, called the Price Based Unit Commitment (PBUC). These innovative profits based bidding strategies change when Gencos adopt DGs such as wind farms to their portfolio. The inclusion of intermittent DG resources such as wind farms by the Gencos causes uncertainty in PBUC schedules. The effects of intermittency in the DG resource availability on the PBUC schedule of a Genco owning a distribution side wind farm are analyzed for different levels of wind volatility.

\subsection{Approach}

The sections to follow will explain the complete modeling strategies. The key aspects of this entire work are highlighted below in simple terms.

\subsubsection{Distribution Feeder Model}

- The CYMDIST data obtained from the utility is available in two different formats, namely the XML format and CSV format.

- The XML format represents the system data in between a starting and an ending tag. The CSV format provided system data in a text file, with the system data as comma separated values. The CYMDIST model contained network information providing the details regarding the location of the feeders and its components and load information providing the load details for the system for all nodes.

- A JAVA program is used represent the CYMDIST data in OpenDSS, by a one-on-one parameter mapping. The converted DSS data are organized according to OpenDSS file structure.

- $\quad$ Power flows are run on both the feeders and results are compared to CYMDIST model.

\subsubsection{Impacts of DG on Voltage Profile and Losses}

- The developed feeder model is used to assess the impacts of PV penetration and location.

- The load shapes for 24 hour time varying load and PV insolation are considered for a three phase unbalanced distribution power flow.

- The node voltages from the source to the end of the feeder are analyzed using energy meters at strategic locations.

- The losses for the feeder over a 24-hour period and section wise losses are also analyzed. 


\subsubsection{Impacts of DG on Market Operations}

- A deregulated market scenario is considered for analyzing the PBUC strategy of a Genco owning a distribution side wind farm.

- The PBUC schedules for the Genco, with and without the inclusion of the wind farm are developed using Lagrangian relaxation technique with Dynamic Programming, and the market clearing price variations are taken into account.

- The schedules in real time market, energy and ancillary services dispatch to fulfill contractual obligations are evaluated for low and high wind volatility and brief intermittency.

\subsection{Outline}

The rest of this thesis document is organized as follows.

Chapter 2 presents a literature review on modeling of distribution systems. The impacts of distribution systems on the distribution systems operation and the impacts on power markets are also discussed.

Chapter 3 gives a detailed description of the software packages used in this. The applications of these software and their salient features are highlighted.

A detailed and comprehensive description of the utility distribution feeder model is given in Chapter 4. The modeling of various components like multi phased lines, cables, loads regulators, capacitor banks and the utility feeder model for three phase unbalanced distribution power flow is presented. Photovoltaic system model used in this study and its details are given. The PBUC optimization model for a Genco owning a distribution side wind generator is presented.

Load flow results for the converted feeder model in OpenDSS and comparison with CYMDIST is presented in Chapter 5. Different scenarios pertaining to PV location and penetration along the feeder and 24-hour power flow and voltage analysis results for the same are also presented. The PBUC schedules for a Genco, for different scenarios of wind intermittency are developed.

Finally, conclusion of this study and the future work is detailed in Chapter 6. 


\section{Chapter 2}

\section{Literature Review}

A detailed literature survey covering all aspects of this thesis is presented in the following sections.

\subsection{Modeling of Distribution Systems}

There has been a considerable amount of work done with regards to the modeling of distribution systems. Some of the important aspects related to the modeling and analysis of distribution systems are presented. The models and algorithms developed over the years to analyze electrical circuits were restricted to transmission networks and were called Energy Management Systems (EMS). The application of these algorithms to distribution systems resulted in reduced efficiency of calculations and lack of convergence of solutions. In recent years specialized algorithms and models for distribution networks have been developed and are called as Distribution Management Systems (DMS). Reference [15] provides an overview of the DMS. The specific features of distribution systems like radial nature, large dimension of the system, high value of $\mathrm{R} / \mathrm{X}$ ratios and reduced measurements are addressed by DMS. A network model is the core of DMS and other functionalities are based upon the network model. The network model consists of single phase and multi phase representation of the network with details of different components like lines, cables, transformers and regulators. In [16], a method of experimental design is used to verify the qualitative model of the DMS and analyze the significance of each component. Results presented in [16] demonstrate that the computation of impedances and the evaluation of active loads at the system buses have a fundamental effect on the accuracy of the computations.

The distribution power flow is one of the most important functions of a DMS. As a distribution system is vast, the errors in the power flow solutions are influenced by a large number of parameters. Ladder method (backward/forward sweep), the power summation method and the current summation method are commonly used to obtain power flow solutions for distribution systems. A comparative study of these algorithms is presented in [17]. The results of the studies indicated that at increased system load levels, the power summation method performed better. The ladder method has limitation on depth of system sub-feeder as each sub feeder analysis requires separate iterations. For nominally loaded systems 
the current summation method fares as well as the power summation method. Moreover, the power flow results of a test system with balanced, decoupled models are compared with unbalanced, multi phase models [18] to show significant differences. It is seen that the error of approximating a balanced system increases with the loading level. Hence it is important to use an unbalanced, multi phase modeling for operational applications such as volt/var control and service restoration. Many research works have considered the improvement of individual component models for better power flow solutions. It is a common practice to define loads as constant current, constant impedance and constant power. While this definition may be sufficient for a single snapshot power flow, detailed load behavior is necessary for time series simulations. An integrated load model is presented in [19] that can be used for time series power flows. Similarly, the regulators can be defined as either line drop compensators or as transformers with very low impedance and losses. The impedance of the transformers in the model has been shown to reflect the losses in the system [20]. The authors present a detailed model of the On-Load tap changing transformer for distribution systems to improve the loss calculations.

Reference [21], a program concept paper developed by EPRI, is of particular interest as it provides an overview of the modeling of two North American feeders by converting their study from a commercial analysis tool to OpenDSS. The aim of this paper was to represent the two feeders in the Common Information Model (CIM) format as a benchmark for interoperable studies. A detailed gap analysis in the modeling of commercial tools like CYMDIST, AREVA DMS, ABB and the open source OpenDSS is provided. Some of the modeling challenges provide a good insight into the issues facing the interoperable studies for distribution system analysis tools. Reference [22] provides a common data model for electrical distribution systems and the requirements for modeling lines, loads, transformers and specific distribution devices. The work on an IEEE benchmark feeder provided a set of modeling guidelines based on CIM standards. Reference [23] gives the latest updates on the IEEE Distribution System Analysis Subcommittee's efforts for CIM in distribution systems. Details on the interoperability tests conducted for standardization studies and the consistency of CIM models for transmission and distribution are also provided.

\subsection{Distributed Generation in Distribution Systems}

With the wide acceptance of DGs, traditional power system planning and operation face substantial challenges. The accurate modeling and analysis of DG sources is necessary for power flow calculations. Reference [24] provides an overview of the modeling guidelines for DG systems. The synchronous machine models for rotating type systems and the power electronic models for static devices like PV are 
highlighted. The important parameters for modeling these systems include the apparent power, RMS phase-to-phase voltage, frequency and duty cycle. The voltage regulator control and capacitor control are also provided for the distribution system with DG. In [25], an unbalanced load flow algorithm with DGs is described. The authors have considered the operation of DG in two different modes namely constant voltage and constant power factor. A comprehensive study of DG penetration on IEEE bench mark feeders shows losses and voltage deviations for varying penetration levels. The results are compared with commercially available tools to demonstrate the accuracy of the algorithm for the various test cases. Reference [26] provides detailed modeling of DGs and their integration into the distribution network using an efficient compensation based load flow technique. The authors conclude that photovoltaic systems and fuel cells can be considered as PV buses and wind turbine generators can be represented as PV/PQ buses. Another conclusion of this study is that the compensation based load flow technique is very robust for the radial systems with DG. In [27] the impact of the various models of the DG on the performance of the backward forward sweep algorithm is discussed. The authors proved that various DGs modeled as PV nodes perform well with backward forward sweep algorithm for weakly meshed networks. In [28], a new three phase backward forward sweep power flow for the weakly meshed distribution network with DG is presented. The authors use a compensation technique to handle PV nodes in a weakly coupled network. References [29-30] present a Zbus based method to overcome the shortcomings of handling PV nodes effectively. The traditional Zbus method is modified, and a new matrix, based on the sensitivities of the distribution lines, which adjusts the real and reactive power mismatches, is included in the power flow.

DGs can act as energy sources as well as static var compensators. Suitable control algorithms must be designed to regulate the power output of DG to prevent voltage unbalance and harmonic currents. Many research works discuss the various control strategies for regulating the flow of power from the DG to the network. In [31] authors propose a decoupled real and reactive power control approach to control the power flow of DG. By adjusting the power angle, the real power is controlled and by adjusting the filter capacitor voltage, the reactive control is achieved. Simulation results have demonstrated the fast response of the decoupled control algorithm. In [32] a feedback loop control design is proposed to regulate the power flow of the DG and balance the voltage along the feeder. The simple control loop using local voltage information minimizes the voltage unbalance and the line losses. Reference [33] proposes a similar control strategy based on the feed forward algorithm using phase locked loop algorithm. The effectiveness of the proposed scheme in handling harmonic currents and distorted line voltages are demonstrated by simulation results. 


\subsection{Modeling of Photovoltaic Systems}

Many utilities, over the years have been undertaking studies to examine and quantify the potential benefits of distributed grid-connected Photovoltaic (PV) generation [34-35]. It is found that PV generation is potentially cost effective alternative for utility planning in terms of enhanced reliability, system expansion, environmental mitigation and losses of the system. The studies concluded that the most important electrical impacts of PV generation were relieving thermal overloads, electrical loss savings, kVAR support the system capacity value.

Most of the research work is on modeling of PV systems, optimizing the size, placement, operational cost and maximizing energy production of the PV panels. The inherent intermittency and uncertainty of power availability is one of the biggest challenges associated with PV integration. Studies are conducted to predict the generation patterns of PV modules. The solar power generated by the PV array is based on the intensity of the solar radiation which in turn depends on the extra terrestrial radiation. Probabilistic models are obtained in [36] for PV output prediction using the hourly extra-terrestrial radiation data and the hourly clearness index. Researchers have used Markov, statistical and fuzzy approaches for solar radiation modeling [37-39]. Reference [40] uses a back propagation artificial neural network (ANN) to predict the insolation level and then Genetic Algorithm (GA) is utilized to optimize the power generation of the PV system at a specified voltage level. In [41], ANN techniques are used for analyzing the effects of passing clouds on the PV output.

Studies [42-46] provide a good insight on modeling and sizing of PV panels for efficient operation. In [42], the authors propose a numerical method for the sizing of a PV system. Results indicated that under sizing provided an unreliable system whereas over-sizing caused unnecessary expenditure. The authors have also provided a cost analysis for the PV system setup and provided a time frame for break even analysis. A genetic algorithm based elitist strategy for optimal sizing of PV system is provided in [43].In [44], a combination of linear programming and dynamic programming is used for an optimal PV system size. In [45] a detailed PV model sizing technique is presented that takes into account parasitic inductances and capacitances. In [46] authors develop a mathematical model and a control scheme of three phase grid connected and optimally sized PV system and simulation in MATLAB/Simulink.

The energy production and economics aspects of PV systems are addressed in many research works. The design and installation of PV modules at residential and commercial sites on a New England pilot feeder is discussed in [47]. The PV production and the monthly variation in energy output are compared. [48] presents a probabilistic approach based on convolution technique for assessing the performance of 
utility interactive photovoltaic systems. The PV and load models are defined for hourly to yearly ranges and the time value of energy is assessed.

Another widely addressed topic is that of power converter configurations for PV systems. References [49-50] provide comprehensive surveys on different single-phase and three-phase converter circuits for PV applications. The new trends in power electronics for the integration of PV to the grid are presented. The various multilevel converter topologies using diode clamps, bidirectional switches, flying capacitors, three phase inverters and single phase $\mathrm{H}$ bridge inverters are compared and contrasted. The improvement in the total harmonic distortion and the reduction of current and voltage ripples are the most important research directions for future.

\subsection{Impacts of PV on Distribution systems}

The high penetration of PV into the system brings interesting implications for the utilities. Larger flows on a distribution system, (which is designed for one way power flow) may impact system regulation and protection. One of the major factors affecting voltage regulation is the reverse power flow. Since a significant amount of power is introduced at the lower end of the radial line, the line loading appears low to the voltage regulator which implies that the tap settings under the current scenario become irrelevant with the penetration of PV. The injection of power downstream from a fuse will not be detected because the traditional fuses are not designed with those capabilities. The larger the number of PV with inverters in the system, the greater the chances of islanding during which the PV continues to supply local loads after a utility fault. If the detection of islanding by the protection relays is not proper, then the inverters may remain on-line and pose serious threats to the equipment and to personnel. If the utility has sagging voltage levels due to the high demand conditions, inverters must be disconnected. Since the loads remain on-line, the utility may see an increase in demand, aggravating the chances of a blackout. Significant efforts are required in terms of studying these impacts for the successful integration of PV to the grid.

Many utilities have undertaken pilot studies to assess the impact of high penetration PV on their networks. [51] presents a study conducted by a utility in Spain about the potential problems arising from PV penetration. This study focused on the imbalance in loading and the voltage regulation on the feeders. In [52], the impact of solar PV systems on the Sacramento Municipal Utility District feeders is analyzed. Studies indicated that PV systems provided overall benefit to the consumers by reducing energy consumption. The voltage impacts were minimal at low penetration levels and were increased at peak PV penetration. The time difference between the maximum PV system output and the residential demand 
peak limits the local voltage regulation benefit on the secondary or primary distribution feeders in a residential area.

\subsubsection{Impacts on Power Flow and Voltage drop}

Solar photovoltaics have an impact on distribution feeder voltage and regulation. As the penetration level of solar PV increases, reverse power flow on the distribution feeder leads to voltage rise and hence violations of voltage boundaries defined by ANSI. In [53], case studies are conducted on a feeder to assess the performance of commonly used voltage regulation schemes under reverse power flow. The system performance with coordination of inverter and utility equipment is analyzed. [54-55] analyze the effect of PV system location on the voltage regulation of the feeder. The simulation results show that the power quality of the system can be improved by suitable location selection of the PV systems. [56-57] provide detailed studies of voltage variations and loss analysis on low and medium voltage feeders with PV interconnection.

Reference [58] is of particular interest as it provides a comprehensive report on the steady state and dynamic impacts of PV penetration on utility feeders. The steady state impacts included reverse power flow, voltage fluctuations, increased operations of LTC's, regulators and cap-banks as well as increase in losses. The dynamic effects included the effect of islanding, the sudden cloud cover and inverter operation on the feeder. A clear research direction and future need has been established by the authors which highlights the urgent need for systemic interconnection studies of PV with the utility.

\subsubsection{Impacts on reliability, security and the network}

Apart from power flow and voltage drop, the impacts of PV on the other system parameters have generated considerable interest. [59-60] provide a broad overview of the impacts of PV systems on the system voltage stability and frequency. The introduction of local PV to balance the loads negatively impacts the efficiency of short term load forecasting modules. The traditional peak load regulation concept is impacted by PV integration and must be changed. [61] analyses the impacts of the PV system on the distribution lines to see the effects of feeder loading. Results demonstrated that at low PV penetration levels the losses and the voltage profile of the line are improved whereas at higher PV penetration, the losses are substantially increased along long distribution line. In [62], analysis conducted on a feeder with high penetration PV showed that the network impacts of PV were dependent on the penetration level. Mitigation solutions such as battery energy storage system and STATCOM were provided to address the integration issues. 


\subsection{Price Based Unit Commitment}

In the deregulated power market, a particular type of unit commitment is used by the GENCO to optimize generation resources in order to maximize its profit, called the Price Based Unit Commitment (PBUC). In PBUC, satisfying load is no longer an obligation and the objective is of maximizing the profit from trading energy and Ancillary Services (AS) in the market. The distinct feature of PBUC is that the market price reflects on all market transactions indicating market price as the only signal that enforces a unit's ON/OFF status and generation dispatch. In day-ahead market GENCO runs PBUC based on forecasted energy and ancillary services price, and price uncertainty needs to be considered as it has direct impact on the expected profit. Several approaches have been used to solve this PBUC problem viz. Linear/Non-Linear/Dynamic Programming and other meta-heuristic techniques [63-64]. The PBUC problem has been approached using Lagrangian relaxation (LR) and Dynamic Programming in [65]. A tradeoff between LR and Mixed Integer Programming to solve the PBUC is presented in [66]. A hybrid technique involving LR and evolutionary programming has been used in [67]. [68-70] present intelligent techniques like multi-agent and particle swarm optimization for solving PBUC. In this paper the LR method with dynamic programming has been used to solve the PBUC problem.

These innovative bidding strategies need changes when Gencos have adopted distributed generation resources such as wind farms to their portfolio; to supplement coal/natural gas fired generation and meet green generation mandates thereby maximizing profits. Wind farms present an innovative and clean technology, but their output is intermittent. Wind farms are capital-intensive but have lower operating costs than fossil-fuel plants. Although wind power offers many possible benefits, it has many potential challenges to participate competitively in the current restructured electric industry [71-74]. These challenges can be broadly classified into four categories.

- Network: The network constraints include geographical locations of wind farms and the capacity of the line/cable infrastructure to extract power at medium and high voltages from remote wind farms.

- Availability: For a GENCO with a wind generation, wind power availability forecast is very essential as it has a direct impact on the system performance and stability. There are several techniques for predicting the quantity of intermittent wind power [75-76]. These techniques involve a combination of simulation, statistical and weather based methods. The impacts of wind power variability on system operating costs are not negligible [77].

- Operation: Large penetration of wind farms introduces significant operational difficulties depending on size and voltage. 
- Pricing: With the availability of wind, a suitable adjustment must be made in its pricing, and this is usually related to the uncertainty in wind power availability. Some approaches to calculate the market clearing price are presented in [78-80]. There are different approaches to handle the uncertainties in competitive electricity market: probabilistic, stochastic and fuzzy systems. Fuzzy sets have been successfully applied to power system operation and planning to simulate uncertainties [81-83]. 


\section{Chapter 3}

\section{Simulation Software}

This chapter briefly describes the various software simulation packages used in this thesis for modeling the utility distribution feeder and the photovoltaic system. The modeling of a distribution system requires specialized package that utilizes three phase unbalanced distribution power flow. OpenDSS was used for distribution power flow analysis. JAVA programming was used to convert the existing CYMDIST model to OpenDSS model. MATLAB/Simulink was used for the PV model. MATPOWER was used for the optimal power flow solution for the profit based bidding strategy of the Genco.

\subsection{OpenDSS}

The Open Distribution System Simulator (OpenDSS or simply DSS) is a comprehensive electrical system simulation tool for electric utility distribution systems. OpenDSS is an open source developed by the Electric Power Research Institute [84]. There are two available implementations of OpenDSS, a standalone executable platform and an in-process COM server DLL, which is designed to drive the OpenDSS from a variety of other platforms. The executable version consists of a basic User Interface on the DSS solution engine to help users develop scripts and view solutions. The basic user interface is a text scripting standalone user interface which is sufficient for most of the analysis. The COM interface can be used to design and execute custom solution modes and features of the simulator from any third party analysis programs like MATLAB, VBA, C\#, Python etc. OpenDSS can support all kinds of steady state analysis commonly performed for utility distribution systems. In addition, the most important advantage of OpenDSS is that it supports analysis with distributed generation integration and time series power flow. OpenDSS can be expanded by integrating user developed DLLs to the solution engine to meet future needs. Some of the important applications of OpenDSS are listed below.

- Distribution Planning and Analysis

- General Multi-phase AC Circuit Analysis

- Analysis of Distributed Generation Interconnections 
- Annual Load and Generation Simulations

- Wind Plant Simulations

- Analysis of Unusual Transformer Configurations

- Harmonics and Inter harmonics analysis

- Neutral-to-earth Voltage Simulations

- Development of IEEE Test feeder cases and DG models

- Loss evaluations with unbalanced loadings

- Transformer frequency response analysis

- Open conductor fault conditions with a variety of single phase and three phase banks

\subsubsection{OpenDSS Architecture}

The architecture of the OpenDSS engine is shown in Figure 3-1. The main simulation engine consists of a DSS executive that controls the distribution system simulation. The various distribution components are divided into five object classes, namely:

- $\quad$ Power Delivery elements

- $\quad$ Power Conversion elements

- Controls

- $\quad$ Meters

- General

Power delivery elements are multi-phased and transport energy from one point to another and the most common power delivery elements are lines and transformers. The conversion of electrical energy from one form to another is achieved by power conversion elements. These components can also store energy temporarily. Most of the power conversion elements have a single connection to the power system. The power conversion elements are modeled by defining their impedance or a complicated set of differential equations to model their current injections. The most common power conversion elements are loads and generators. 


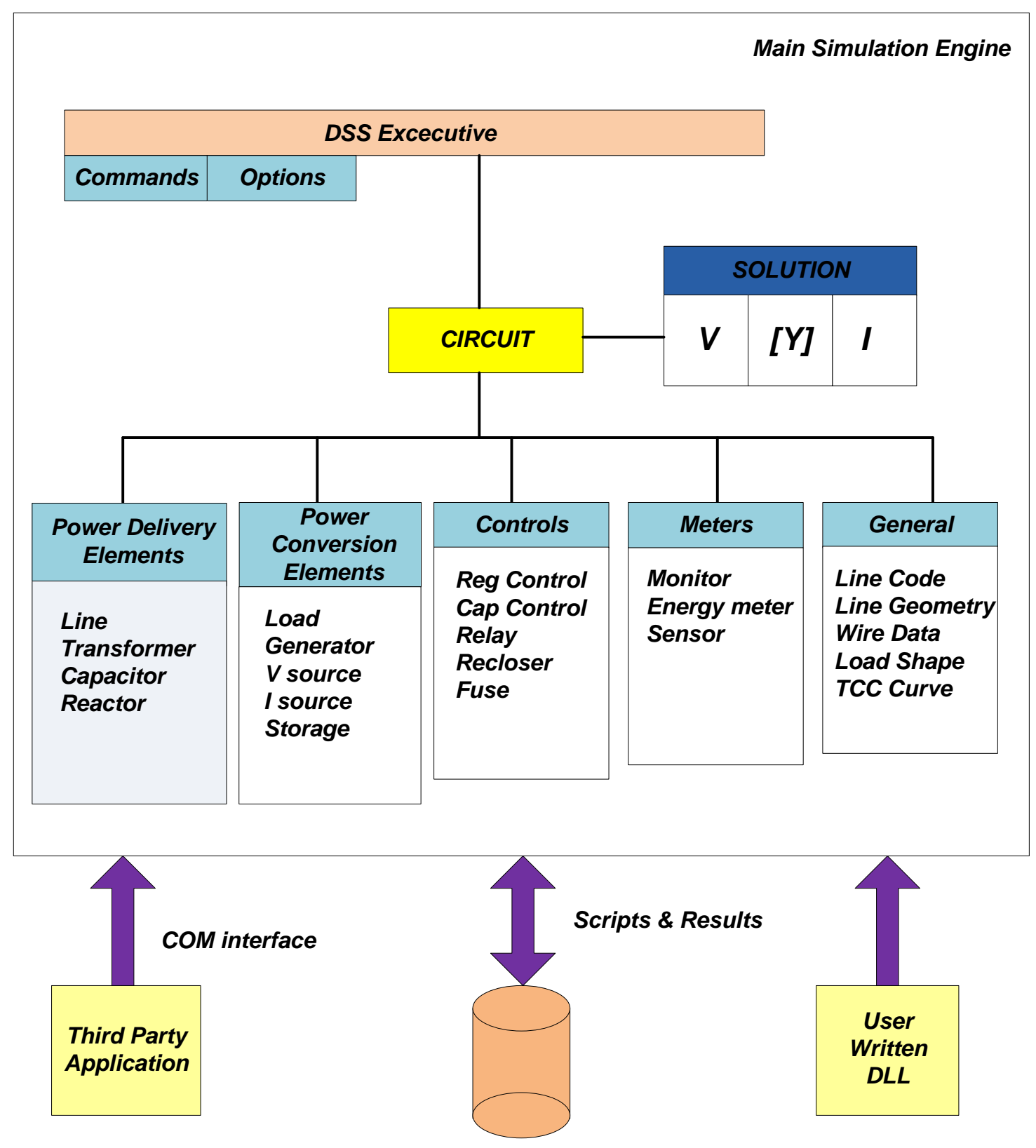

Figure 3-1 Open DSS Architecture

The Open DSS executive structure written in Delphi manages the creation and modification of the primitive $\mathrm{Z}$ and $\mathrm{Y}$ matrices for each element in the circuit and creation of the bus lists. The DSS executive also controls the collection of results through the meter elements and the execution of the control elements. The $\mathrm{Y}$ primitive matrices are fed to the sparse matrix solver, which constructs the system Y matrix. A no load power flow solution is used to arrive at an initial guess for the voltages. This is achieved by disconnecting the shunt elements and keeping the series power delivery elements. This is 
done to make all the phase angles and voltage magnitudes in a proper relationship. The iteration cycle is started by obtaining the current injections from all the power conversion elements and introducing them in the line vector. The sparse set of matrices is solved until the voltages converge to the specified tolerance. This simple iterative solution is found to converge well for most distribution systems that have an adequate capacity to serve the load. When performing daily or yearly simulations, the solution at the present time step is used as the starting point for the solution at the next time step. The solution typically converges in two iterations unless there is a large change in the load. After a converged solution, control iterations are performed if control actions are needed.

Although the power flow problem is the most common problem solved with the program, OpenDSS is best not characterized as a power flow program. It has its heritage from general purpose harmonic analysis tools and thereby gives it some unique and powerful capabilities. The program was originally designed to perform harmonics analysis aspects of distribution planning for distributed generation. OpenDSS is designed to perform a distribution power flow for small to medium sized feeders in which the bulk power system is the dominant source of energy. The circuit model can be multi-phased and/or a positive sequence model. The power flow can be executed in numerous solution modes such as the Single snapshot mode, Daily mode, Duty Cycle mode and Monte Carlo mode. The time duration can be any arbitrary time period and commonly, for planning purposes it can be 24-hour day, a month, or a year.

There are two basic power flow solution types provided by OpenDSS, namely the iterative and direct power flow solutions. In the iterative power flow mode, loads and distributed generators are treated as injection sources. For the iterative power flow mode, two power flow algorithms are currently employed namely, normal current injection mode and Newton current injection mode. The normal mode is usually faster, but the Newton mode is more robust for circuits that are difficult to solve. The normal mode is a simple fixed-point iterative method and works well for nearly all distribution systems. It is the preferred method for yearly simulations due to its speed. In the direct solution mode, the loads and generators are included as admittances in the system admittance matrix, which is then directly solved without iterating. Typically, power flow calculations use an iterative solution with non-linear load models, and fault studies use a direct solution with linear load models.

After the completion of the power flow, the losses, voltages, flows, and other information are available for the system, each component, and certain defined areas as shown in Figure 3-2. For each time instant the losses are reported as $\mathrm{kW}$ losses and energy meter models are used to integrate the power over a time interval. In this thesis OpenDSS is used to perform three phase unbalanced distribution power flow for the AEP feeder. 


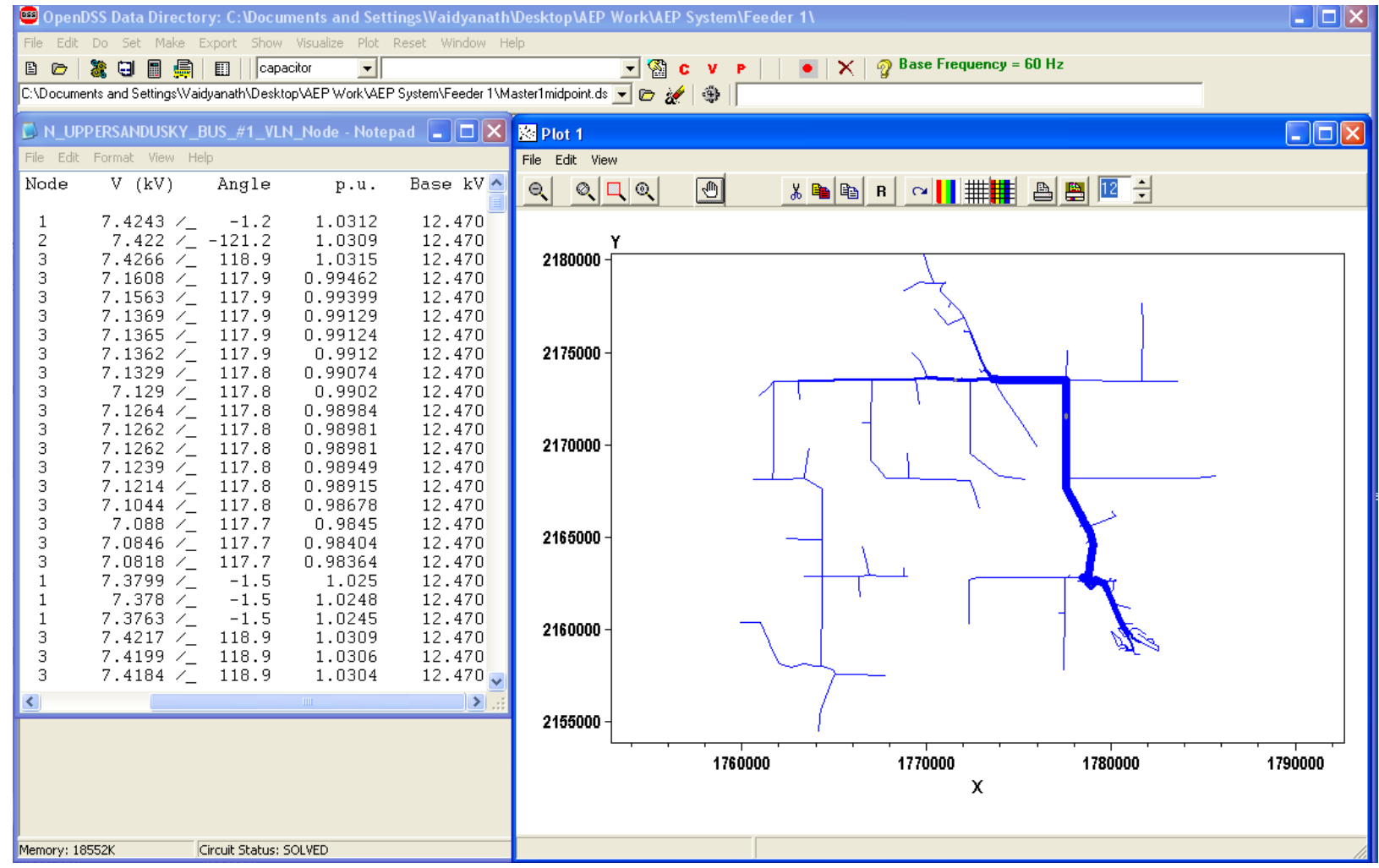

Figure 3-2 Open DSS Results Display

\subsection{CYMDIST}

CYMDIST is a commercial distribution system analysis tool developed by Cooper Power Systems Inc [85]. CYMDIST has the capability to perform analysis on balanced and unbalanced systems having three phase, two phase and single phase lines that are operated in radial, looped and meshed configurations. CYMDIST provides detailed graphical representation of the network, flexible user interface and extensive libraries as shown in Figure 3-3. 


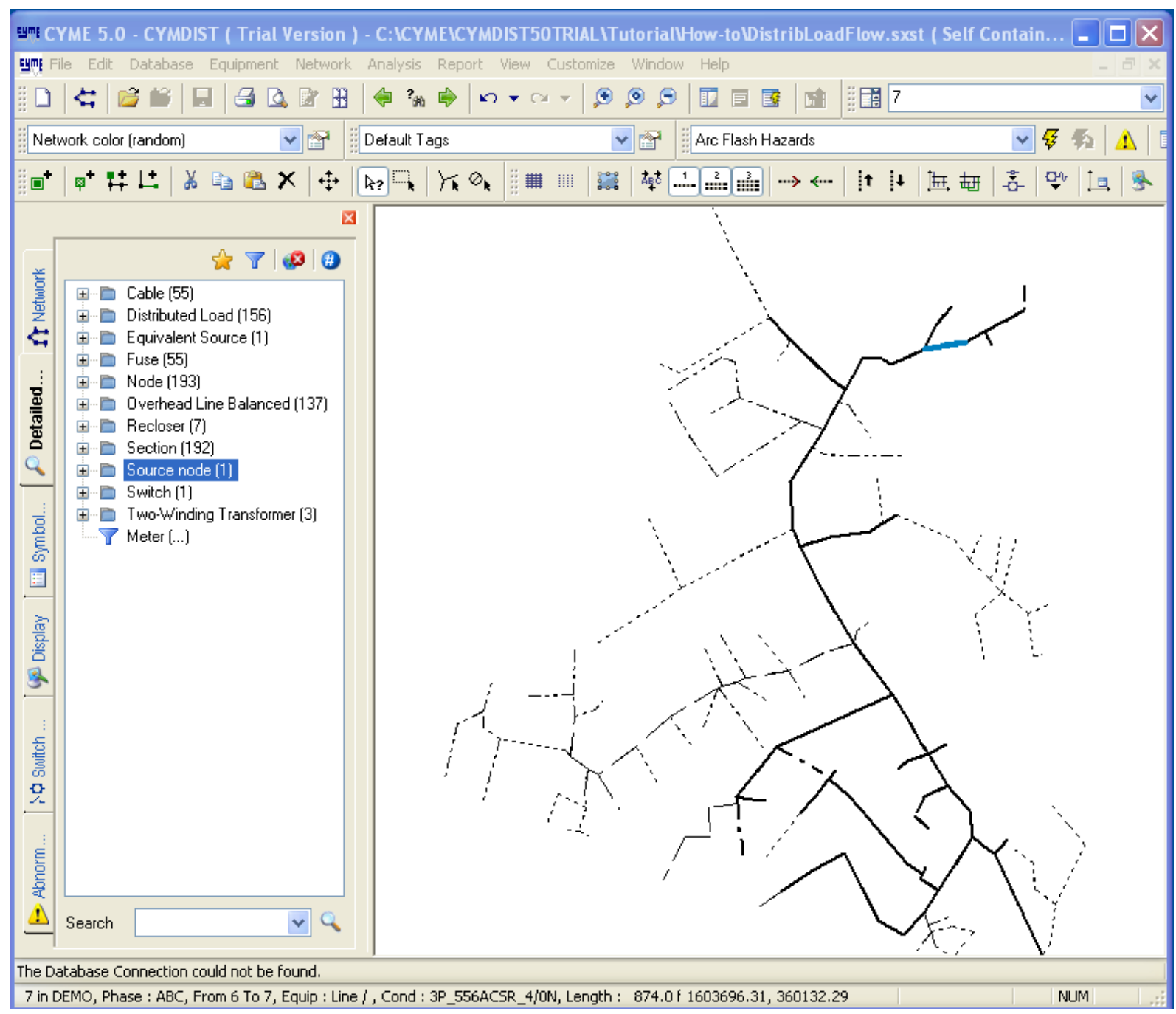

Figure 3-3 CYMDIST Network Display

CYMDIST provides a suite of applications composed of the network editor, analysis modules and user-customizable libraries to get the most detailed power flow solutions. CYMDIST is designed for planning studies and simulating the behavior of electrical distribution networks under different operating conditions and scenarios. It includes several built-in functions that are required for distribution network planning, operation and analysis. CYMDIST is also equipped with add-on modules to perform more in-depth analyses such as reliability analysis, contingency analysis, harmonic analysis and switching optimization. CYMDIST data resides in standard SQL tables and XML files can that be easily generated by third party applications. CYMDIST can be interfaced with other DMS, EMS and SCADA systems and a COM interface is available to support this. Apart from unbalanced power flow and voltage drop, some of the other functionalities of CYMDIST are 
comprehensive fault analysis, load balancing, load estimation and optimal capacitor placement, distributed generation modeling, service restoration, substation and sub-network modeling, secondary grid network analysis, arc flash hazard assessment and protective device coordination. CYMDIST includes a variety of report templates for all types of analyses and components as shown in Figure 34. In this thesis, the OpenDSS feeder model for AEP distribution feeder was developed based on the feeder model in CYMDIST.

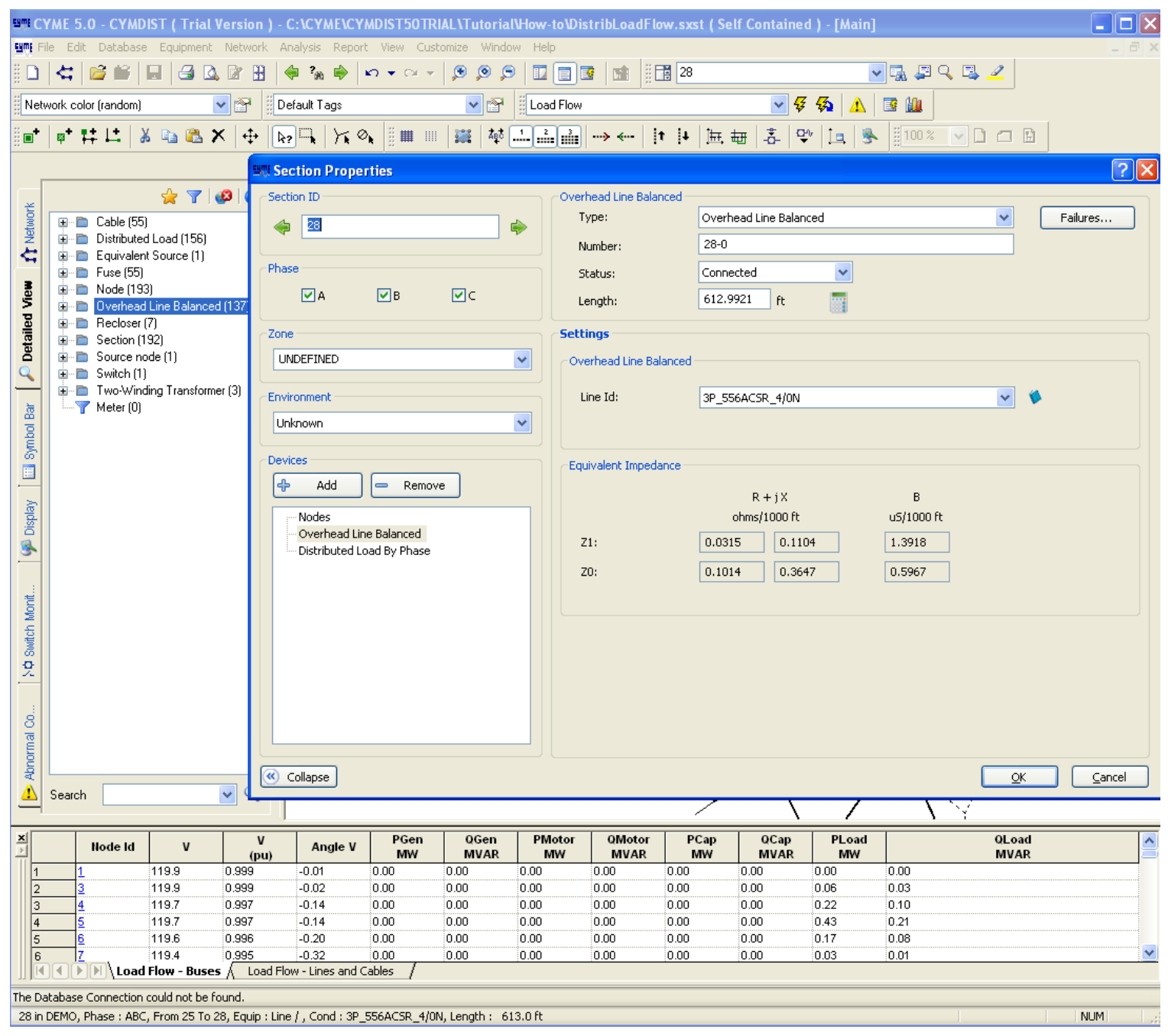

Figure 3-4 CYMDIST Report Display

\subsection{JAVA Programming}

In this thesis, JAVA is chosen as the programming language to convert the data from the CSV format of CYMDIST to the required format in OpenDSS. JAVA is a programming language and a computing platform developed by Sun Microsystems in 1995 [86]. Currently, it is the underlying 
platform that powers state of the art programs involving utilities, games and business applications. JAVA provides powerful text and file handling features which have been extensively used in this thesis work. The network information stored in MS Excel formats have been converted to the OpenDSS format by using the JAVA JExcel API [87]. The JAVA JXL API is a good way for Java programmers to access Microsoft Excel document formats. The JExcel API can read Excel data from a file stored on the local file system or from some input stream and create a Workbook format. The creation of the workbook format leads to the access of individual sheets and cells. The JExcel API provides extensive features to access and manipulate the data according to the user requirements.

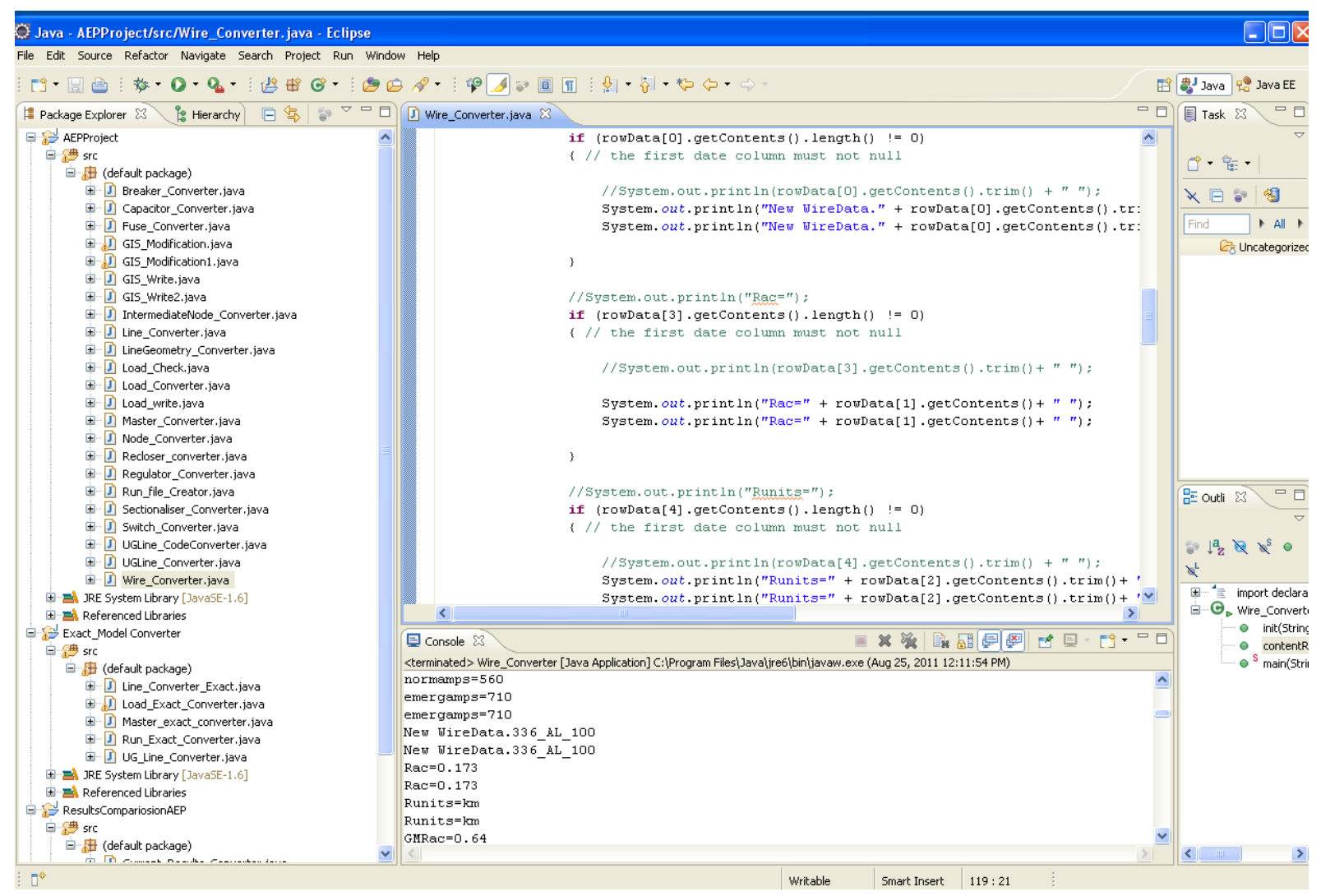

Figure 3-5 JAVA Eclipse IDE Environment.

Another feature of JAVA programming used in this work, is the Eclipse IDE for JAVA [88]. A snapshot of the IDE environment is shown in Figure 3-4. Eclipse is an open source developer community whose projects are focused on building an open deployment platform for JAVA. Eclipse provides a multi language software development environment comprising of a user interface called the IDE (Integrated development environment) and an extensible plug in system for users to develop 
their projects thereby reduces the programming burden for the users and provides error debugging. In its default form the Eclipse IDE is usually meant for JAVA developers to extend their abilities by installing plug-ins and development toolkits for tailored user requirements. In this thesis, Eclipse IDE is used for developing a JAVA project for the model conversion from CYMDIST to OpenDSS.

\subsection{MATLAB®}

MATLAB, Matrix Laboratory is a high level technical, scientific and numerical computing environment for development of algorithms, data analysis and numeric computation [89]. MATLAB provides toolboxes for solving specific engineering problems and has advanced 2-D and 3-D visualization features.

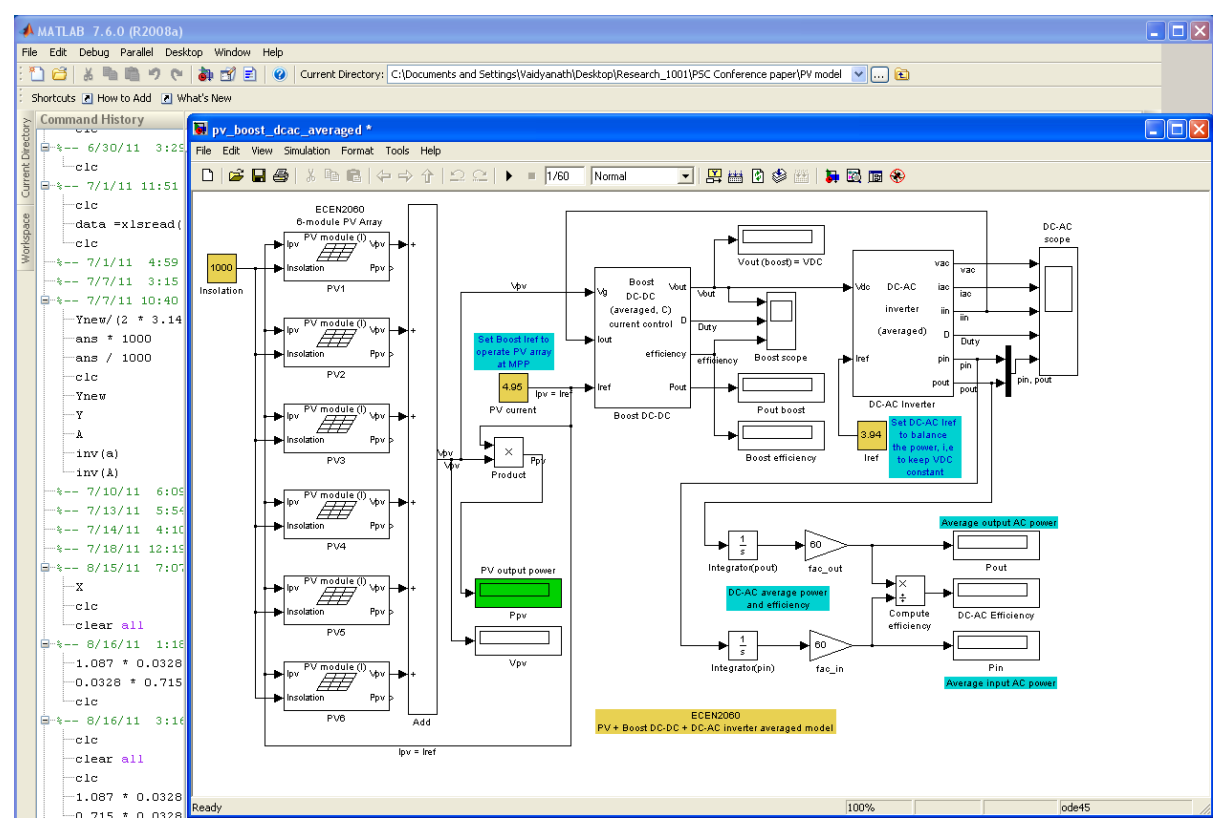

Figure 3-6 MATLAB Simulink model for PV system [90]

MATLAB is suited to engineering applications because its operations are based on matrices and vectors. The basic tasks like variable declaration, memory allocation and data type specification are faster than other computing languages. MATLAB also has object oriented programming concepts embedded into it and has a graphical user interface to create customized interfaces. Simulink, a commercial tool that is coupled with MATLAB is a tool for modeling, simulating and analyzing multi-domain dynamic systems [91]. It has a graphical block diagramming tool and a set of block libraries that contain most common engineering building blocks. Simulink is tightly integrated with the MATLAB environment and can be driven or scripted from MATLAB. Simulink is widely used in control theory and digital signal 
processing. In this thesis the PBUC optimization model was developed in MATLAB using Lagrangian relaxation and dynamic programming technique and the basic building block for the grid connected PV system model was built in MATLAB/Simulink.

\subsubsection{MATPOWER}

MATPOWER is a collection of MATLAB files for power system simulation and analysis applications. MATPOWER comprises of MATLAB m-files to solve load flow, optimal power flow and economic dispatch problems [92]. MATPOWER is a simulation package for the computational requirements of researchers and educators, developed by the Power Web project of Cornell University. MATPOWER is an open source tool and simulation cases can be developed in MATLAB environment. In this thesis, MATPOWER is used for optimal power flow solution of the power system, to determine the market clearing prices for the Genco with a distribution side windfarm. 


\section{Chapter 4}

\section{Modeling and Optimization}

In this chapter, comprehensive models of the utility feeder and its components are presented. A comparison of the models used in CYMDIST and OpenDSS for distribution system components are explained in detail. The photovoltaic system model is developed in MATLAB/Simulink for a distribution system to analyze the impacts on voltage profiles and losses. The optimization model of PBUC for a Genco with distribution side windfarm is formulated to assess the impacts of DG on power market operations.

\subsection{Introduction to OpenDSS Scripting}

The CYMDIST feeder model of AEP was available in two different formats, namely the XML format and CSV format. The XML format represented all the system data between a starting and ending tag. The CSV format provided system data in a text file using comma separated values. The network information and load information were provided in two separate files. The CYMDIST model contained network information providing the details regarding the location of the feeders, overhead and underground lines, cap banks, regulators, transformers and switches, and the load information providing the complete load details for all nodes of the feeder.

OpenDSS allows all functions to be carried out by a text based script. This makes OpenDSS easier for complicated circuits as DSS scripts can be configured to various data transfer formats. OpenDSS has specific command syntax for object definition and creation and parameters of each component can be provided in a random order. The OpenDSS compiler processes each parameter in a positional order and builds the component structure. Some commands require interpretation at more than one level. For example the NEW command is compiled by the main DSS Command interpreter and it passes the remainder of the string to the executive for adding new circuit elements. The DSS executive determines the type of element to add and confirms that it is a registered class. When the NEW command results in the instantiation of a DSS element, it is defaulted with reasonable default values. OpenDSS also supports a variety of delimiters, other special characters and array properties. The modeling of the various components in OpenDSS is described in the following sections. 


\subsection{Geographical Location of Feeders}

The geographical location of the feeders refers to the actual physical location of every element on the system and is represented by the $\mathrm{X}$ and $\mathrm{Y}$ co-ordinates. This is the first step in building of the utility feeder model in OpenDSS. The bus coordinates are specified in a file named Buscoords.dss'. In the utility data model, all the sections of the feeder are defined by the section ID. The section ID is the primary identifier for the location of all the components. From the section ID, the FROM" node and the ҒO" node can be deciphered. The conversion from the CYMDIST model to OpenDSS model for the bus-coordinates is shown in Figure 4-1.

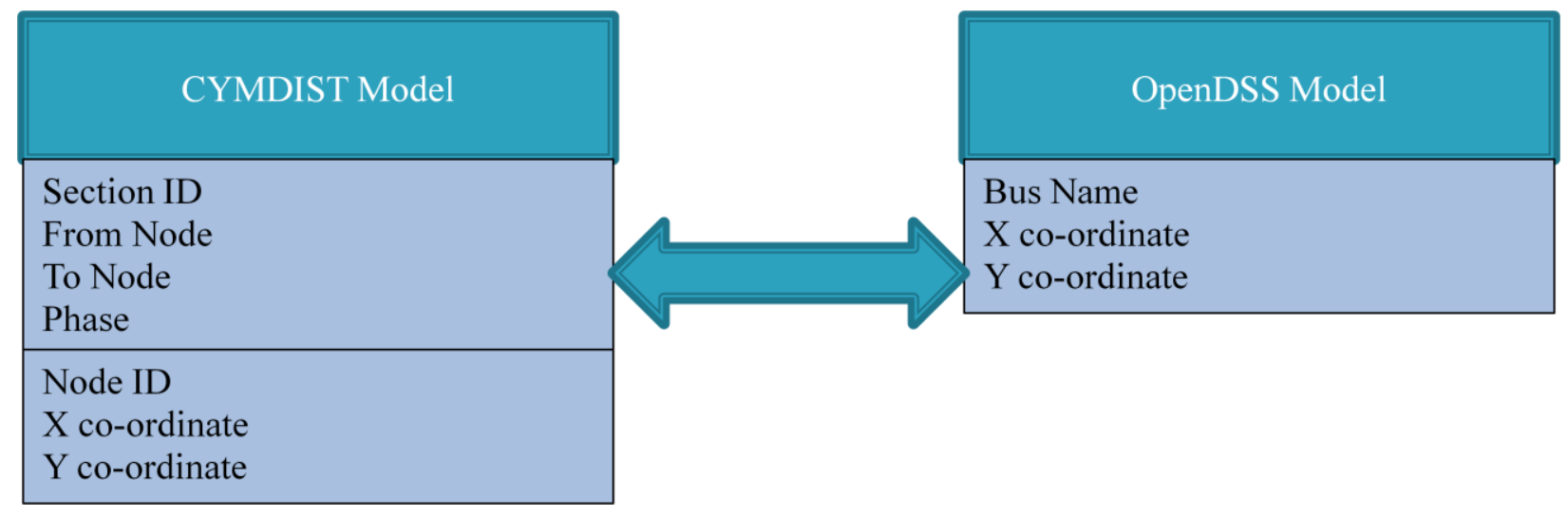

Figure 4-1 Comparison of the CYMDIST and OpenDSS models for Bus coordinates

The bus coordinates can be plotted as shown in Figure 4-2 for which OpenDSS provides a variety of plotting options. The plot may be multi colored with the thickness of the lines proportional to the quantity specified. 


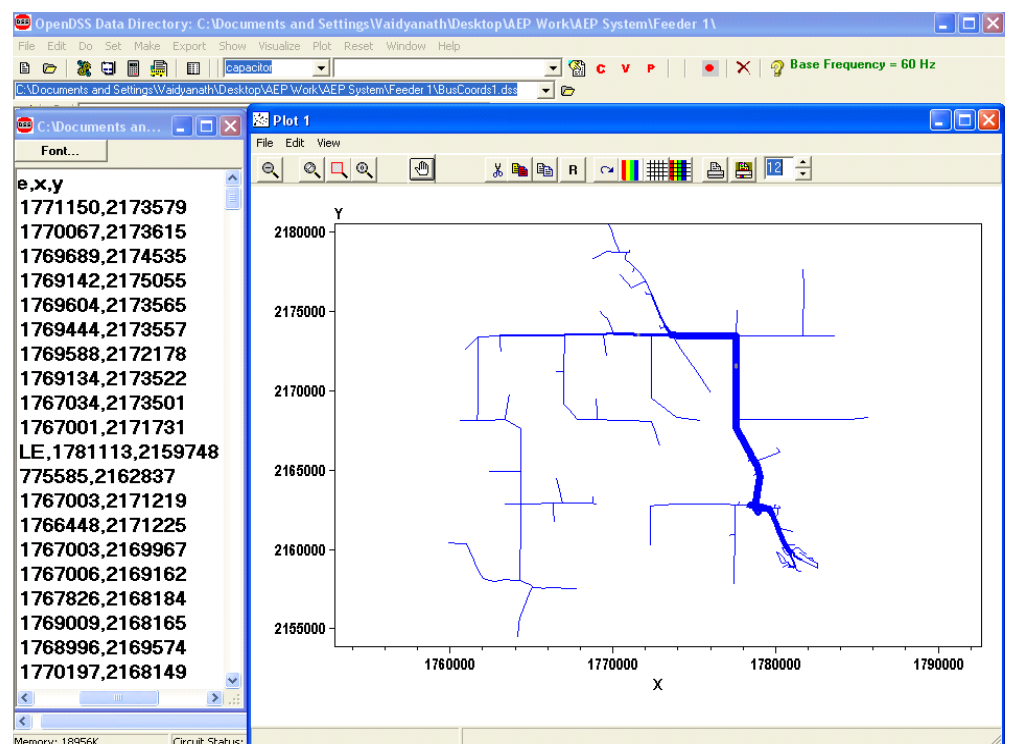

Figure 4-2 OpenDSS bus coordinates format and circuit plotting.

\subsection{Overhead and Underground Lines}

The overhead and underground line objects require detailed modeling in OpenDSS. The overhead line models have three components, namely the wire data, the line geometry and the actual line definition. Currently, OpenDSS does not have an inbuilt impedance calculation module for underground cables. Hence the impedance of the underground cables is specified in symmetrical components. The conversion from the CYMDIST model to OpenDSS model for the overhead and the underground lines is shown in Figures 4-3(a) and (b) respectively.

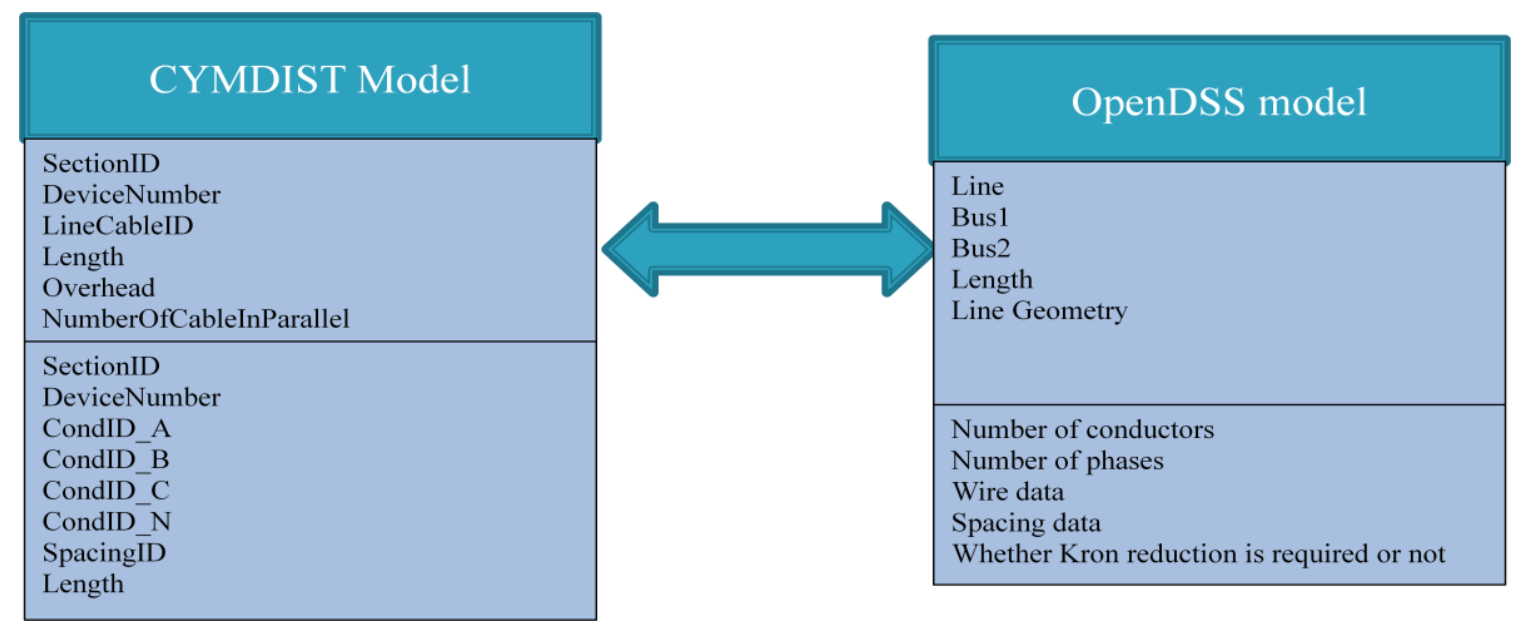

Figure 4-3(a) Comparison of the CYMDIST and OpenDSS models for overhead lines. 
CYMDIST Model

SectionID

Cable ID

Length

Positive Sequence Resistance

Zero Sequence Resistance

Positive Sequence Reactance

Zero Sequence Reactance

Positive Sequence Shunt Susceptance

Ampacity

\section{OpenDSS model}

UG Line

Bus1

Bus2

Length

Line Code

R0

R1

$\mathrm{XO}$

$\mathrm{X} 1$

C0

C1

Norm amps

Emergency amps

Base Frequency

Figure 4-3(b) Comparison of the CYMDIST and OpenDSS models for underground lines.

The equivalent line models require three files, namely the line definition, line geometry/ line code and the wire data. The format of line definition in OpenDSS is described as:

$\begin{aligned} & \text { New Line. <line name }>\text { bus } 1=<\text { bus1name }>.2 .4 \text { bus } 2=<\text { bus2name }>.2 .4 \text { length }=586.923 \text { units }=f t \\ & \text { geometry=geom4 (or linecode }=\text { code4) }\end{aligned}$

A unique device identifier is specified by the name". The connectivity model of the line, i.e., -FRM" and FO" nodes is specified by -buk" and bus2" respectively. The line is described as having the "B" phase and a neutral with the "B" phase represented by -.2 and the neutral represented by -.4. The length of the line and its corresponding units are specified. The details of the line geometry/ line code are presented in the subsequent files.

\subsubsection{Line Geometry}

Line Geometry is the class of objects used to specify the position of the conductors, the type of conductors and the Kron reduction settings. The different line geometry properties used in this thesis are listed in Table 4-1. 
Table 4-1 Line Geometry Properties

\begin{tabular}{|l|l|}
\hline \multicolumn{1}{|c|}{ Property } & \\
\hline Nconds & Number of conductors in the geometry \\
\hline Nphases & Number of phases. All other conductors are considered neutral and phased out \\
\hline Cond & This number identifies each conductor in the geometry \\
\hline Wire & Refers to the type of wire used and links to the Wire Data Class \\
\hline X,H & X coordinate and the height of the conductor in $\{$ mi $|\mathrm{kft}| \mathrm{km}|\mathrm{m}| \mathrm{Ft}|\mathrm{in}| \mathrm{cm}\}$ \\
\hline Reduce & Yes/No. Reduce out the neutrals using Kron Reduction formula. \\
\hline Normamps & Normal ampacity, amperes. \\
\hline Emergamps & Emergency ampacity, amperes \\
\hline
\end{tabular}

The format of line geometry in OpenDSS is described as:

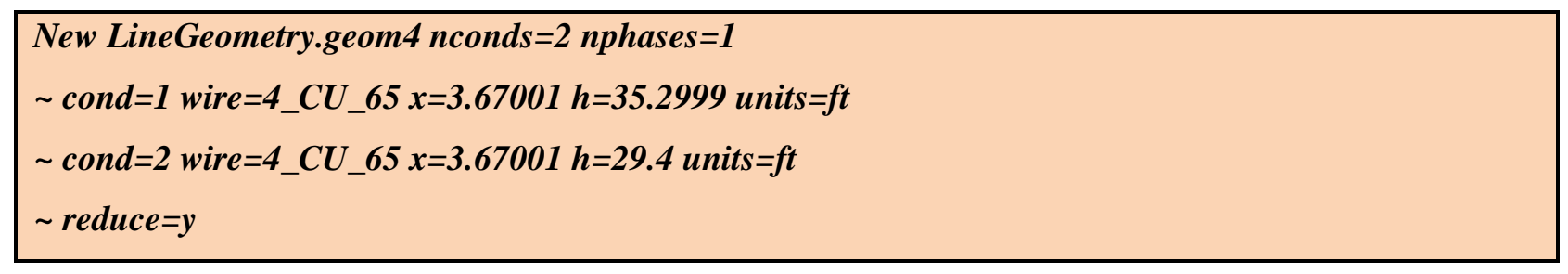

A unique overhead line geometry identifier is specified by - gom4". The - nonds" and —nphses represent the number of conductors and phases for this particular geometry. The individual conductors for this geometry are identified by $\multimap$ ond $=1$ " and - and $=2$ " respectively. The type of conductor used is described by - wi.", and its details are present in the wire data, as explained in Section 4.3.3. The " $\mathrm{x}$ " and "h" represent the $\mathrm{x}$-coordinate and y-coordinate positions of the conductor as measured from the ground level in units specified in the description. The Kron reduction technique is used to remove the elements corresponding to the neutral wire from the line impedance and susceptance matrices and can be enabled by setting the flag -aduce $=$ y".

\subsubsection{Line Code}

The Line code objects are general library objects that contain impedance characteristics for the lines and cables. Line code is used to specify the symmetrical impedance characteristics for underground cables. The impedance of a line is described by its series impedance and nodal capacitive admittance matrix. These matrices may be specified directly or they can be generated by specifying the symmetrical component data. OpenDSS can also perform a Kron reduction, reducing out the last conductor in the 
impedance matrices, which is assumed to be a neutral conductor. The different line code impedance properties used in this thesis are listed in Table 4-2.

Table 4-2 Line Code Properties

\begin{tabular}{|l|l|}
\hline \multicolumn{1}{|c|}{ Property } & \multicolumn{1}{c|}{ Description } \\
\hline Nphases & Number of phases. All other conductors are considered neutral and phased out \\
\hline R1 & Positive-sequence resistance in ohms per unit length. \\
\hline R0 & Zero-sequence resistance in ohms per unit length. \\
\hline X1 & Positive-sequence reactance in ohms per unit length. \\
\hline X0 & Zero-sequence reactance in ohms per unit length. \\
\hline C1 & Positive-sequence capacitance in nano-farads per unit length. \\
\hline C0 & Zero-sequence capacitance in nano-farads per unit length. \\
\hline Normamps & Normal ampacity, amperes. \\
\hline Emergamps & Emergency ampacity, amperes \\
\hline Base Freq & Base frequency at which the impedance values are specified. \\
\hline
\end{tabular}

The format of line code in OpenDSS is described as:

\begin{tabular}{|lllllr|}
\hline New Linecode.code4 & nphases $=3 \quad r 1=0.3489$ & $x 1=0.426198$ & $r 0=0.588811$ & $x 0=1.29612$ \\
c1=10.4308823411236 & $c 0=4.48501282215346$ & units $=k m$ & baseFreq=60 & normamps $=310$ \\
emergamps $=310$ & & & & \\
& & & & \\
\end{tabular}

A unique line code identifier is specified by —ode4". The —npmses" represents the number of phases for this line model. The line code contains the symmetrical component impedances. The positive sequence reactances and resistances are specified by — x'land -lr' in - bms per unit length" as defined by the units in the description. The $-Q$ " and +0 " properties represent the zero sequence reactances and resistances respectively in -ohis per unit length" as defined by the units in the description. The positive and zero sequence capacitances are specified by - $\mathbf{k}$ "and - 0 " " in — maofarads per unit length" as defined by the units in the description. The -bseFreq" property sets the base frequency for calculation of impedances. The normal and emergency current carrying capacity of the line is described by - namamps" and emergamps".

\subsubsection{Wire Data}

The wire data class defines the raw conductor data used to compute the impedances for line geometry calculation. The different parameters may be specified according to the units provided in the database and 
everything is converted to meters internally in OpenDSS. The different conductor properties used in this thesis are listed in Table 4-3.

Table 4-3 Conductor Properties

\begin{tabular}{|l|l|}
\hline \multicolumn{1}{|c|}{ Property } & \\
\hline Wire Name & Standard name of the wire \\
\hline Rac & Resistance at $60 \mathrm{~Hz}$ per unit length. \\
\hline Runits & Length units for resistance. Ohms per unit length per $\{\mathrm{mi}|\mathrm{kft}| \mathrm{km}|\mathrm{m}| \mathrm{Ft}|\mathrm{in}| \mathrm{cm}\}$ \\
\hline GMRac & Geometric mean radius of the conductor at $60 \mathrm{~Hz}$. \\
\hline GMR units & Units for the GMR $\{$ mi $|\mathrm{kft}| \mathrm{km}|\mathrm{m}| \mathrm{Ft}|\mathrm{in}| \mathrm{cm}\}$ \\
\hline Radius & Outside radius of the conductor \\
\hline Radunits & Units for the outside radius of the conductor $\{$ mi $|\mathrm{kft}| \mathrm{km}|\mathrm{m}| \mathrm{Ft}|\mathrm{in}| \mathrm{cm}\}$ \\
\hline Normamps & Normal ampacity, amperes. \\
\hline Emergamps & Emergency ampacity, amperes \\
\hline
\end{tabular}

The format of wire data in OpenDSS is described as:

New WireData.4_CU_65 Rac $=0.849 \quad$ Runits $=k m$ GMRac $=0.202$ GMRunits $=m$ Radius $=0.259$
Radunits $=$ cm normamps $=115$ emergamps $=180$

A unique wire data identifier is specified by -4_CU65". The physical properties of the conductor are described in this file. The "Rac" represents the resistance of the wire in - bms per unit length" and the units are specified by —Ruits". The geometric mean radius of the conductor and its units are specified by -GMRc" and -GMRnits" respectively. The radius of the wire and its units is specified by - Raids" and -Radurts" property. The normal and emergency current carrying capacity of the conductor is described by —nomamps" and emergamps". 


\subsection{Loads}

Load is a power conversion element that is the core of all power flow and voltage drop analysis. The load object is normally defined by its nominal $\mathrm{KW}$ and power factor. It may be modified by a number of multipliers like the yearly or the daily load shapes. The conversion from the CYMDIST model to OpenDSS model for the loads is shown in Figure 4-4.

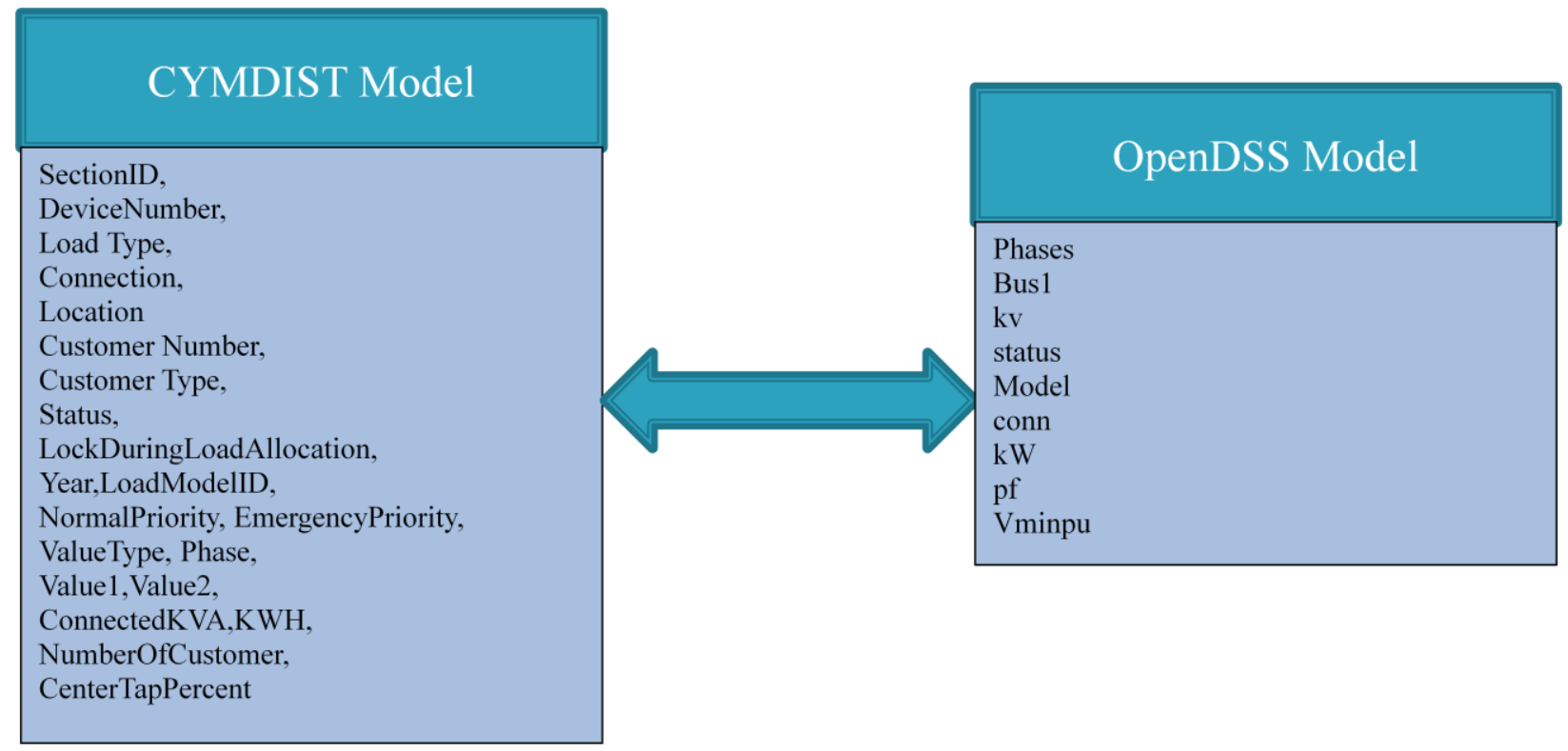

Figure 4-4 Comparison of the CYMDIST and OpenDSS models for Loads.

The default model for a load is a current injection source and hence the primitive impedance matrix contains only the impedance that exists from the neutral to ground for Wye connected loads. The loads are by default assumed balanced whereas unbalanced loads are represented by separate single phased loads. Loads can be of two types: Spot loads and Distributed loads. Spot loads are lumped at particular nodes whereas distributed loads are assumed to be uniformly distributed over a section. The modeling of distributed loads is described in detail in Section 4.4.1. The different properties of loads used in this thesis are listed in Table 4-4. 
Table 4-4 Load Properties

\begin{tabular}{|l|l|}
\hline \multicolumn{1}{|c|}{ Property } & \\
\hline Bus & Name of the bus to which the load is connected. \\
\hline Phases & Number of phases for the load. \\
\hline KV & Base voltage for the load in KV \\
\hline KW/KVAR & Nominal KW and KVAR for the load. \\
\hline Pf & Nominal power factor for the load. \\
\hline KVA & Definition of the base load in KVA. \\
\hline Model & $\begin{array}{l}\text { Defines how the load will vary with the voltage. In this thesis Model 1: Normal load } \\
\text { flow type load with constant P and Q }\end{array}$ \\
\hline Conn & Wye/Delta connection for the load \\
\hline Vminpu & $\begin{array}{l}\text { Minimum p.u. voltage for which the model is assumed to apply. Below this, it reverts } \\
\text { to constant impedance model. }\end{array}$ \\
\hline Vmaxpu & $\begin{array}{l}\text { Maximum p.u. voltage for which the model is assumed to apply. Above which it } \\
\text { reverts to constant impedance model. }\end{array}$ \\
\hline Xf KVA & $\begin{array}{l}\text { Rated KVA of the service transformer for allocating the loads based on connected } \\
\text { KVA at the bus. }\end{array}$ \\
\hline Allocation factor & Allocation factor for allocating the loads based on the connected Kva at the bus \\
\hline Class & Integer number that separates the load according to a particular class. \\
\hline Status & Fixed or Variable, to be modified by multipliers. \\
\hline NumCust & Number of customers to be served by this load. \\
\hline
\end{tabular}

\subsubsection{Distributed Load Model}

The majority of the loads specified by the AEP feeder model are distributed loads. The distributed loads are specified over a section of the feeder. The distributed loads can be mathematically considered as spot loads lumped at a particular spot in the section. According to the CYMDIST model, the load is lumped at $1 / 2$ of the distance from the starting point of the section. So the overhead/underground lines are split by creating a fictional node at $1 / 2$ of the distance and lumping the load at the midpoint as shown in Figure 4-5.

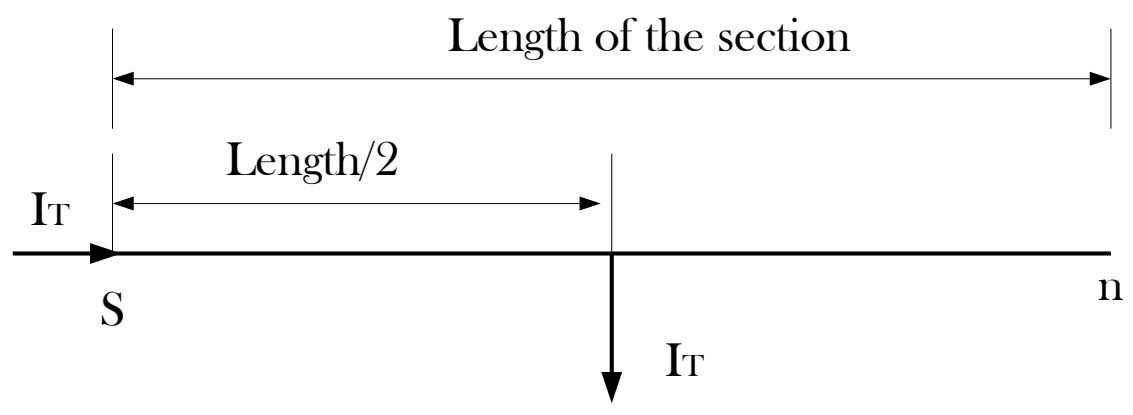

Figure 4-5 Distributed Load Model in OpenDSS. 
The format of equivalent distributed load model in OpenDSS is described as.

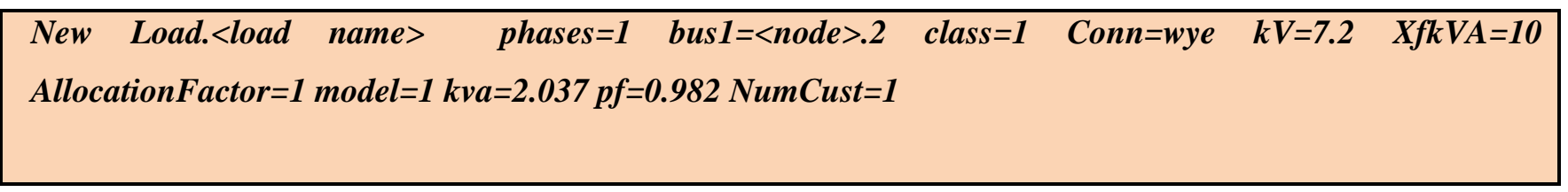

A unique load identifier is specified by -dad name". The load is described as connected to the - B phase of the node specified by — buk", with the "B" phase represented by ".2". The — pases" property describes the number of phases of the load. The type of connection and nominal KV, KVA and power factor are specified in the definition. XfKVA" and Allocation factor" are used for load allocation and represent the rated KVA of service transformer of the particular section and the load allocation factor based on the connected KVA respectively. The -elass" property represents an integer number to segregate the loads into residential, industrial, commercial and others. - Mddel=1" represents a PQ type load for power flow analysis. -NumCust" represents the number of customers for the load.

\subsection{Source}

The voltage source for the circuit is specified as a special power conversion element. It is considered special because, voltage sources are used to initialize the power flow solution and all other injection sources are set to zero. The conversion from the CYMDIST model to OpenDSS model for the voltage source is shown in Figure 4-6.

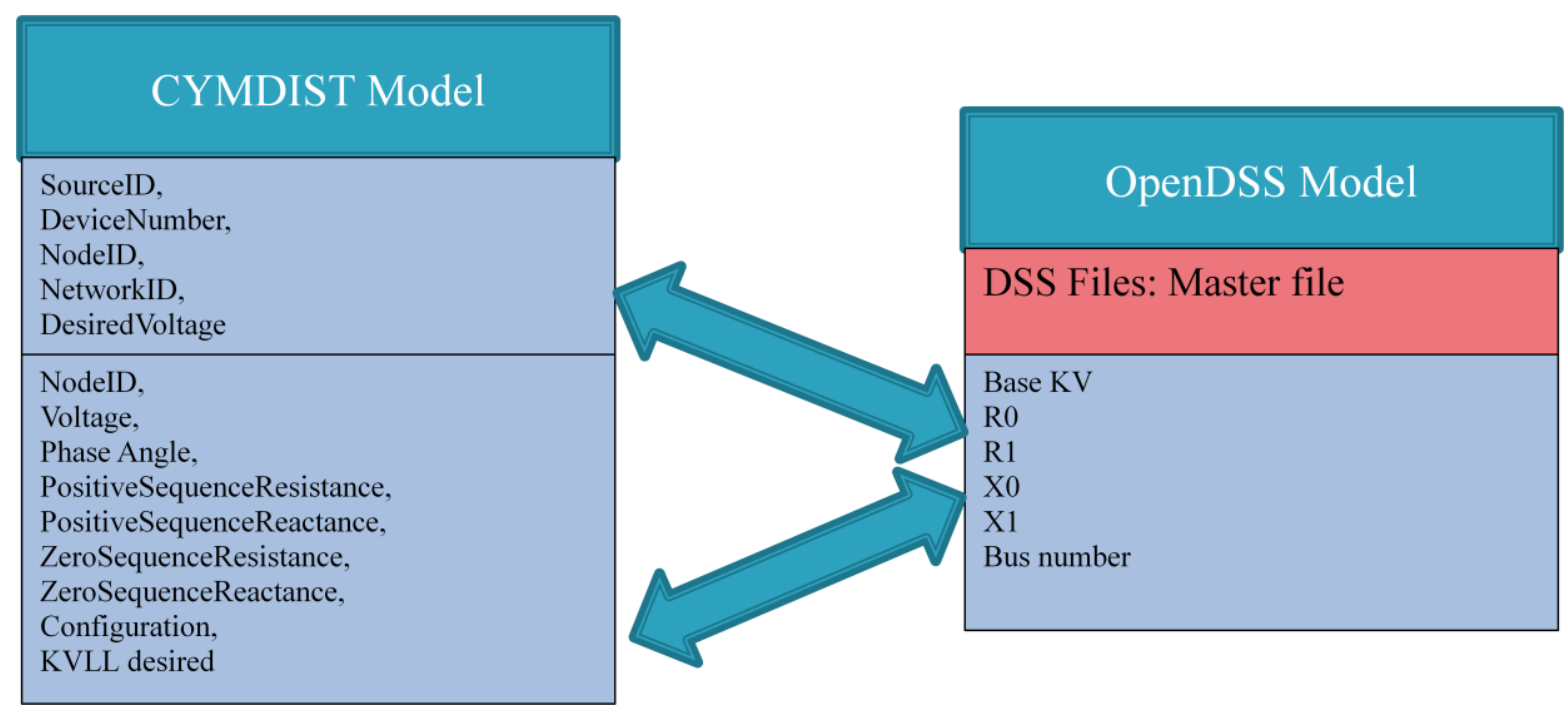

Figure 4-6 Comparison of the CYMDIST and OpenDSS models for Source.

The voltage source object is a two terminal, multi phase Thevenin equivalent, which represents a voltage source behind the impedance. The data for a voltage source is specified according to the common 
power system source definition standards: line-line voltage $(\mathrm{kV})$ and short-circuit MVA. The different properties for the voltage source used in this thesis are listed in Table 4-5.

Table 4-5 Voltage Source Properties

\begin{tabular}{|l|l|}
\hline \multicolumn{1}{|c|}{ Property } & \multicolumn{1}{c|}{ Description } \\
\hline Bus & The bus to which the source is connected. \\
\hline Base KV & Base/ Rated line-line KV \\
\hline p.u. & Actual per unit at which the source is operating \\
\hline Angle & Base angle, degrees of the first phase \\
\hline Frequency & Frequency of the source \\
\hline Phases & Number of phases of the source \\
\hline MVASC3/MVASC1 & 3 phase and 1 phase short circuit MVA \\
\hline R1 & Positive-sequence resistance of source in ohms. \\
\hline R0 & Zero-sequence resistance of source in ohms. \\
\hline X1 & Positive-sequence reactance of source in ohms. \\
\hline X0 & Zero-sequence reactance of source in ohms. \\
\hline
\end{tabular}

The format of voltage source in OpenDSS is described as:

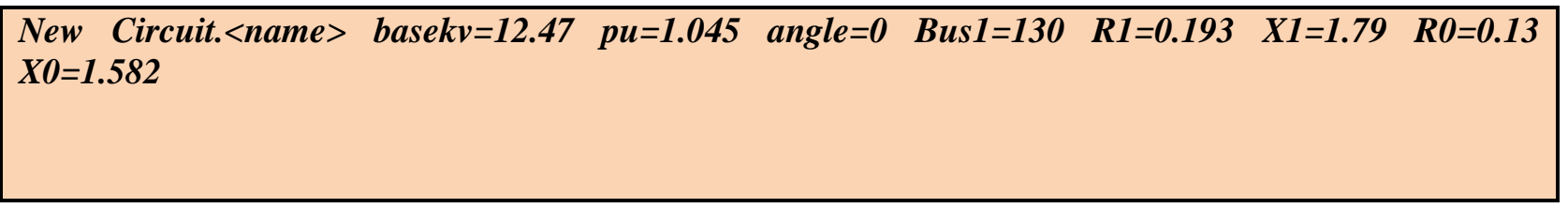

A unique circuit identifier is specified by -Bus". The definition of a voltage source is required when a new circuit model is created for analysis. The - brekv" represents the rated line to line voltage base for the circuit. The source operating voltage and angle are specified by the — pl." and the - agle" property. The positive sequence reactances and resistances of the source are specified by "x1"and $-\mathbf{r}$ " in ohms. The " $\mathrm{x} 0$ " and -0 " properties represent the zero sequence reactances and resistances of the source respectively in ohms.

\subsection{Regulator/Transformer}

The regulator is modeled as a transformer object in OpenDSS with a very low load loss and very low impedance and is implemented as a multi terminal power delivery element. The transformer object consists of two or more windings and the parameters of each winding are specified separately. The conversion from the CYMDIST model to OpenDSS model for the regulator is shown in Figure 4-6. 


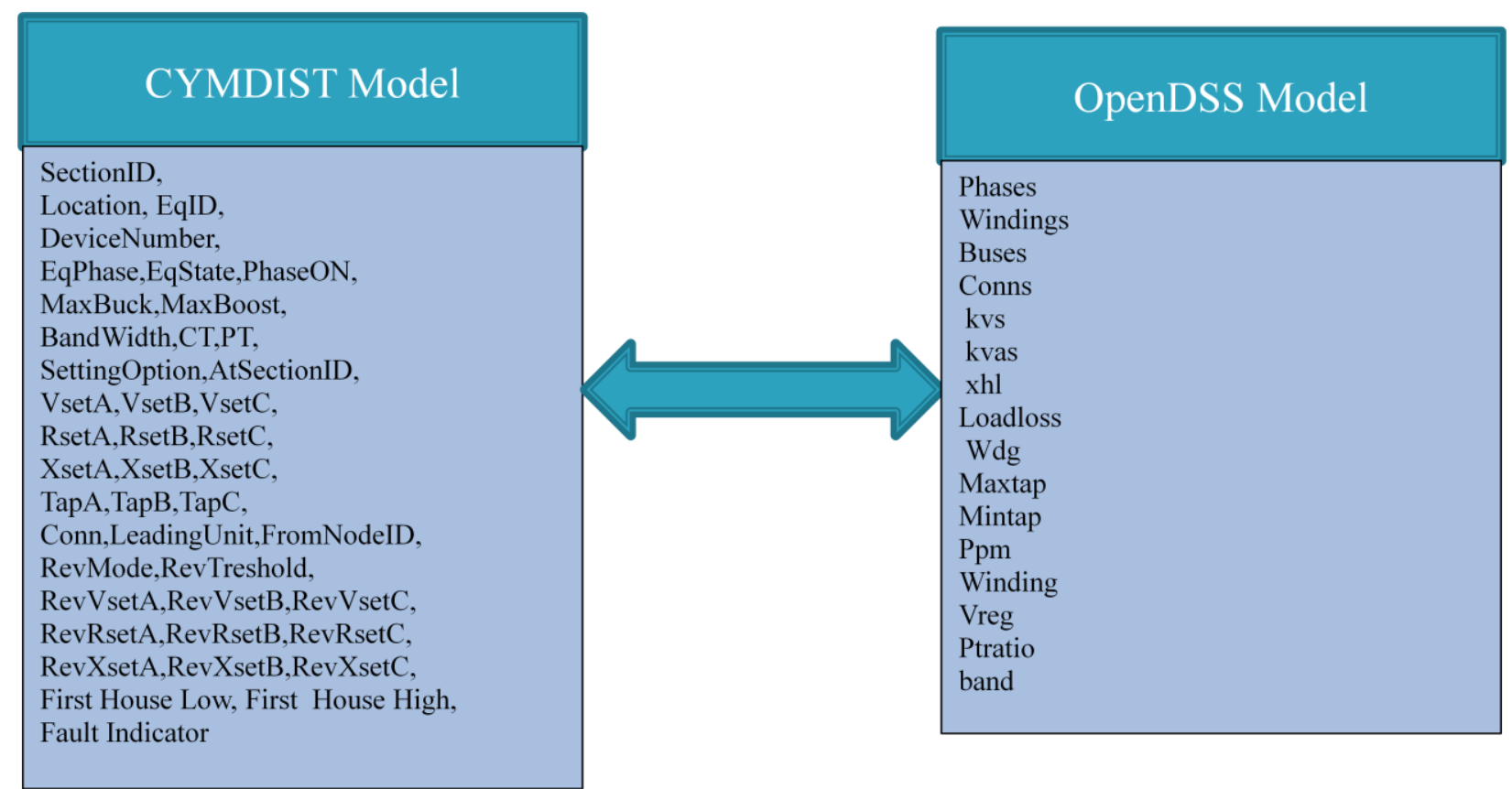

Figure 4-7 Comparison of the CYMDIST and OpenDSS models for Regulator.

The regulator is used to set the bus voltages within specified ANSI limits. Some commercial distribution analysis software, model regulator as a line drop compensator. The transformer object in OpenDSS achieves the same objective with small difference in the power flow and currents through the object. The different properties for the regulator used in this thesis are listed in Table 4-6.

Table 4-6 Regulator Properties

\begin{tabular}{|l|l|}
\hline Property & \multicolumn{1}{|c|}{ Description } \\
\hline Buses & Definition of bus to which the windings are connected \\
\hline Phases & Number of phases \\
\hline Windings & Number of windings \\
\hline Conns & Connections of this winding Wye/Delta \\
\hline kVA & Base KVA rating \\
\hline kV & Rated voltage of the winding \\
\hline Xhl & Percent reactance high-to-low winding \\
\hline Load loss & Percent load loss at rated load. \\
\hline Ppm & Parts per million for the anti floating reactance to be connected from each terminal to ground \\
\hline Vreg & Voltage regulator settings in volts for the winding to be controlled \\
\hline Ptratio & $\begin{array}{l}\text { Ratio of the Potential Transformer that converts the controlled winding voltage to the } \\
\text { regulator voltage. }\end{array}$ \\
\hline
\end{tabular}




\begin{tabular}{|l|l|}
\hline Ctprim & $\begin{array}{l}\text { Rating of the primary of the current transformer in Amperes, for converting the line amps to } \\
\text { control amps }\end{array}$ \\
\hline band & Bandwidth in volts for the controlled bus. \\
\hline
\end{tabular}

The format of the regulator bank model used in OpenDSS is described as:

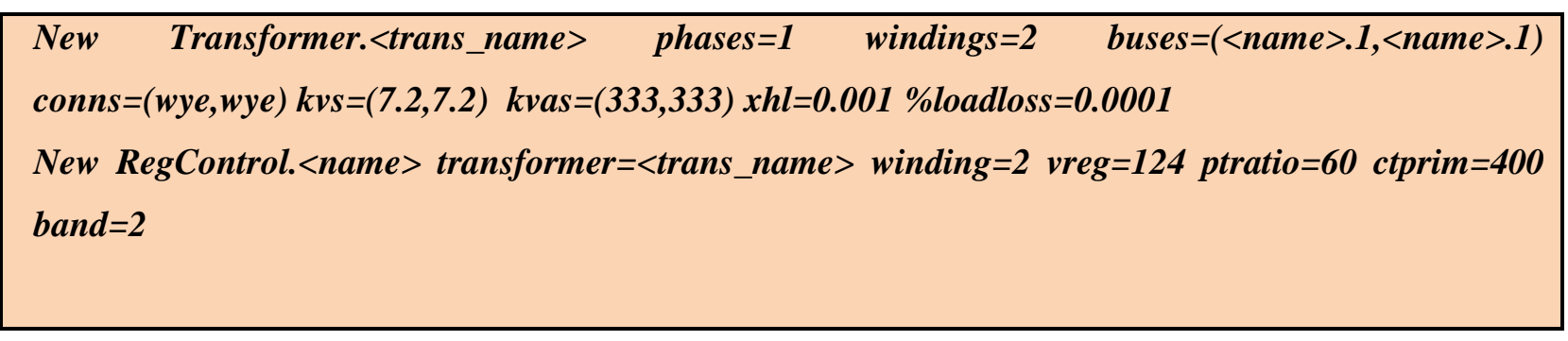

A unique transformer identifier is specified by rans_name”. A single phase transformer with two windings, their connection type and the buses to which it is connected is defined. The rated KV and KVA of the transformer are specified in the definition for the primary and secondary windings. The - xh' property defines the percentage reactance ratio of the primary to secondary windings and the — $\%$ adloss" property defines the load loss at the rated load for the transformer. The - RegControl" property defines the control settings of the regulator. The regulated voltage, bandwidth and the parameters of the CT and PT are defined.

\subsection{Capacitor Banks}

The capacitor model is basically implemented as a two terminal power delivery element. If the connection for the second bus is not specified, then the capacitor model is defaulted to a grounded wye shunt bank. The conversion from the CYMDIST model to OpenDSS model for the capacitor is shown in Figure 4-8.

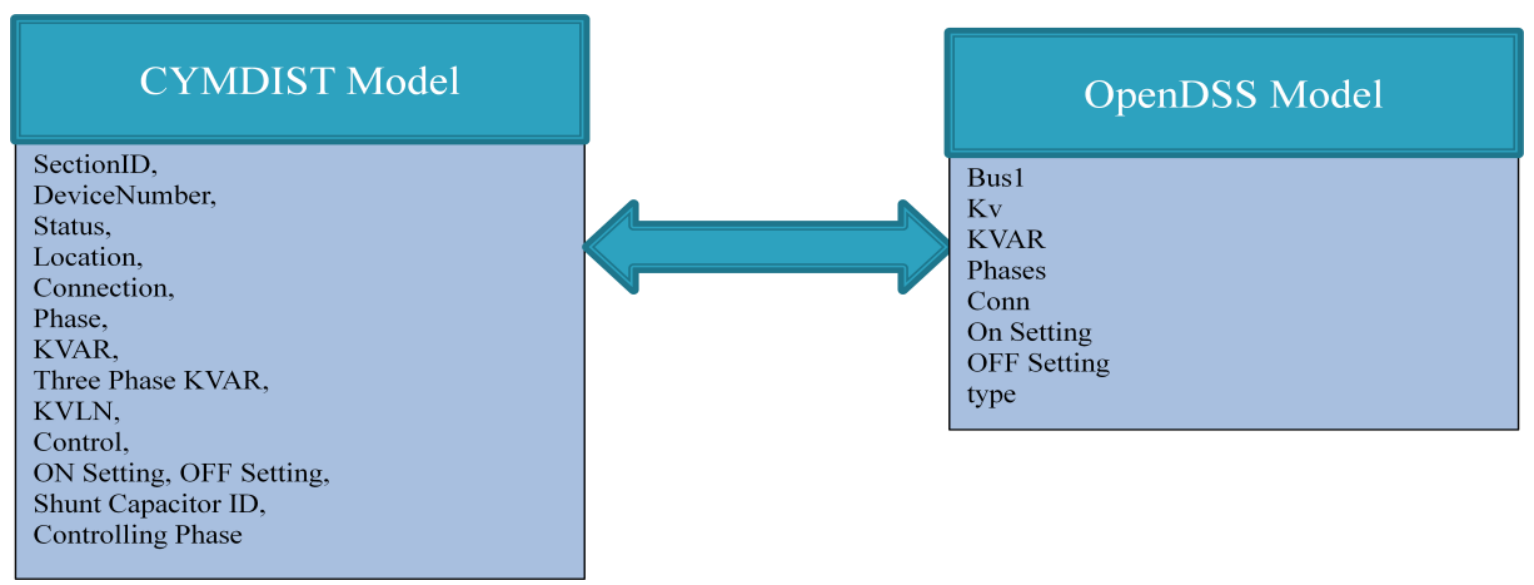

Figure 4-8 Comparison of the CYMDIST and OpenDSS models for Capacitor. 
The capacitor object can also be used as a series capacitor bank by specifying the second bus connection. By default, the capacitor bank is a multi step tuned filter bank. The Capcontrol property controls the capacitor bank by incrementing or decrementing the active step of the filter bank. The different properties for the capacitor used in this thesis are listed in Table 4-7.

Table 4-7 Capacitor Properties

\begin{tabular}{|l|l|}
\hline \multicolumn{1}{|c|}{ Property } & \\
\hline Bus & Bus to which the capacitor object is connected \\
\hline KVAR & Three phase/ Single phase KVAR rating of the Capacitor \\
\hline kV & Rated kV of the capacitor \\
\hline Phases & Number of phases \\
\hline Conn & Connection of the bank (Wye/Delta) \\
\hline Element & $\begin{array}{l}\text { The circuit element, typically line or a transformer, to which the capacitor control's PT and } \\
\text { CT are connected. }\end{array}$ \\
\hline Terminal & Number of the terminal of the circuit element to which the Capcontrol is connected \\
\hline Type & Control type settings for the capacitor. \{Current $\mid$ voltage $\mid$ kvar $\mid$ PF $\mid$ time $\}.$ \\
\hline ON setting & Value at which the Capcontrol aims to switch the capacitor ON \\
\hline $\begin{array}{l}\text { OFF } \\
\text { setting }\end{array}$ & Value at which the Capcontrol aims to switch the capacitor OFF \\
\hline Enabled & Yes/No Indicates whether the element is enabled \\
\hline CT phase & Number of the phase being monitored for current control \\
\hline PT phase & Number of the phase being monitored for voltage control \\
\hline
\end{tabular}

The format of the capacitor bank model in OpenDSS is described as:

New Capacitor. $\langle$ cap_name $>$ bus $1=\langle$ name $>$ kvar $=900 \mathrm{kv}=12.47$ phases $=3$ conn=wye

New CapControl. <control_name $\rangle$ Capacitor $=\langle$ cap_name $\rangle$ element=line. $\langle$ name $\rangle$ terminal $=2$

type $=$ current Onsetting $=43$ OFF setting $=33$ enabled $=y e s$ ctphase $=2$ ptphase $=2$

A unique capacitor identifier is specified by —ap_name". A three phase wye connected capacitor bank and its associated current control is defined. The location of the capacitor bank is specified by —bus" and the number of phases by the - phses" property. The KV and the KVAR settings of the capacitor bank are provided in the definition. The - Cap6ntrol" property defines the control settings of the capacitor. The $\rightarrow$ tpe=current" property defines a current controlled capacitor bank which can be turned ON and OFF when the current levels violate the -Osetting" and the -OFsetting" values. The -etphase" and -pthase" represent the individual phase to be controlled and in this case it is the $\mathrm{B}$ " phase as represented by " 2 ". 


\subsection{Switches, Breakers and Sectionalizer}

The switches, breakers and sectionalizers are elements used to control the connectivity of the circuit. In Open DSS, switches, breakers and sectionalizer elements are modeled as lines with a very low resistance and virtually zero reactance and capacitance. The operations of switch elements can be controlled manually in OpenDSS or by using the Swtcontrol property. The format of the switch, breaker and sectionaliser model in OpenDSS is described as:

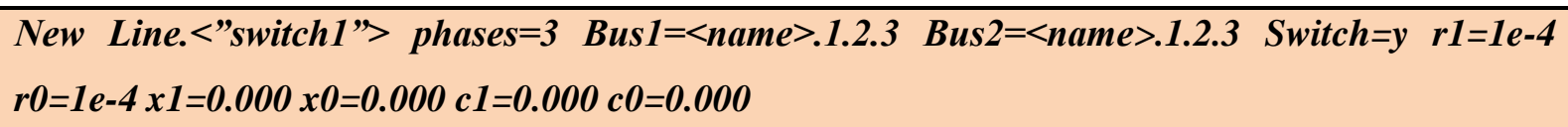

A unique switch identifier is specified by — witch1". The connectivity model of the switch, i.e., -FRM" and FO" nodes is specified by - buk" and - bs2" respectively. The switch is modeled as a line with a very low resistance and zero reactance as specified by the $-\mathbf{r}$ ", "r0", $-\mathrm{k}$ " and the $-\mathrm{x}$ 0properties. The switch can be enabled by setting the flag $\longrightarrow$ sitch=y".

\subsection{Fuses and Reclosers}

Fuses and reclosers are defined as control class elements in OpenDSS that control the current/voltage across an element and act on the monitored element according to the TCC curve specifications. A TCC curve object is defined as a curve consisting of an array of points and they are intended to model the timecurrent characteristics of overcurrent relays. The different properties for the fuses and reclosers used in this thesis are listed in Table 4-8.

Table 4-8 Fuse/Recloser Properties

\begin{tabular}{|l|l|}
\hline \multicolumn{1}{|c|}{ Property } & \multicolumn{1}{c|}{ Description } \\
\hline Monitored Obj & $\begin{array}{l}\text { The circuit element, typically a line, transformer, load, or generator, to which the Fuse } \\
\text { is connected }\end{array}$ \\
\hline $\begin{array}{l}\text { Monitored } \\
\text { Term }\end{array}$ & The terminal of the circuit element to which the Fuse is connected. \\
\hline Rated current & Multiplier or actual phase amps for the phase TCC curve. \\
\hline Switched Obj & Name of circuit element switch that the Recloser controls. \\
\hline Switched Term & $\begin{array}{l}\text { Number of the terminal of the controlled element in which the switch is controlled by } \\
\text { the Recloser. }\end{array}$ \\
\hline Num Fast & Number of Fast (fuse saving) operations. \\
\hline
\end{tabular}




\begin{tabular}{|l|l|}
\hline Shots & Total Number of fast and delayed shots to lockout. Default is 4. \\
\hline Phase trip & Multiplier or actual phase amps for the phase TCC curve. \\
\hline TDPhFast & Time dial for Phase Fast trip curve. \\
\hline TDPhDelayed & Time dial for Phase Delayed trip curve. \\
\hline $\begin{array}{l}\text { Reclose } \\
\text { intervals }\end{array}$ & Array of reclose intervals. \\
\hline
\end{tabular}

The format of the fuse model in OpenDSS is described as:

New Fuse. $<$ fuse name $>$ monitoredobj=.<name $>$ monitoredterm $=2$ ratedcurrent $=12$

A unique fuse identifier is specified by $\rightarrow$ fse name". The fuse typically monitors a line or a transformer which is specified by the - manitoredobj" property. The terminal at which the fuse is present is defined by the - mnitored term" property. The current carrying capacity of the fuse is defined in the ratedcurrent" property and if the current exceeds this value, the fuse object - lows out" to disconnect the downstream sections.

The format of the recloser model in OpenDSS is described as:

\begin{tabular}{|c|c|}
\hline $\begin{array}{l}\text { New Recloser. }<\text { recloser name }> \\
\text { switchedterm }=1 \quad \text { numfast }=1 \\
\text { recloseintervals }=(0.5,2,2)\end{array}$ & $\begin{array}{cccr}\text { monitoredobj }=<\text { name }> & \text { monitoredterm }=1 & \text { switchedobj }=<\text { name }> \\
\text { phasetrip }=200 \quad \text { tdphfast }=1 \quad \text { tdphdelayed }=1 & \text { shots }=4\end{array}$ \\
\hline
\end{tabular}

A unique recloser identifier is specified by recloser name". The recloser typically monitors a line or a transformer which is specified by the - manitoredobj" property. The terminal at which the recloser is present is defined by the - mnitored term" property. The effect of the recloser action can control an object other than the -mnitored object". The controlled object and the terminal at which the control action occurs are specified by the $\rightarrow$ witchedobj" and the $\rightarrow$ witchedterm" property. The definition of recloser action parameters includes the number of shots to lockout, actual phase currents according to the time current curve and the time dials for fast and delayed tripping.

\subsection{PV model in Simulink}

This section describes the PV model in MATLAB/Simulink used for analysis in this thesis. An individual PV module can be represented as a diode circuit shown in Figure 4-9, in conjunction with series and parallel resistances Rs and Rp respectively. PV cells have very distinctive $V$ - $I$ characteristics with respect to the change in the load impedance, solar irradiance and temperature. 


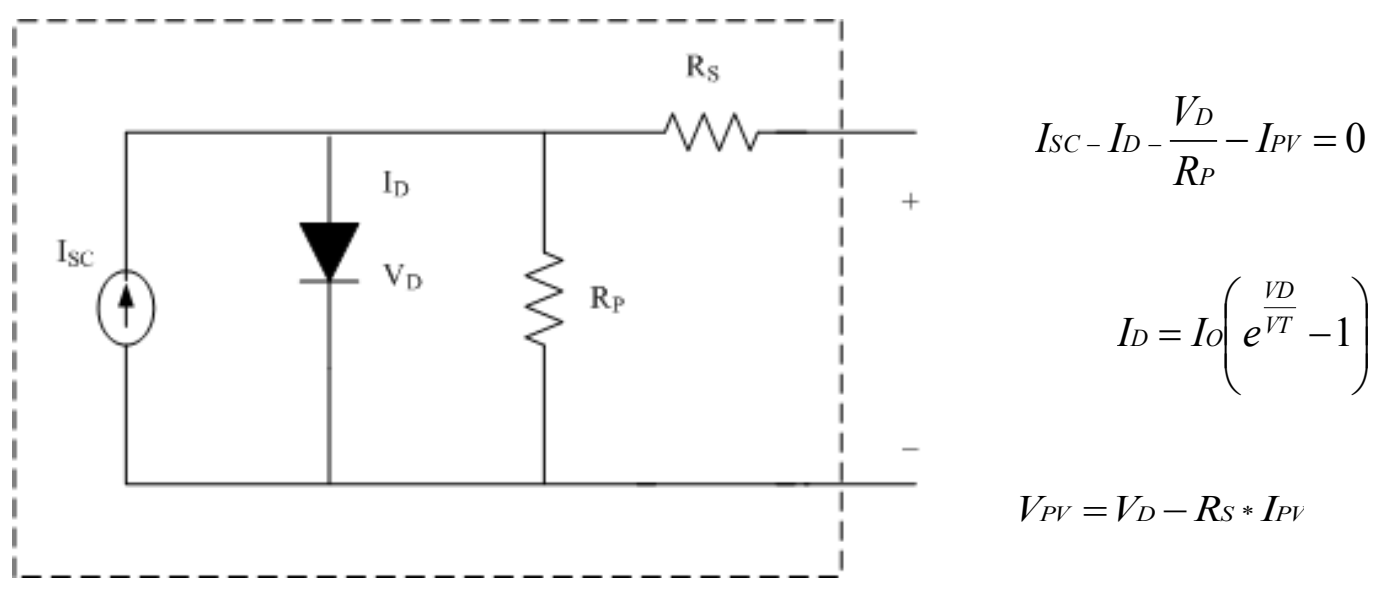

Figure 4-9 Individual PV Cell

Any increase in the solar insolation causes the short circuit current of the PV cell to increase, but it has very less impact on the open circuit voltage. However, an increase in the temperature leads to a small increase in the short circuit current and a small decrease in the open circuit voltage. Mathematically, the current and voltage characteristics for the PV module are expressed by equations (4.1), (4.2) and (4.3).

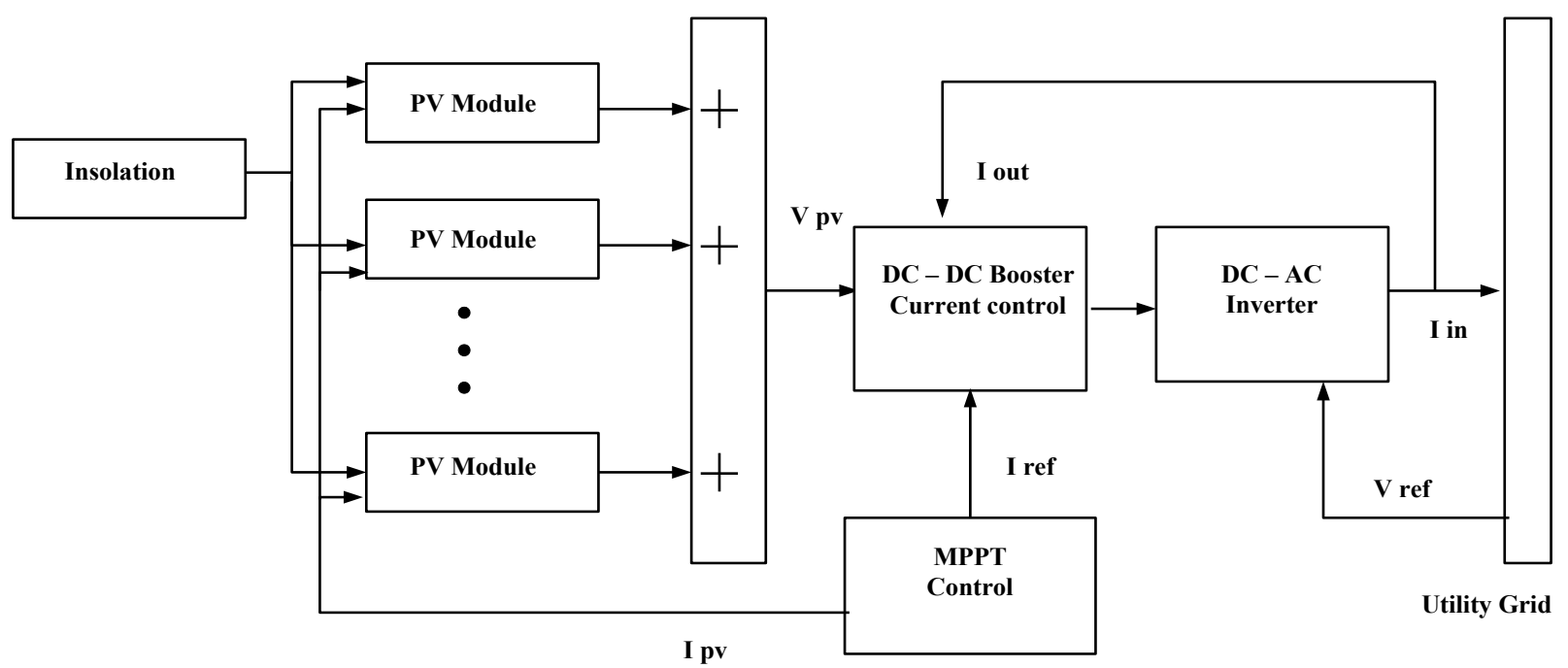

Figure 4-10 Grid Connected PV system.

A schematic of a grid connected PV system [92] is shown in the Figure 4-10. The PV system is a combination of many PV modules that are connected in series and parallel (by wiring them together) to obtain a suitable power point rating. $\mathrm{V}_{\mathrm{pv}}$ is the DC output voltage of the PV system which is then boosted and converted to $\mathrm{AC}$ voltage by the inverter. 


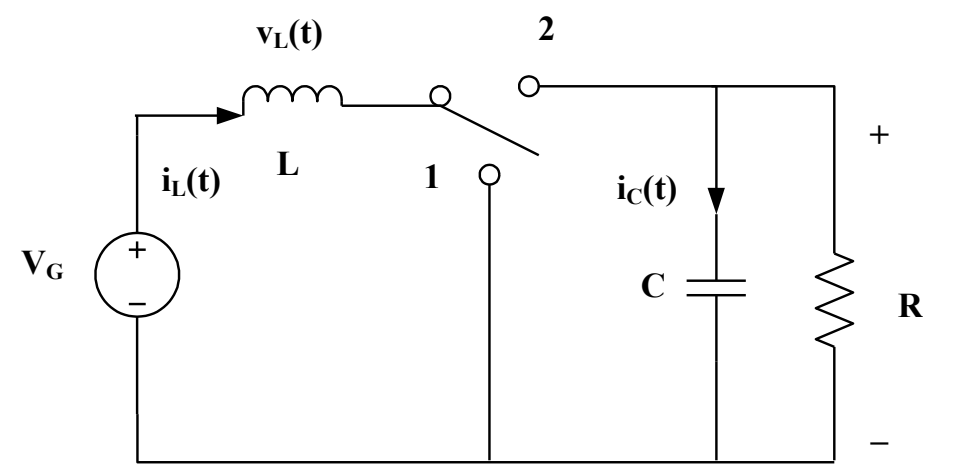

Figure 4-11 DC-DC Booster Model.

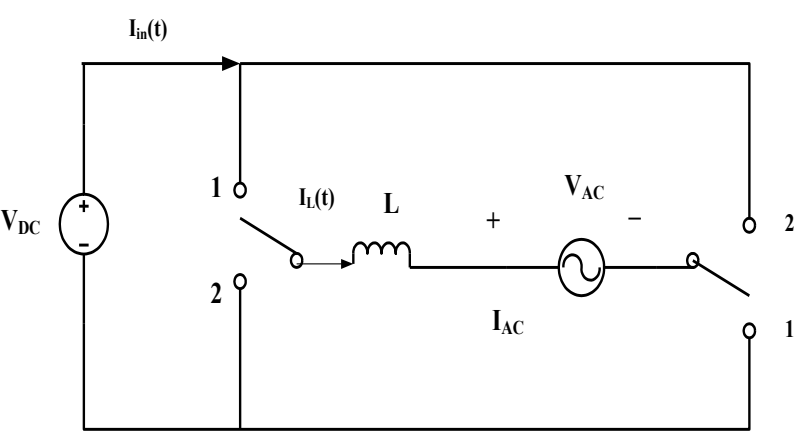

Figure 4-12 DC-AC Inverter Model.

To achieve the highest efficiency a Maximum Power Point Tracking (MPPT) system is used. This tracking system adjusts the inverter reference signal and thereby the DC voltage at the output of the solar array. Some of the MPPT techniques are the open circuit voltage method, the incremental conductance method, the ripple-based method and the Perturbation and Observation (P\&O) method. The method used in this thesis is based on (P\&O) algorithm, where at each cycle the voltage and current of the PV array are measured and the output power is compared with the value obtained in the previous cycle. The feedback from the MPPT controller adjusts the value of voltage to keep it constant. To boost the value of the voltage fed into the inverter, a DC - DC boost converter is used as shown in Figure 4-11. The voltage conversion ratio of the boost converter is given by equation (4.4), where $\mathrm{D}$ is the duty cycle. The boost converter in this paper is assumed to be lossless and hence it works as an ideal DC-DC transformer whose step ratio is electronically adjustable by changing the duty cycle.

$$
M(D)=\frac{V O U T}{V_{G}}=\frac{1}{1-D}
$$

The output of the inverter depends upon the solar irradiance and is affected by the temperature of the cells. The DC - AC inverter model is shown in Figure 4-12. The inverter operates as an AC currentcontrolled voltage source inverter which is synchronized with the phase of the line voltage automatically through a current-controlling reference signal that is synchronized with the line. This technique allows the inverter to control its power factor, real power, and reactive power. The inverter adjusts its reactive power to the line reactive power or reactive current demand signal. Real and reactive power accuracy is controlled to within $+/-2 \%$ of the rated demand. If the voltage or frequency (or both) of the line strays from its specified range, the inverter stops and disconnects itself from the line and the PV arrays. The 
typical inverter efficiency range is between $90-95 \%$ and depends on the power levels in the circuit. At lower power levels, the efficiency drops due to switching and other fixed losses while at higher power levels, conduction losses dominate.

Table 4-8 Typical Data Sheet Parameters

\begin{tabular}{|l|l|}
\hline Parameter & Value \\
\hline Short Circuit Current $\left(\mathbf{I}_{\mathbf{S C}}\right)$ & $5.45 \mathrm{~A}$ \\
\hline Open Circuit Voltage $\left(\mathbf{V}_{\mathbf{O C}}\right)$ & $22.2 \mathrm{~V}$ \\
\hline Rated Current at MPP $\left(\mathbf{I}_{\mathbf{R}}\right)$ & $4.95 \mathrm{~A}$ \\
\hline Rated Voltage at $\mathbf{M P P}\left(\mathbf{V}_{\mathbf{R}}\right)$ & $17.2 \mathrm{~V}$ \\
\hline Thermal Voltage $\left(\mathbf{V}_{\mathbf{T}}\right)$ & $0.026 \mathrm{~V}$ \\
\hline
\end{tabular}

The grid connected PV system model as described above was incorporated in MATLAB and the parameters used for simulation of PV system are as shown in Table 4-9. These parameters are measured for the solar panel at standard test conditions of $1 \mathrm{KW} / \mathrm{m}^{2}$ at $25^{\circ} \mathrm{C}$, and ASTM E-892 spectral irradiance standards. The V-I and P-V relations obtained from the PV model are highly nonlinear and depend on the solar irradiance incident on the PV array as shown in Figure 4-13(a) and Figure 4-13(b) respectively.
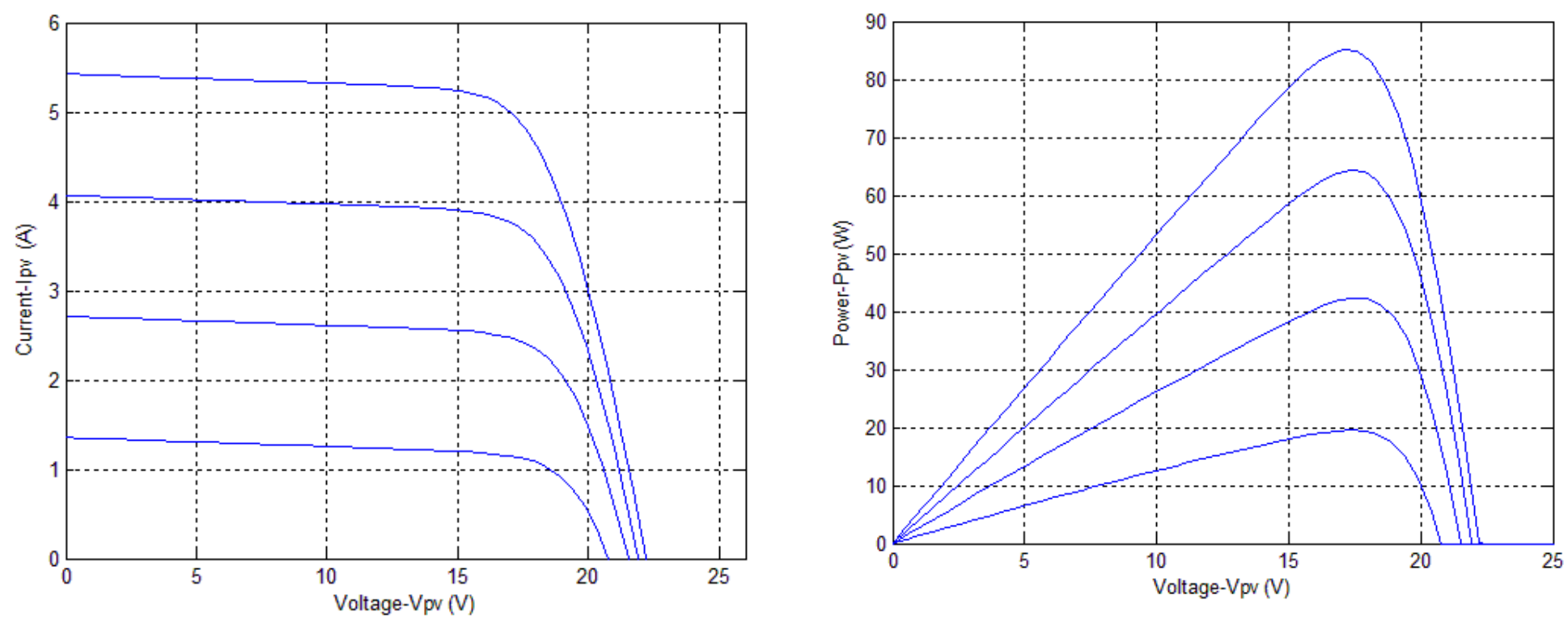

Figure 4-13(a) V-I characteristics for insolation ranging from $0.25 \mathrm{~W} / \mathrm{m}^{2}$ to $1 \mathrm{~W} / \mathrm{m}^{2}$; Figure 4-13(b) V-P characteristics for insolation ranging from $0.25 \mathrm{~W} / \mathrm{m}^{2}$ to $1 \mathrm{~W} / \mathrm{m}^{2}$ 
Utility scaled PV plants for example 1-10 MW are either directly connected to conventional feeders or o distribution substations via express feeders. These types of installations are three phase and typically require one or more interconnection transformers as shown in Figure 4-14. A MW-size PV-DG plant generally includes several power-electronic inverter modules connected in parallel—usually called power conversion systems

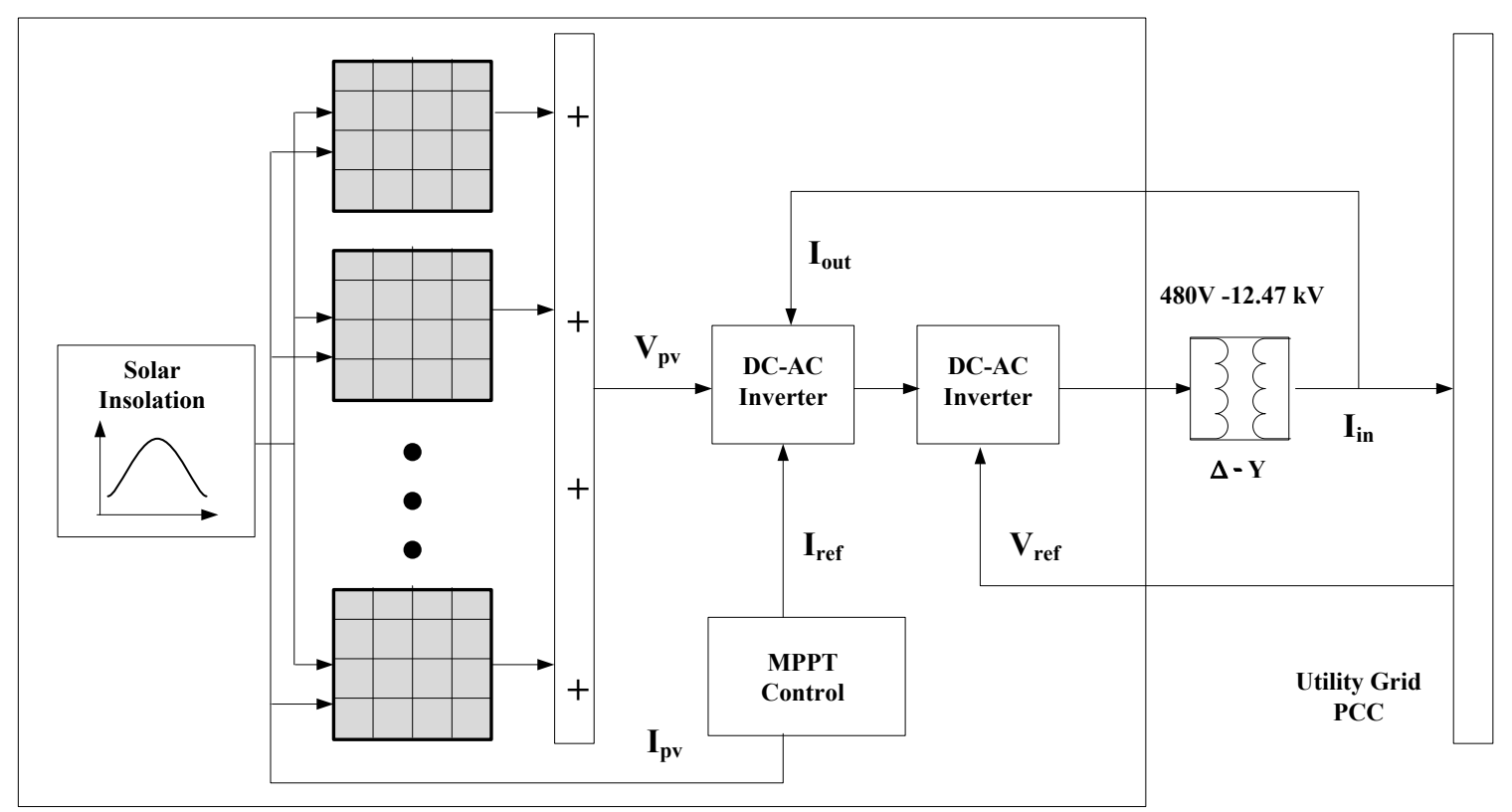

Figure 4-14 Utility scaled PV plant with interconnection transformer.

\subsection{Price Based Unit Commitment}

The Price Based Unit Commitment strategy for a Genco is a combinatorial optimization problem and the formulation is described in Section 4.11.1(b). The various constraints for the optimization problem and the dynamic programming to determine the hourly schedules are also discussed in subsequent sections.

\subsection{1 (a) Nomenclature}

$\mathrm{P}(j, i)-$ Generation of unit $j$ at time $i$

$\mathrm{R}(j, i)-$ Spinning reserve of unit $j$ at time $i$

$\mathrm{N}(j, i)$ - Non-spinning reserve of unit $j$ at time $i$ 
$\mathrm{RP}(j, i)-$ Energy price at the instant $i$

$\mathrm{RR}(j, i)-$ Spin price at the instant $i$

$\mathrm{RN}(j, i)-$ Non-Spin price at the instant $i$

$T^{o n}(j)-$ Minimum ON time of unit $j$

$T^{o f f}(j)-$ Minimum OFF time of unit $j$

$X^{o n}(j, i)$ - Time duration for which unit $j$ has been $\mathrm{ON}$ at time $i$

$X^{o f f}(j, i)$ - Time duration for which unit $j$ has been OFF at time $i$

UR (j) - Ramp up limit of unit $j$

DR $(j)$ - Ramp down limit of unit $j$

$\mathrm{L}(\mathrm{t}, \mathrm{ON})$ - Lagrangian function at time $i$ for ON status

$C L^{*}(\mathrm{t}, \mathrm{ON})-$ Optimal cumulative Lagrangian function at hour $i$ for the ON status.

$C L^{*}(\mathrm{t}, \mathrm{OFF})$ - Optimal cumulative Lagrangian function at hour $i$ for the OFF status.

$S U_{i, t}-$ Start up cost for unit $j$ at time $i$.

$S D_{i, t}-$ Shutdown cost for unit $j$ at time $i$.

\subsection{1(b) Problem Formulation}

The objective of PBUC is to maximize the profit (i.e. revenue minus cost) subject to all prevailing constraints [93]. For unit $j$ at time $i$, the objective function is given as

$$
\begin{gathered}
\sum_{i=1}^{n h r s}\left\{\left(-R P_{i}^{j} * P_{i}^{j}-R R_{i}^{j} * R_{i}^{j}-R N_{i}^{j} * N_{i}^{j}\right)+C\left(P_{i}^{j}+R_{i}^{j}+N_{i}^{j}\right)\right\} * I_{i}^{j}+\left\{\left(-R N_{i}^{j} * N_{i}^{j}\right)+C\left(N_{i}^{j}\right)\right\} *(1 \\
\left.-I_{i}^{j}\right)
\end{gathered}
$$


Equation (4.5) represents the objective function of PBUC in which $P(j, i)$ represents generation of unit $j$ at time $i, R(j, i)$ represents the spinning reserve of unit $j$ at time $i$ and $N(j, i)$ non-spinning reserve of unit $j$ at time $i$. The energy, spin and the non-spin prices at the instant $i$ are represented by $R P(j, i), R R(j, i)$ and $R N(j, i)$. The first part of the equation represents the profit when the unit is ON and the second part represents the profit when the unit is OFF. Here, profit represents revenue from the non-spinning reserve sales minus production costs and the cost of any energy purchases. Similarly, profit from bilateral contracts would also be included. The objective function for the total time period is

$$
\max F=\sum_{j} \sum_{i} F(j, i)
$$

The system constraints can be classified as $€ N "$ conditions and $€ F F "$ conditions and are represented by equations (4.7)-(4.10) and equations (4.11)-(4.13). The constraints represent the Genco's energy and reserve limits. The Genco may have minimum and maximum energy and reserve limits to participate in the market. Minimum and maximum generation, ramp rates, quick start and minimum ON-OFF time constraints are also represented

\section{Unit ON}

$$
\begin{aligned}
& N^{j}-\min \left(R_{\max }^{j}, P_{\max }^{j}-P^{j}-R^{j}\right) \leq 0 \\
& P_{\min }^{j} \leq P^{j} \leq P_{\max }^{j} \\
& R_{\min }^{j} \leq R^{j} \leq R_{\max }^{j} \\
& P_{\min }^{j} \leq P^{j}+R^{j}+N^{j} \leq P_{\max }^{j}
\end{aligned}
$$

\section{Unit OFF}

$$
\begin{aligned}
& P^{j}=0 \\
& R^{j}=0 \\
& N_{\text {min }}^{j} \leq N^{j} \leq N_{\text {max }}^{j}
\end{aligned}
$$


The minimum $€ \mathrm{~N}$ " time and $\Theta F F "$ time constraints are implemented in the dynamic programming routine and represented by equations (4.14)-(4.15) where $T^{o n}(j)$ represents the minimum ON time of unit $j$ and $T^{o f f}(j)$ represents the minimum OFF time of unit $j . X^{o n}(j, i)$ represents the time duration for which unit $j$ is ON at time $i$ and $X^{o f f}(j, i)$ represents the time duration for which unit $j$ is OFF at time $i$. The minimum $\mathrm{ON}-\mathrm{OFF}$ time constraints result in an expanded state transition diagram for the dynamic programming problem.

$\left[X^{o n}(j, i)-T^{o n}(j)\right] *\left[I_{i-1}^{j}-I_{i}^{j}\right] \geq 0$

$\left[X^{o f f}(j, i)-T^{o f f}(j, i)\right] *\left[I_{i-1}^{j}-I_{i}^{j}\right] \geq 0$

The ramp up and ramp down constraints of the system can be represented by equations (4.16) and (4.17) respectively where UR $(j)$ represents the ramp up limit of unit $j$ and DR $(j)$ represents the ramp down limit of unit $j$.

$P_{i}^{j}-P_{i-1}^{j} \leq U R(j)$

$P_{i-1}^{j}-P_{i}^{j} \leq D R(j)$

\subsubsection{Dynamic Programming}

In dynamic programming each unit of the Genco is considered individually for the UC problem, thereby increasing the dimension of the problem. Figure 4-15 shows the stages of the dynamic programming where the nodes represent states and the line joining the nodes represents time periods. 


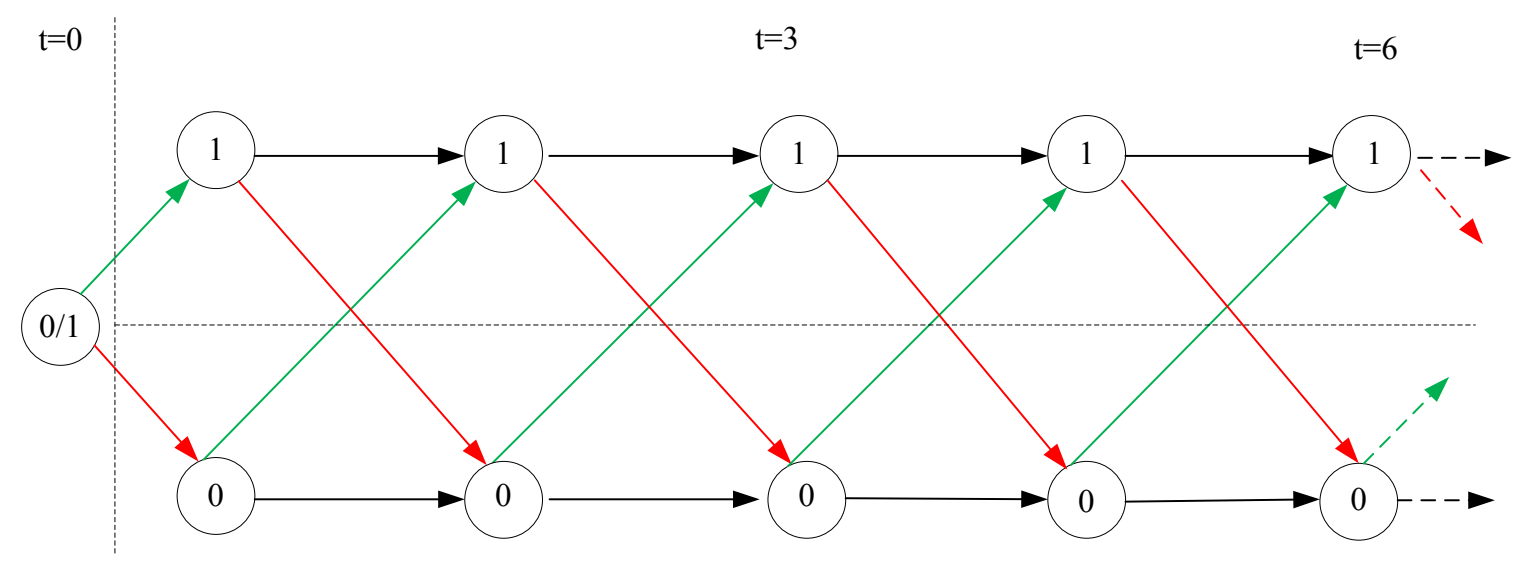

Fig.4-15 Stages in Dynamic Programming

Dynamic Programming consists of two stages namely forward search and backward search. The forward stage is used to find the optimal cumulative Lagrangian value at every hour for each state while the backward search is used to find out the optimal commitment trajectory. Mathematically the dynamic programming problem can be formulated by equations (4-18) and (4-19) where L (t, ON) represents the Lagrangian function at time $i$ for $\mathrm{ON}$ status, $C L^{*}(\mathrm{t}, \mathrm{ON})$ represents the optimal cumulative Lagrangian function at hour $i$ for the $\mathrm{ON}$ status and $C L^{*}(\mathrm{t}, \mathrm{OFF})$ represents the optimal cumulative Lagrangian function at hour $i$ for the OFF status. The startup cost for unit $j$ at time $i$ and shutdown cost for unit $j$ at time $i$ can be represented by $S U_{i, t}$ and $S D_{i, t}$.

$C L^{*}(t, O N)=\min \left\{C L^{*}(t-1, O N), C L^{*}(t-1, O F F)+S U(i, t)\right\}+L(t, O N)$

$C L^{*}(t, O F F)=\min \left\{C L^{*}(t-1, O N)+S D(i, t), C L^{*}(t-1, O F F)\right\}+L(t, O F F)$ 


\section{Chapter 5}

\section{Simulations and Test Results}

This chapter presents all the results of this research by detailing the AEP feeder model conversion in a flow chart followed by the development and validation of distribution power flows for both feeders. PV is integrated into the developed feeder model at various locations and penetration levels and voltage and losses are plotted. Further, the impact of wind availability on the PBUC schedules of a Genco is explained in detail using simulated wind intermittency scenarios. Inferences are drawn from these results to support the proposed objectives and goals of this thesis work.

\subsection{Final Feeder Data Format in OpenDSS}

Individual distribution component models described in Chapter 4 are integrated to develop the final feeder model in OpenDSS. Figure 5-1 shows the model conversion strategy from CYMDIST to OpenDSS. The network information in CYMDIST is decoded into the required component file format in OpenDSS. The component development is followed by instantiating the circuit preliminaries namely the circuit definition, voltage source definition and the base voltage specification for the circuit. The connectivity and location of each component on the feeder is established by the -master file" based on the individual component files and the Geographical Information System (GIS) model. The master file is compiled and debugged to identify and remove scripting, syntax and logic issues. The circuit solution mode and power flow parameters like tolerance and number of iterations required are defined in the fun file" to carry out three phase unbalanced distribution power flow. The results of the distribution power flow namely node voltages, line currents, real and reactive power flows can be accessed by text based and graphical plot formats. These power flow results are used to validate the model conversion and further form the basis for performing PV integration studies, the results of which are discussed in subsections below. 


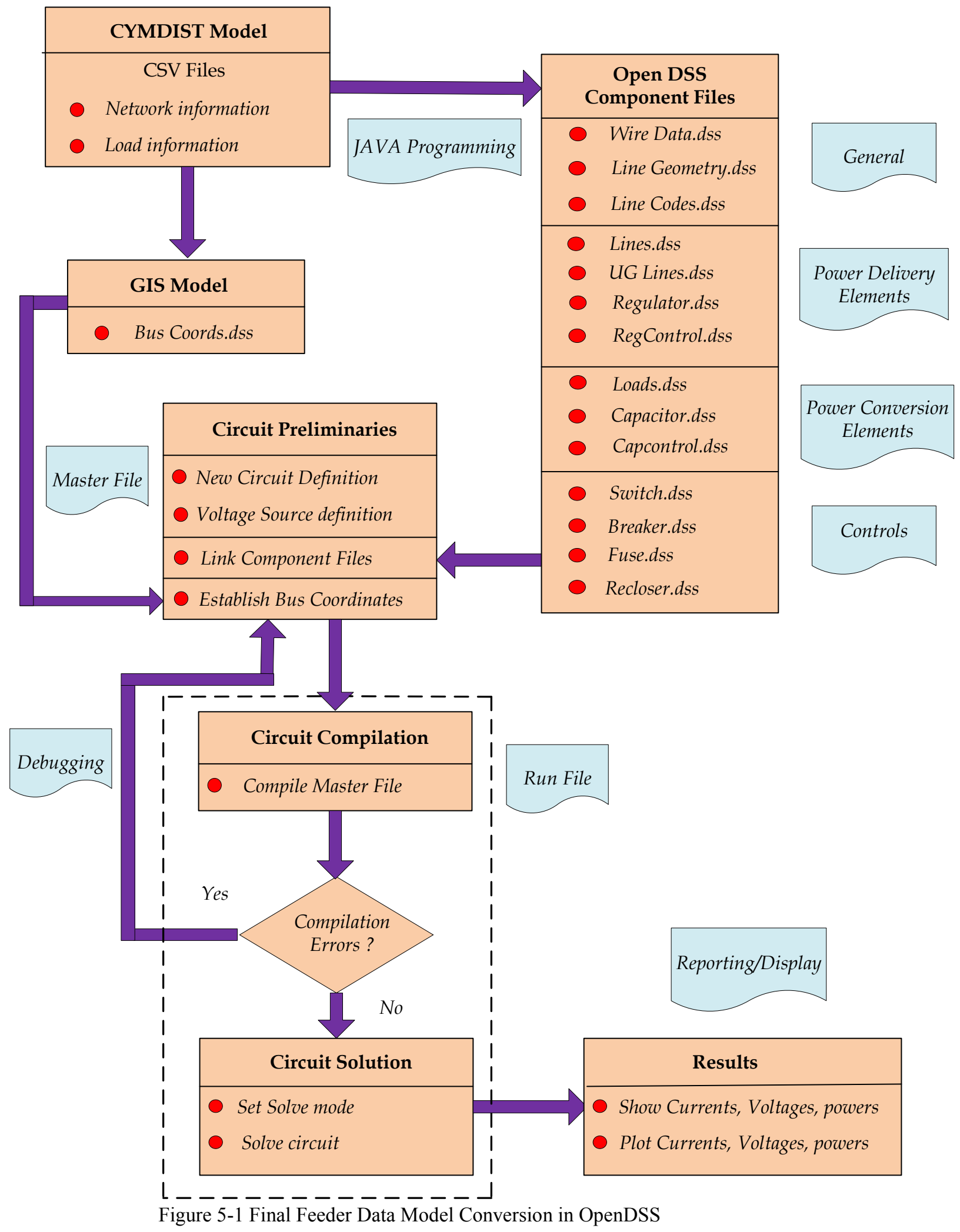




\subsection{Distribution Power Flow Results for Feeder 1 of AEP System}

Table 5-1 Summary of Power flow for Feeder 1

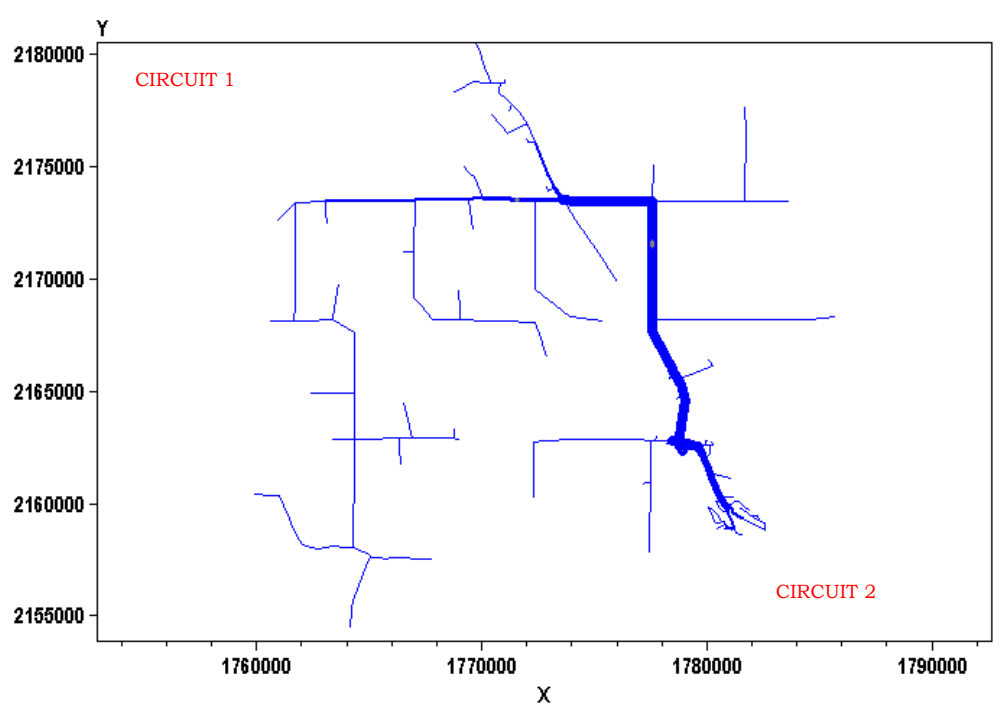

\begin{tabular}{|l|l|}
\hline Status & SOLVED \\
\hline Solution Mode & Direct \\
\hline Load multiplier & 1.00 \\
\hline Devices & 640 \\
\hline Buses & 395 \\
\hline Nodes & 650 \\
\hline Max p.u. voltage & 1.0375 V p.u. \\
\hline Min p.u. voltage & 0.99984 V p.u. \\
\hline Total Active power & 2.04259 MW \\
\hline Total reactive power & $1.00041 \mathrm{MVAR}$ \\
\hline Total Active losses & $0.031 \mathrm{MW}(1.519 \%)$ \\
\hline Total Reactive losses & $0.0078 \mathrm{MVAR}$ \\
\hline Frequency & $60 \mathrm{~Hz}$ \\
\hline
\end{tabular}

Figure 5-2 OpenDSS power flow plot for Feeder 1

A distribution power flow was run for feeder 1 of the AEP system and Figure 5-2 shows a one line diagram of feeder 1 modeled in OpenDSS. Table 5-1 summarizes the distribution power flow results for feeder 1. The important aspects of the distribution power flow for feeder 1 are highlighted below:

- The considered Feeder 1 is radial and supplied by a $12.47 \mathrm{KV}$ medium voltage substation modeled as an infinite source.

- The distribution system includes two main circuits with laterals and distributed loads.

- All the load buses in this system are modeled as PQ type loads.

- The system base is $100 \mathrm{MVA}$ and $12.47 \mathrm{KV}$.

- Three voltage regulators (two 3-phases and one single phase) are employed in this feeder, one in the substation and the other two at the middle and far ends of the feeder.

- The aggregated loads represent a mixture of residential and industrial loads and the total load on the system is 2.27 MVA (2.042 MW and 1.00 MVAR) and the active power losses represent 1.52 $\%$ of the total system load.

- The node voltages of feeder 1 are in the range of $0.99 \mathrm{~V}$ p.u. and $1.037 \mathrm{~V}$. p.u. 


\subsection{Distribution Power Flow results for Feeder 2 of AEP System}

Table 5-2 Summary of Power flow for Feeder 2

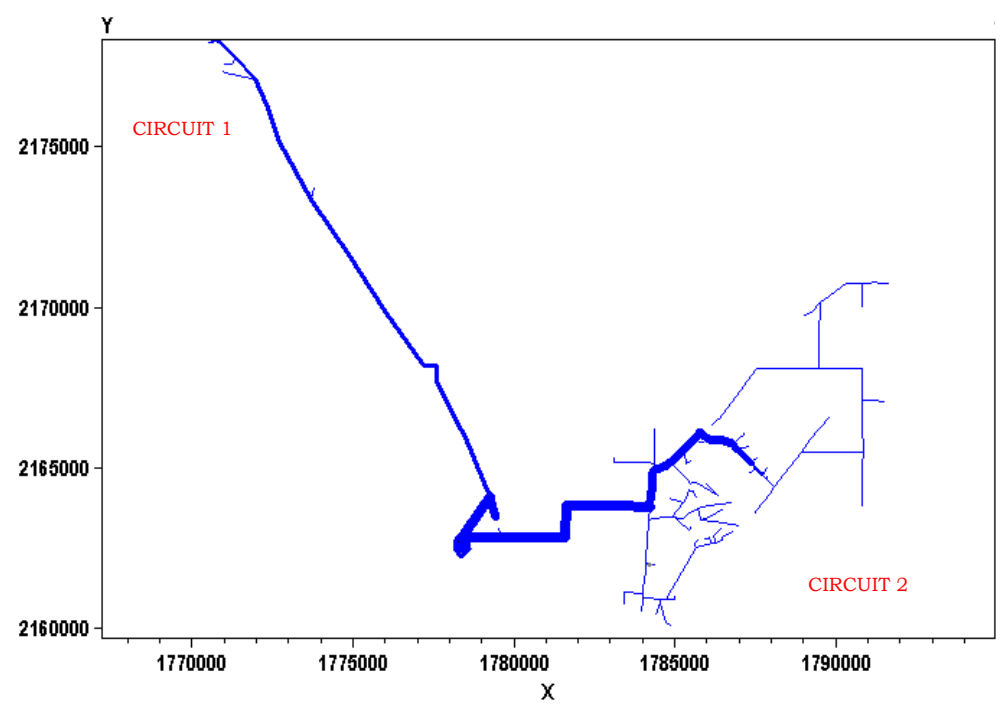

\begin{tabular}{|l|l|}
\hline Status & SOLVED \\
\hline Solution Mode & Direct \\
\hline Load multiplier & 1.00 \\
\hline Devices & 560 \\
\hline Buses & 266 \\
\hline Nodes & 566 \\
\hline Max p.u. voltage & $1.03 \mathrm{~V}$ p.u. \\
\hline Min p.u. voltage & $1.0033 \mathrm{~V}$ p.u. \\
\hline Total Active power & $12.994 \mathrm{MW}$ \\
\hline Total reactive power & $2.07443 \mathrm{MVAR}$ \\
\hline Total Active losses & $0.1249 \mathrm{MW}(0.9618 \%)$ \\
\hline Total Reactive losses & $0.0323407 \mathrm{MVAR}$ \\
\hline Frequency & $60 \mathrm{~Hz}$ \\
\hline
\end{tabular}

Figure 5-3 OpenDSS power flow plot for Feeder 2

A distribution power flow was run for feeder 2 of the AEP system and Figure 5-3 shows a one line diagram of feeder 2 modeled in OpenDSS. Table 5-2 summarizes the distribution power flow results for feeder 2. The important aspects of the distribution power flow for feeder 2 are highlighted below:

- The considered feeder 2 is radial and supplied by a $12.47 \mathrm{KV}$ medium voltage substation modeled as an infinite source.

- The distribution system includes two main circuits with laterals and distributed loads.

- All the load buses in this system are modeled as PQ type loads.

- The system base is $100 \mathrm{MVA}$ and $12.47 \mathrm{KV}$.

- A single 3-phase voltage regulator is employed in this feeder at the substation.

- Switched capacitor banks are present at seven different locations along the feeder.

- The aggregated loads represent a mixture of residential and industrial loads and the total load on the system is 13.15 MVA (12.994 MW and 2.074 MVAR) and the active power losses represent $0.96 \%$ of the total system load.

- The node voltages of feeder 2 are in the range of 1.003 V. p.u. and 1.03 V. p.u. 


\subsection{Validation of Power Flow Results of AEP System}

Subsequent to obtaining the distribution power flow results for the feeders they were validated with available results in CYMDIST. The section currents, node voltages and the real and reactive power flows through a section are the parameters chosen for the comparative study. The maximum and average error for these system parameters when compared with available CYMDIST results are tabulated in 5-3.

Table 5-3 Maximum and average errors for conversion model from CYMDIST to OpenDSS.

\begin{tabular}{|c|c|c|c|c|}
\hline \multirow[t]{2}{*}{ Parameter } & \multicolumn{2}{|c|}{ Feeder 1} & \multicolumn{2}{|c|}{ Feeder 2} \\
\hline & $\begin{array}{l}\text { Maximum } \\
\text { Error }(\%)\end{array}$ & $\begin{array}{c}\text { Average Error } \\
(\%)\end{array}$ & $\begin{array}{l}\text { Maximum } \\
\text { Error }(\%)\end{array}$ & $\begin{array}{c}\text { Average Error } \\
(\%)\end{array}$ \\
\hline Current $I_{A}(A)$ & 1.948 & 0.46 & 2.485 & 0.800 \\
\hline Current $I_{B}(A)$ & 2.0 & 0.429 & 2.5 & 0.924 \\
\hline Current $I_{C}(A)$ & 1.867 & 0.5 & 2.491 & 0.915 \\
\hline Real Power $\mathrm{KW}_{\mathrm{A}}$ & 2.792 & 0.799 & 2.7 & 0.779 \\
\hline Real Power $K_{W_{B}}$ & 2.579 & 0.787 & 2.865 & 0.871 \\
\hline Real Power $\mathrm{KW}_{\mathrm{C}}$ & 2.619 & 0.714 & 2.87 & 0.916 \\
\hline Reactive power $\mathrm{KVAR}_{\mathrm{A}}$ & 2.593 & 0.611 & 2.93 & 1.01 \\
\hline Reactive power $\mathrm{KVAR}_{\mathrm{B}}$ & 2.677 & 0.683 & 2.914 & 1.06 \\
\hline Reactive power $\mathrm{KVAR}_{\mathrm{C}}$ & 2.755 & 0.946 & 2.89 & 0.926 \\
\hline $\mathrm{KVA}_{\mathbf{A}}$ & 2.409 & 0.764 & 2.67 & 0.830 \\
\hline $\mathbf{K V A}_{\mathbf{B}}$ & 2.569 & 0.74 & 2.647 & 0.882 \\
\hline $\mathbf{K V A}_{\mathbf{C}}$ & 2.509 & 0.745 & 2.57 & 0.912 \\
\hline Voltage $V_{A}(V)$ & 0.684 & 0.219 & 0.352 & 0.102 \\
\hline Voltage $V_{B}(V)$ & 0.629 & 0.261 & 0.468 & 0.176 \\
\hline Voltage $V_{C}(V)$ & 0.763 & 0.386 & 0.301 & 0.451 \\
\hline
\end{tabular}

A systematic and thorough analysis of these errors leads to important inferences, namely:

i. The maximum and average error in node voltages for feeders 1 and 2 is less than $1 \%$.

ii. The average error in section currents for feeders 1 and 2 is less than $1 \%$, while the maximum error ranges from $1.8-2 \%$ for feeder 1 and $2.4-2.5 \%$.for feeder 2 .

iii. For feeder 1, maximum error in the real and reactive power ranges from $2.5-2.7 \%$ and the average error ranges from $0.6-0.9 \%$. For feeder 2, maximum error in the real and reactive power ranges from $2.7-2.9 \%$ and the average error ranges from $0.7-1.06 \%$.

iv. The error of each parameter was seen to change with different models used for distributed load. One third model for distributed load produced the largest error followed by the exact model and then the midpoint model for the distributed load. 
v. The circuit component models that greatly affect the errors are:

a) Overhead Line: It is observed that the errors were partly due to differences in susceptances of overhead line in OpenDSS compared to those available in CYMDIST.

b) Regulator: The errors were also due to the regulator modeled as low impedance, low loss transformer in OpenDSS which does not replicate a line drop compensator as in CYMDIST and this introduces errors in section power flows.

vi. The model conversion accuracy is seen by the less than $3 \%$ error in the results.

Figures 5-4(a) and (b) show a graphical representation three phase currents and node voltages of feeder 1 in CYMDIST and OpenDSS. Figures 5-5(a) and (b) show a graphical representation of three phase real and reactive powers in CYMDIST and OpenDSS.
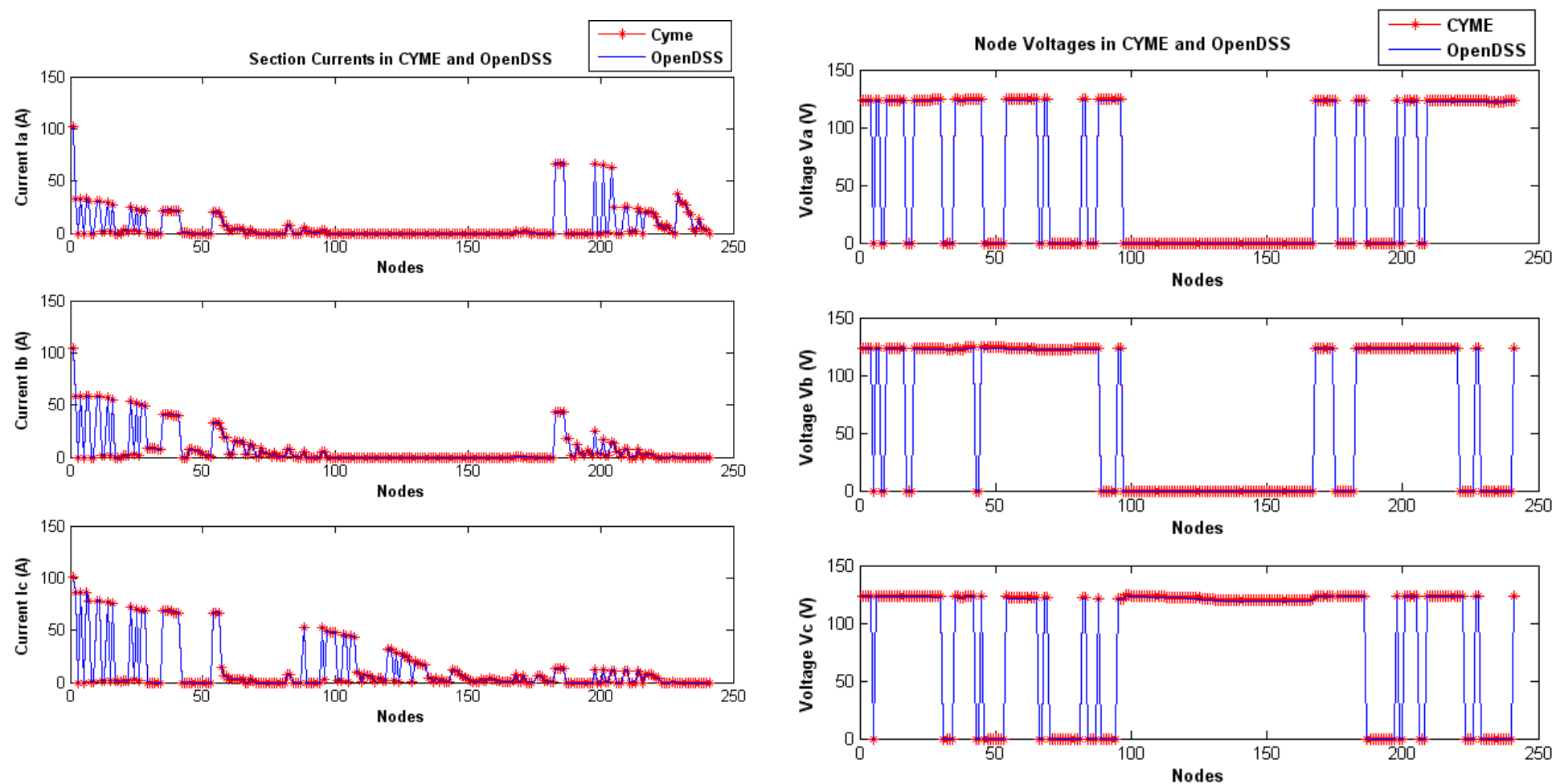

Figure 5-4 (a)-(b) Section Currents and Node Voltages in CYMDIST and OpenDSS 

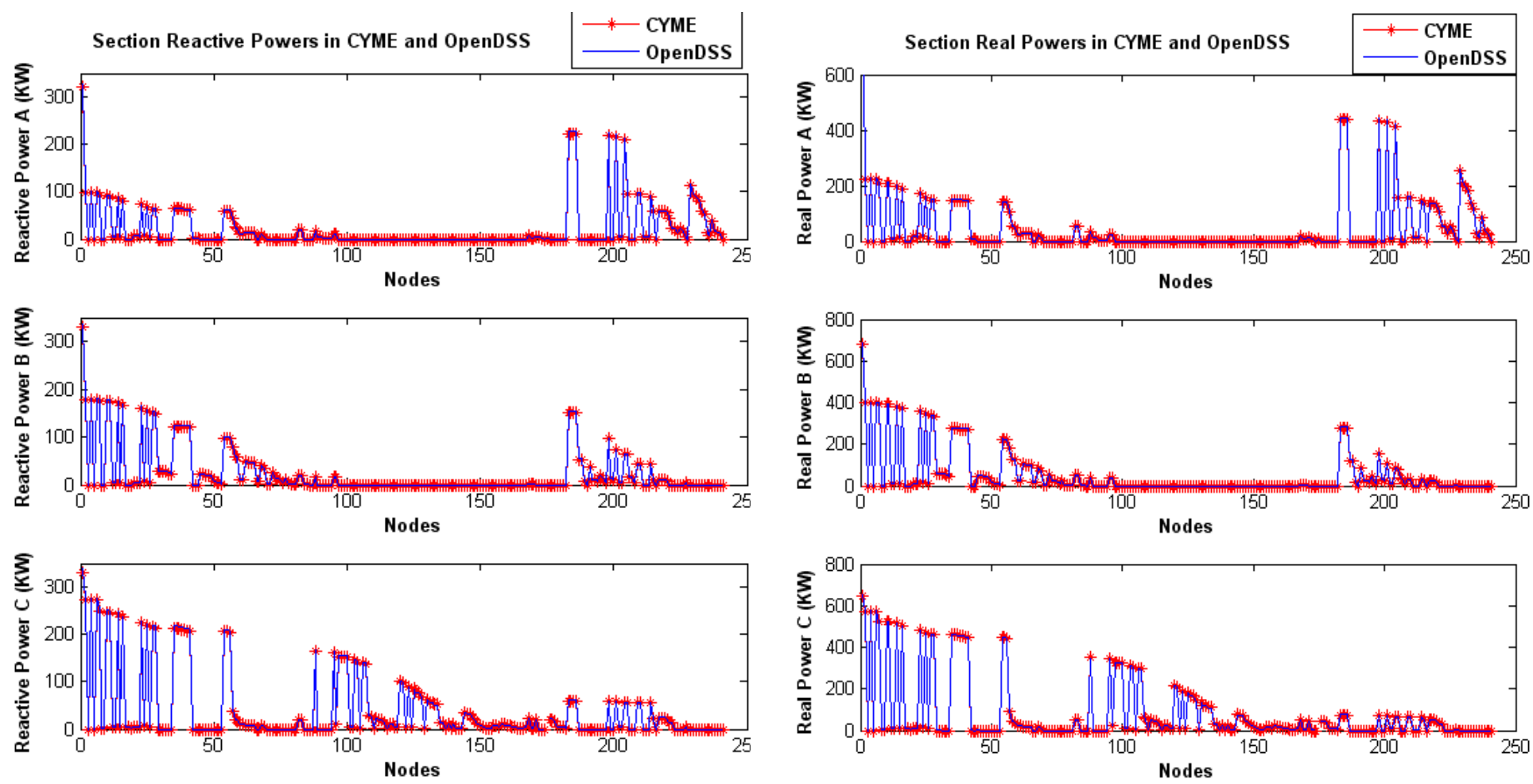

Figure 5-5 (a)-(b) Real and Reactive powers through a section in CYMDIST and OpenDSS

\subsection{Integration of PV with IEEE 13 node system}

PV integration studies were followed after verification of power flow results. The integration study was first done on the IEEE 13 node feeder, shown in Figure 5-6, prior to the analysis on the utility feeder model. The IEEE 13 node feeder is relatively small benchmark distribution feeder and was suitable to analyze the impacts of a small PV system on the distribution grid. Real time data for this study was obtained from the DTE Solar Currents \#2 PV facility in Southfield, Michigan and the PV facility in Southern California (Fountain Valley \# 1). The voluminous amount of data for the past few years was segregated, and parameters of interest namely average insolation in $\left(\mathrm{kWh} / \mathrm{m}^{2}\right)$ and the system efficiency (\%) is calculated. The monthly average insolation is calculated based on the daily insolation data. The system efficiency is calculated as the ratio of effective power output to the insolation received. It is noticed that the insolation patterns remain similar over the years, but vary with geographic locations. The system efficiency over the years is noticed to follow the same pattern, except for a slight decline in some cases. The system efficiency depends on a lot of factors like inverter operation and efficiency. Based on the insolation trends over the years from the data, typical insolation profiles are created and the peak insolation of $7 \mathrm{~kW} / \mathrm{m}^{2}$ is chosen for varying PV penetration levels from $10 \%$ to $50 \%$. 


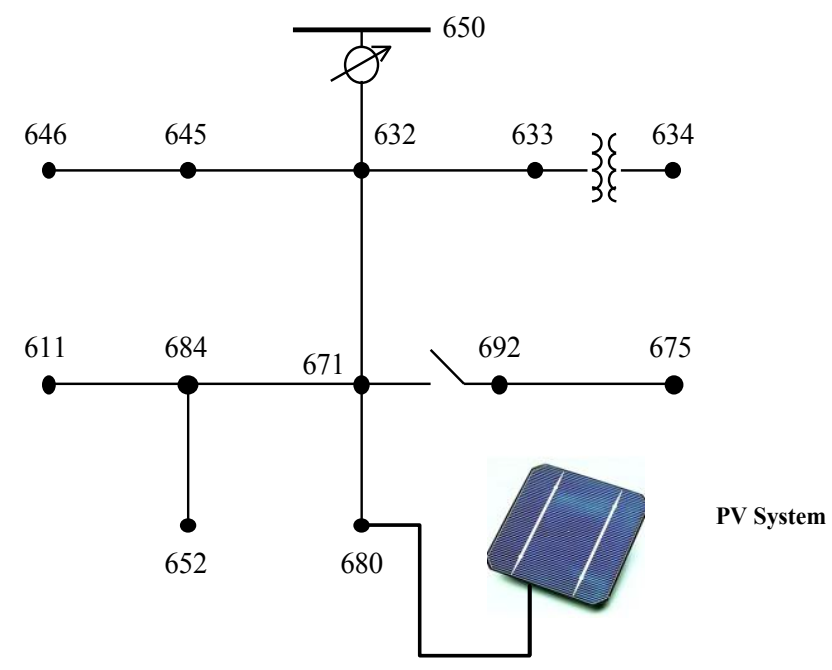

Figure 5-6 IEEE 13 Node test feeder with PV at end node

The PV system at node 680 modeled in MATLAB/Simulink as described in Chapter 4. The PV output corresponds to $320 \mathrm{~kW}$ at an insolation level of $7 \mathrm{~kW} / \mathrm{m}^{2}$. At this insolation level, each individual module produces a maximum power of $105 \mathrm{~W}$ as per our design specifications. Hence to achieve a power output of $320 \mathrm{~kW}, 3045$ individual PV modules are necessary. The penetration levels of the PV are increased to $1600 \mathrm{~kW}$ corresponding to $50 \%$ penetration. Table 5-4 shows the power flow results for system with $10 \%$ PV penetration.

Table 5-4 Voltage results of IEEE 13 node feeder with $10 \%$ PV penetration.

\begin{tabular}{|c|c|c|c|c|c|c|c|}
\hline Bus & $\begin{array}{c}\text { Va } \\
(\mathbf{k V})\end{array}$ & $\begin{array}{c}\text { Ang. } \\
(\mathbf{d e g})\end{array}$ & $\begin{array}{c}\text { Vb } \\
(\mathbf{k V})\end{array}$ & $\begin{array}{c}\text { Ang. } \\
(\mathbf{d e g})\end{array}$ & $\begin{array}{c}\mathbf{V c} \\
(\mathbf{k V})\end{array}$ & $\begin{array}{c}\text { Ang. } \\
(\mathbf{d e g})\end{array}$ & $\begin{array}{c}\text { Base } \\
\text { KV }\end{array}$ \\
\hline Source & 66.388 & 30 & 66.389 & -90 & 66.38 & 150 & 115 \\
\hline $\mathbf{6 5 0}$ & 2.4014 & 0 & 2.4015 & -120 & 2.401 & 120 & 4.16 \\
\hline RG 60 & 2.5212 & 0 & 2.4914 & -120 & 2.521 & 120 & 4.16 \\
\hline $\mathbf{6 3 3}$ & 2.4373 & -2.3 & 2.4367 & -120.7 & 2.417 & 118.3 & 0.48 \\
\hline $\mathbf{6 3 4}$ & 0.2745 & -3 & 0.2759 & -121.2 & 0.273 & 117.8 & 4.16 \\
\hline $\mathbf{6 7 1}$ & 2.3857 & -4.6 & 2.4447 & -120.3 & 2.342 & 117.1 & 4.16 \\
\hline $\mathbf{6 4 5}$ & & & 2.4194 & -120.8 & 2.418 & 118.3 & 4.16 \\
\hline $\mathbf{6 4 6}$ & & & 2.4153 & -120.9 & 2.413 & 118.4 & 4.16 \\
\hline $\mathbf{6 9 2}$ & 2.3857 & -4.6 & 2.4447 & -120.3 & 2.342 & 117.1 & 4.16 \\
\hline $\mathbf{6 7 5}$ & 2.3703 & -4.8 & 2.4501 & -120.5 & 2.338 & 117.1 & 4.16 \\
\hline $\mathbf{6 1 1}$ & & & & & 2.333 & 116.9 & 4.16 \\
\hline $\mathbf{6 5 2}$ & 2.3676 & -4.5 & & & & & 4.16 \\
\hline $\mathbf{6 7 0}$ & 2.4258 & -3 & 2.4404 & -120.5 & 2.393 & 117.8 & 4.16 \\
\hline $\mathbf{6 3 2}$ & 2.4445 & -2.3 & 2.4418 & -120.7 & 2.423 & 118.3 & 4.16 \\
\hline $\mathbf{6 8 0}$ & 2.3899 & -4.3 & 2.4502 & -120 & 2.344 & 117.4 & 4.16 \\
\hline $\mathbf{6 8 4}$ & 2.3811 & -4.6 & & & 2.338 & 117 & 4.16 \\
\hline
\end{tabular}



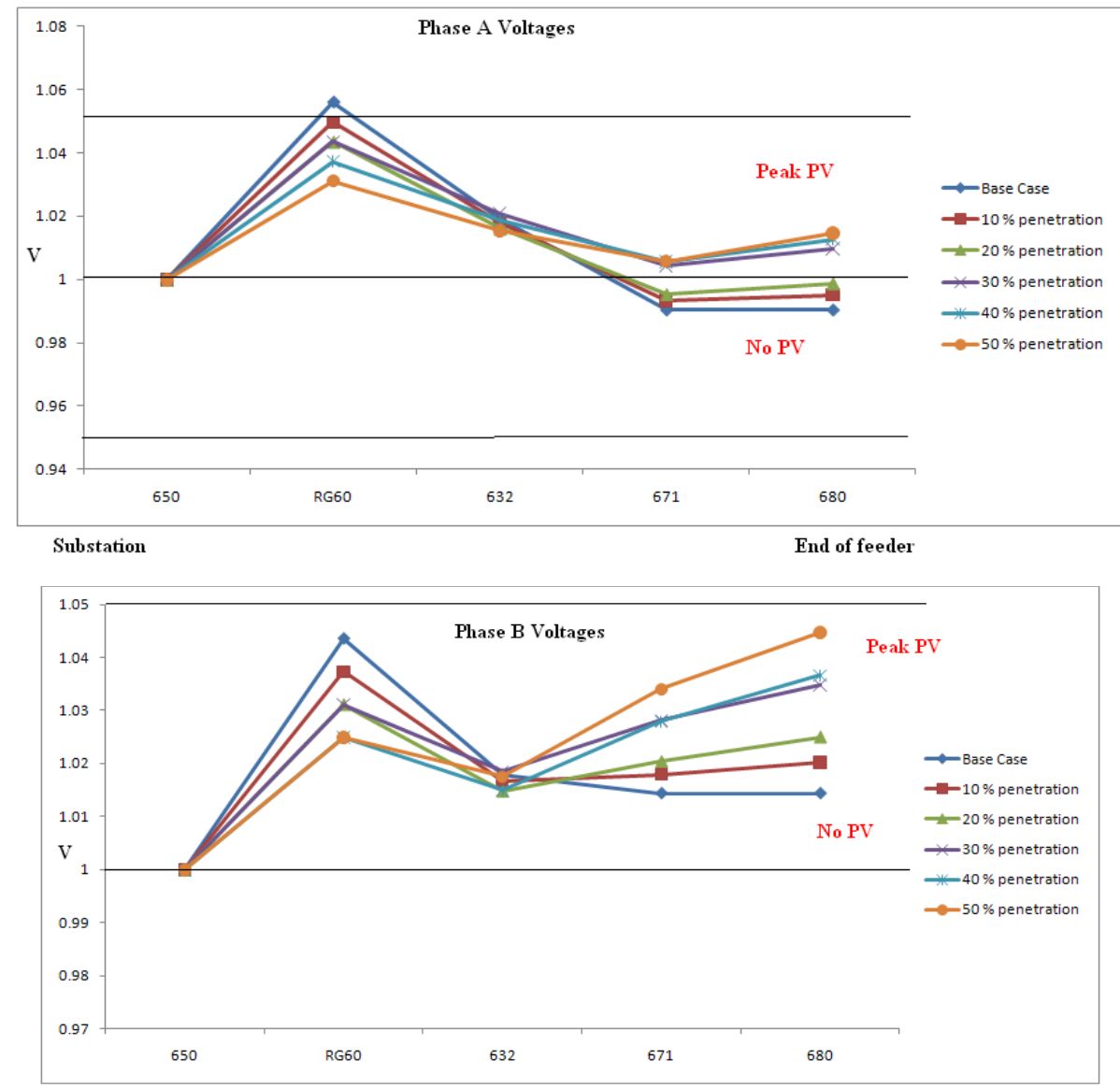

$\begin{array}{lll}\text { Substation } & \text { End of feeder }\end{array}$

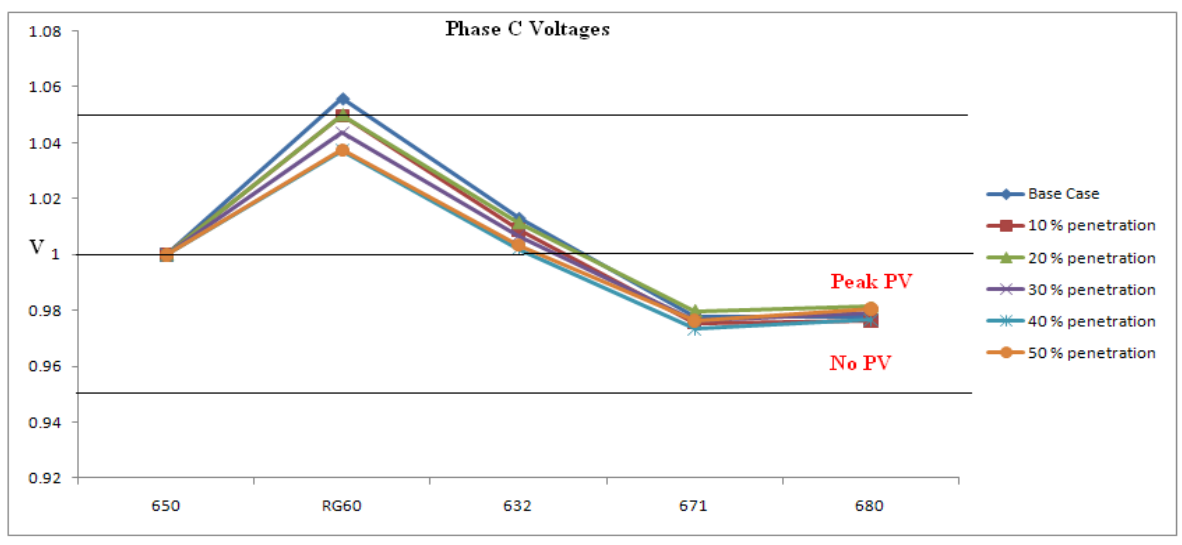

Substation

End of feeder

Figure 5-7 Phase Voltage variations of IEEE 13 node feeder with PV

Figure 5-7 shows the variation of voltages from the substation to the feeder at varying PV penetrations. It can be noticed from the graphs that the tail end of the regulation zone of the feeder is forced to a higher voltage due to a large PV system located near the end of the feeder. In the base case scenario and at lower penetration levels, the regulator voltage is at the required tap setting to maintain the 
node voltages within specified limits. But at peak PV penetration, the end node voltages are higher than the sources voltage levels. This leads to a reverse power flow on the feeder and voltage unbalance.

The PV integration study on a smaller distribution system yielded important considerations for the studies on the utility feeder model.

- This study considered a snapshot power flow solution with the PV at a single location. For an accurate analysis of the PV model, time varying PV profile and load are necessary. A time based power flow analysis helps to capture the worst case scenarios for peak load and peak PV instances.

- Similarly the location of the PV system on the feeder has an impact on the voltage profiles and losses. For the PV integration study on the utility feeder model the utility intended locations are considered at the feeder head and feeder tail. Varying penetration levels of PV from $10 \%$ to $50 \%$ were considered for the trends of voltage profiles and losses to assess the worst case scenarios.

\subsection{PV integration study on utility feeder model}

The developed utility feeder 2 model is chosen for the PV integration study. Figure 5-8 shows the AEP feeder with the PV and meter locations.

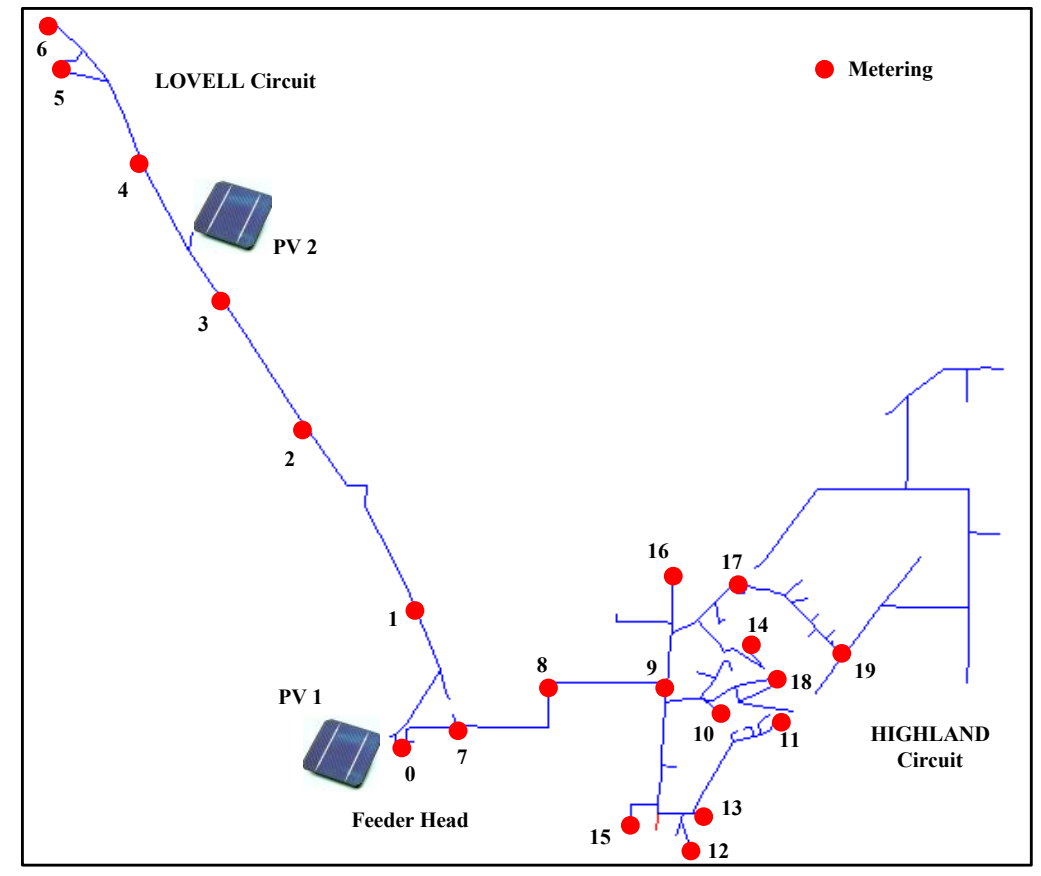

Figure 5-8 AEP Feeder model with PV and meter locations 
A typical daily load shape and solar generation shape is shown in Figure 5-9 and it can be observed that the peak load occurs at hour 18 whereas the peak PV generation occurs at around hour 12 in the afternoon. The time difference between the maximum PV system output and the residential demand peak results in a reduced local voltage regulation benefit to the system.

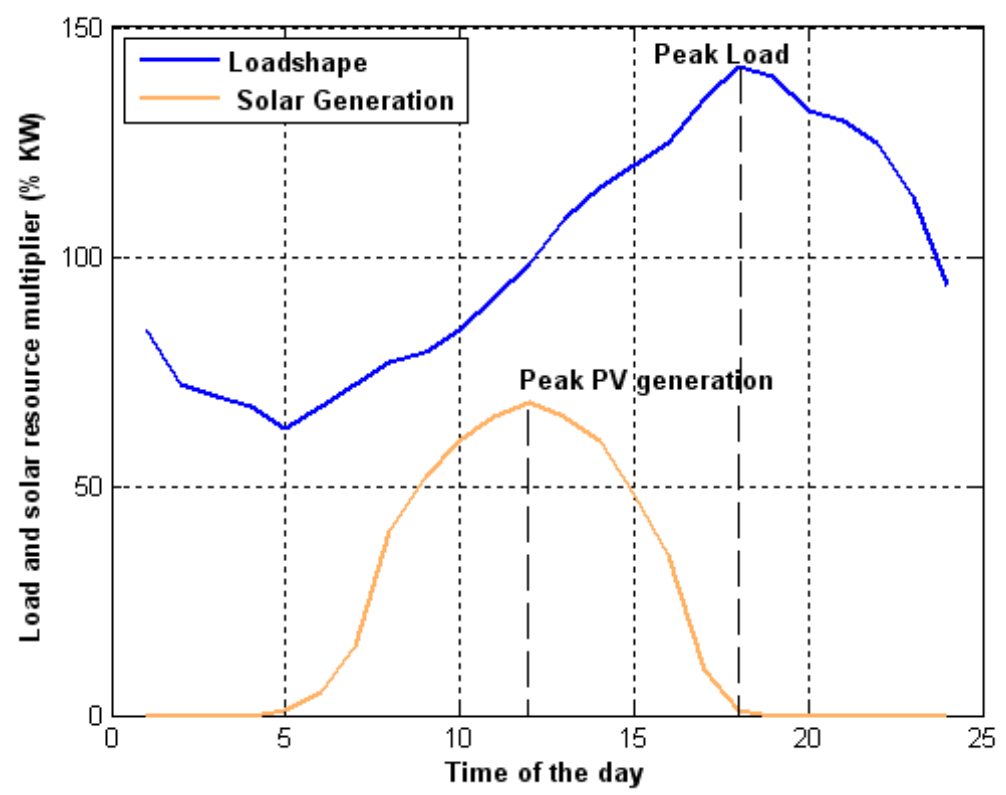

Figure 5-9 Time Varying PV and load profiles

The locations indicated in Figure 5-8, namely PV1 and PV2 are chosen for the study with different penetration levels as detailed in Table I.

Table 5-5 Solar Location and Penetration levels

\begin{tabular}{|l|l|l|l|l|}
\hline Scenario & Locations & \multicolumn{3}{|l|}{$\begin{array}{l}\text { Penetration levels } \\
\text { (MW) }\end{array}$} \\
\cline { 3 - 5 } & & $\mathbf{1 0} \%$ & $\mathbf{3 0} \%$ & $\mathbf{5 0} \%$ \\
\hline $\begin{array}{l}\text { Utility intended } \\
\text { Locations }\end{array}$ & PV 1 and PV 2 & 1.5 & 4.0 & 6.5 \\
\hline
\end{tabular}




\subsubsection{Voltage Profiles for Different Penetration Levels}

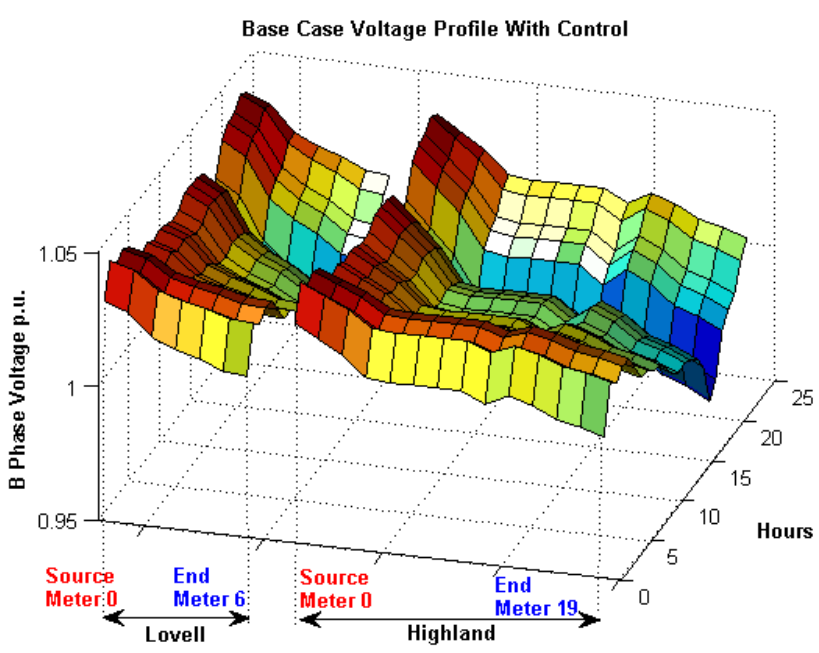

(a)

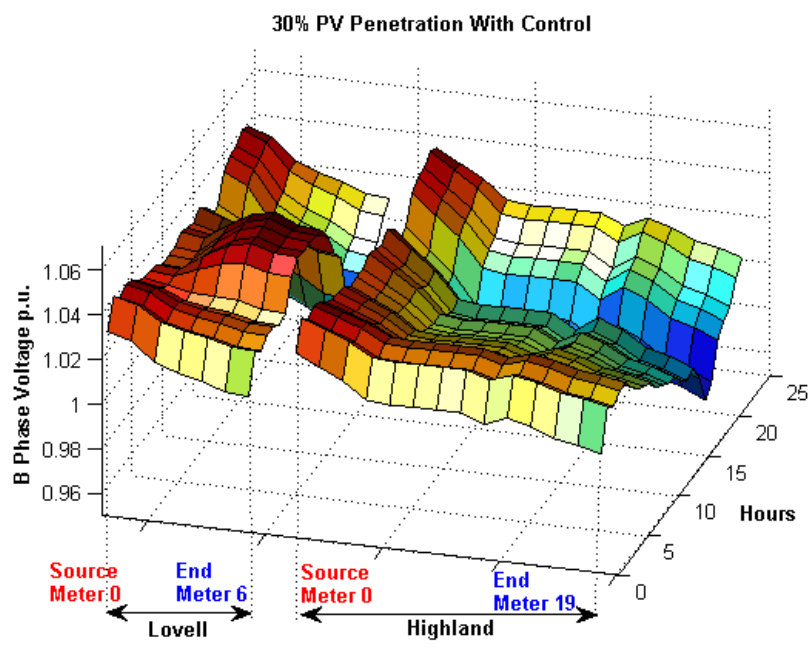

(c)

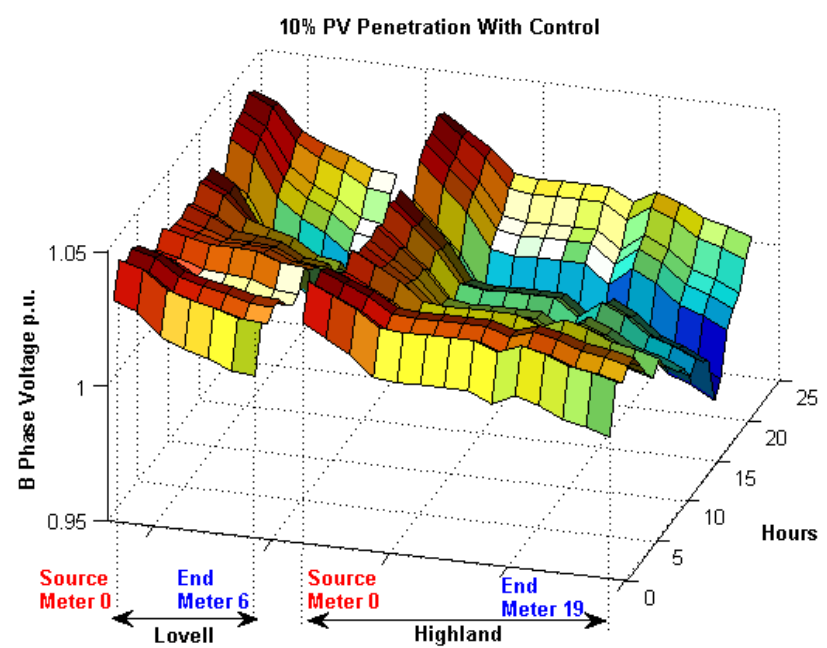

(b)

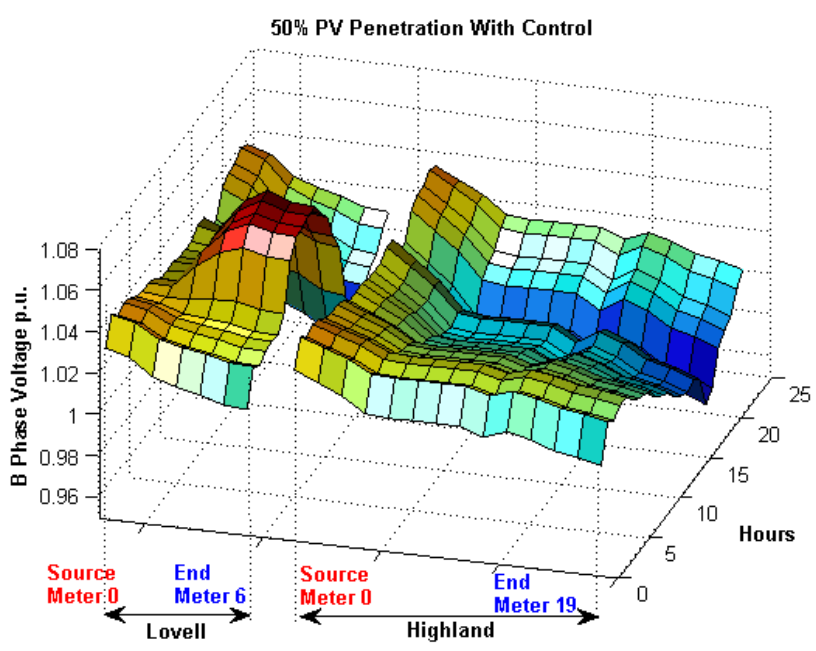

(d)

Fig. 5-10: Voltage profile of the circuit with controls (a): Base case (b) 10\% PV penetration (c) 30\% PV penetration (d) $50 \% \mathrm{PV}$ penetration

A 24 hour time series power flow is executed in OpenDSS with varying loads and PV generation. The control settings of the system are enabled which allows the regulators to change their tap settings to maintain system node voltages within limits. Figures 5-10(a) - (d) shows a comparison of the voltage profiles for varying PV penetration. The x-axis of Fig. 5-10 (a) - (d) represents the nodes from source to end of Lovell and Highland circuits, the y-axis of Fig. 5-10 (a) - (d) represent the hours of the day, and 
the z-axis of Fig. 5-10 (a) - (d) represents the B-phase voltages in p.u. Figure 5-10 (a) shows the base case solution with no PV generation present on the system indicating a wide range of voltages throughout the day owing to normal load variations. The surface plot of Figure 5-10 (a) indicates that there is no voltage deviation from limits of 0.95 p.u and 1.05 p.u for base case solution which is attributed to the regulator and capacitor control actions. Figure 5-10 (b) is obtained with a 10\% PV penetration on the system. Comparing surfaces of Figure 5-10 (a) and 5-10 (b) demonstrates an increase in voltages in the downstream nodes of Lovell circuit. However, this voltage deviation is maintained within limits by regulator and capacitor control actions. Figure 5-10 (c) is obtained with a 30\% PV penetration on the system. The same observations in voltage trends can be deduced for the surface plot of 5-10 (c). Figure 510 (d) is obtained with a $50 \%$ PV penetration on the system and demonstrates that as the penetration levels increase to $50 \%$, the voltage deviation in the downstream nodes of the Lovell circuit becomes considerable and cannot be mitigated with the existing regulator and capacitor controls. It can also be inferred from surface plots of 5-10(a) - 5-10 (d), that the voltage profiles of the Highland circuit remain unaffected due to absence of PV generators.

\subsubsection{Regulator Control Settings}

Based on the observations from the surface plots of 5-10 (b) - 5-10 (d), with an increase in PV penetration, there is an increase in voltage deviations in the downstream nodes of the Lovell circuit. At $50 \% \mathrm{PV}$ penetration, the existing regulator voltage set points fail to mitigate the voltage deviation. Hence, the voltage set point of the substation regulator must be lowered to maintain the voltage levels within limits. Figures 5-11 (a) and 5-11 (b) represent the B-phase voltages of the source with existing and lowered voltage setpoints for varying penetrations. Figures 5-11 (c) and 5-11 (d) represent the B-phase voltages of the end nodes in the system with existing and lowered voltage setpoints for varying penetrations. The effect of lowering the voltage setpoint results in a reduced base case voltage level at the source for the 24 hour period with an improvement in voltages for peak load periods from hours 17-19, which can be observed from Figures 5-11 (a) and 5-11 (b). Figures 5-11 (c) and 5-11 (d) clearly indicate that the voltages of the downstream nodes of Lovell circuit are maintained within limits with the lowered voltage setpoint. Figure 5-12 shows the tap changes for the three phases of the regulator during a 24-hour period for the mitigation of voltage violations. 


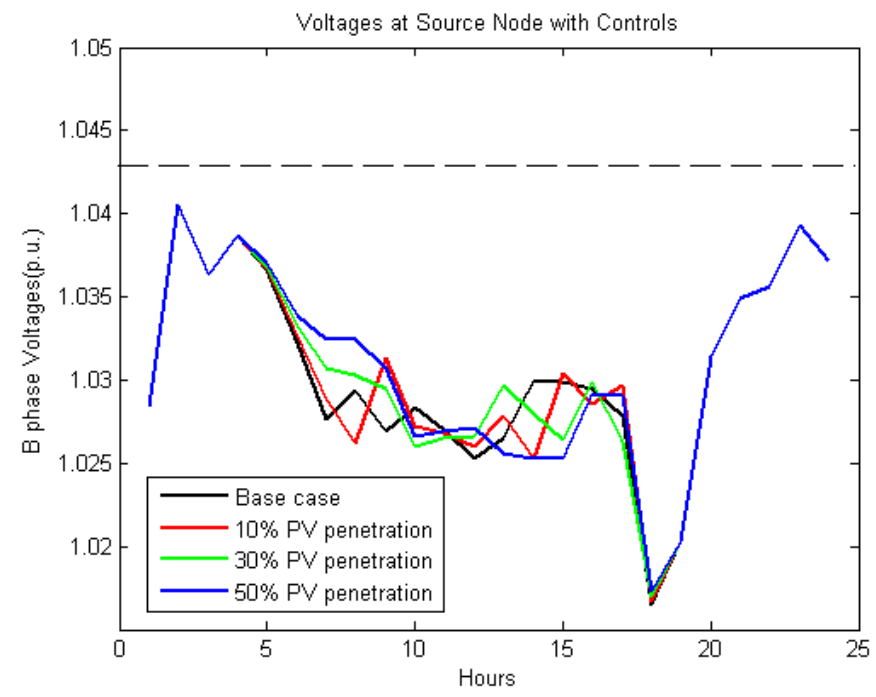

(a)

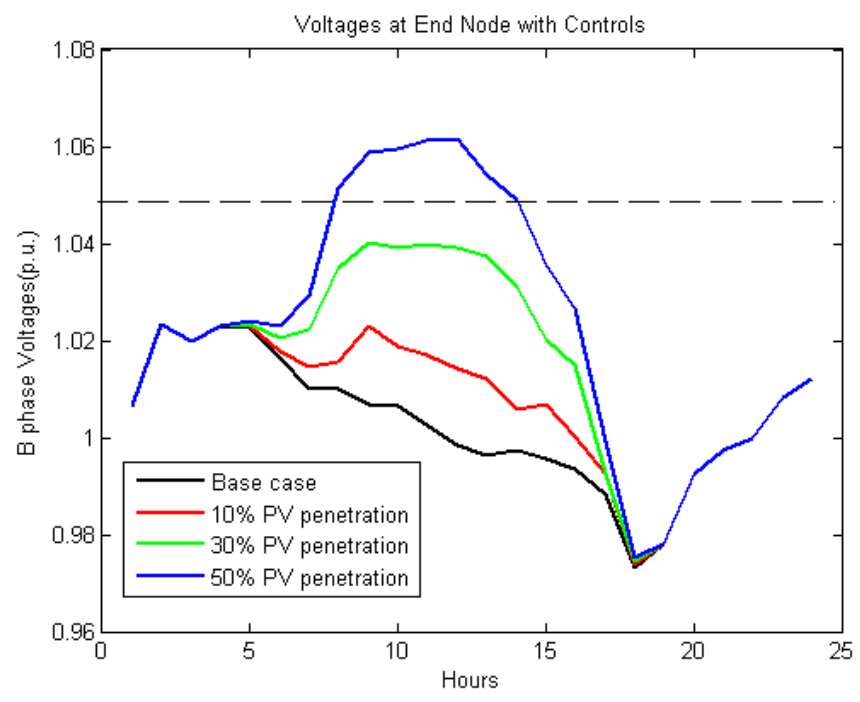

(c)

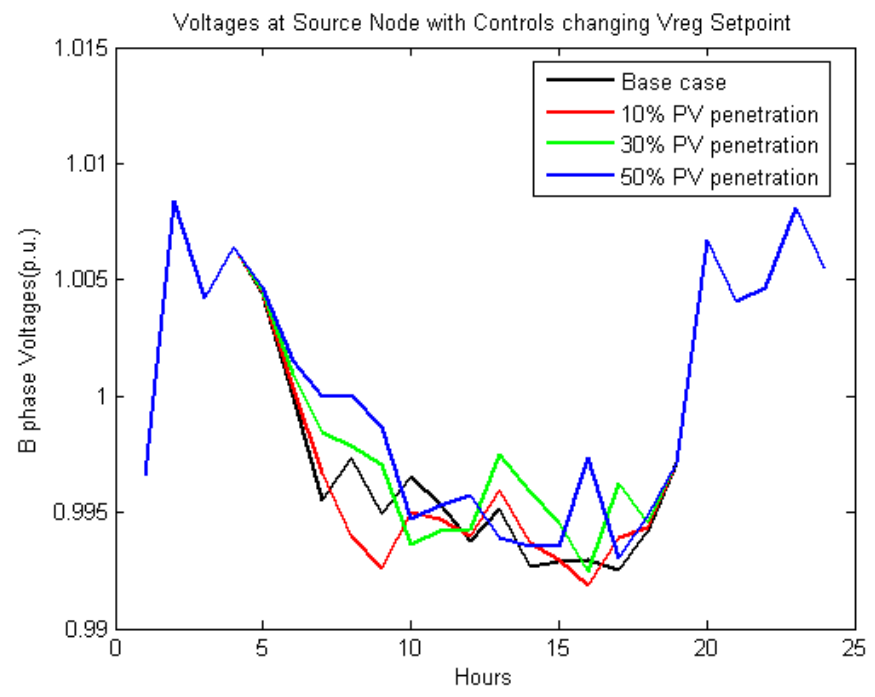

(b)

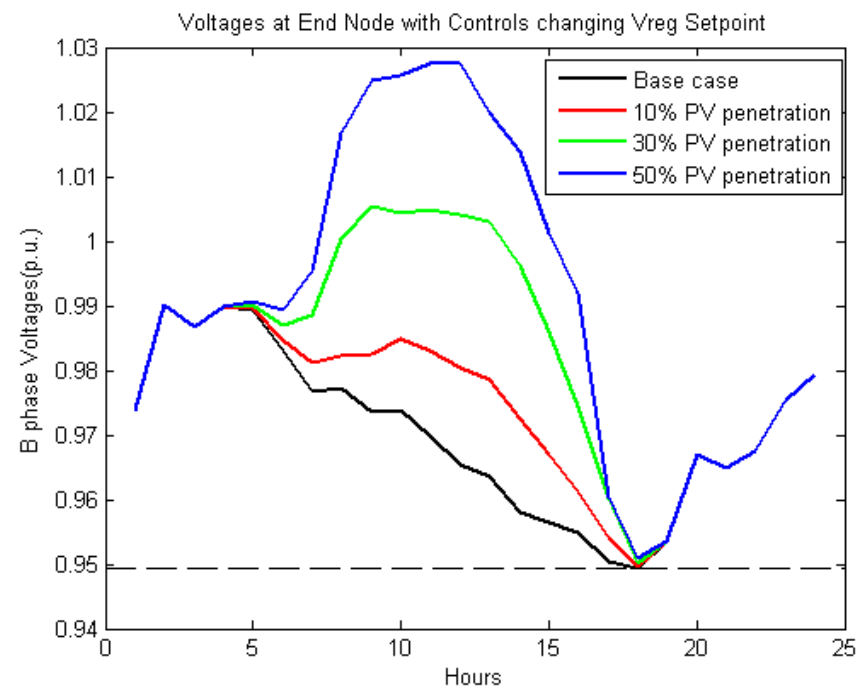

(d)

Fig. 5-11. (a) Source node voltages - Existing control settings $\quad$ (b) Source node voltages with lower voltage regulator set point (c) End node voltages- Existing control settings (d) End node voltages with lower voltage regulator set point 


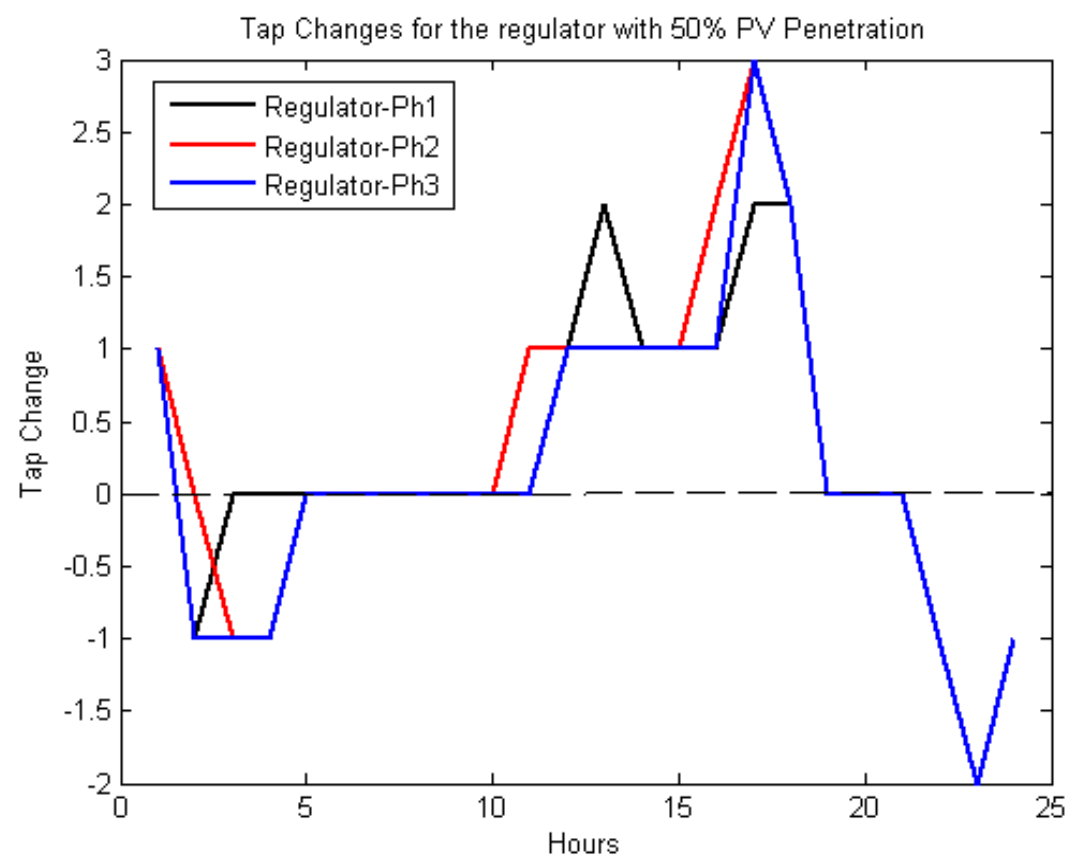

Figure 5-12: 24-hour Three phase Regulator tap changes

\subsubsection{Losses of the System}

The percentage losses of the system for the base case and varying PV penetration levels are shown in Figure 5-13. It is can be inferred that during hours 1-5 and hours 19-24 with low or non availability of PV power, the system losses remain unaffected and correspond to base case losses. During hours 6-18, a decrease in losses is observed for penetration levels of $10 \%$ and $30 \%$. However, as the PV penetration increases to $50 \%$, a marginal increase in the system losses is observed. The losses of the system depend on the location of the load with respect to the location of the PV generator. The increase in losses can be attributed to the specific feeder loading conditions. In this case, the heavily loaded source ends of the Lovell circuit are fed from PV at the downstream sections, results in increased losses. Another factor affecting the losses is the current carrying capacity of the conductors. In this case the source end of the feeder consists of conductors with larger current carrying capacity and downstream sections are designed with smaller current carrying capacity. An increased power flow in the downstream sections due to high PV penetration results in increased losses. 


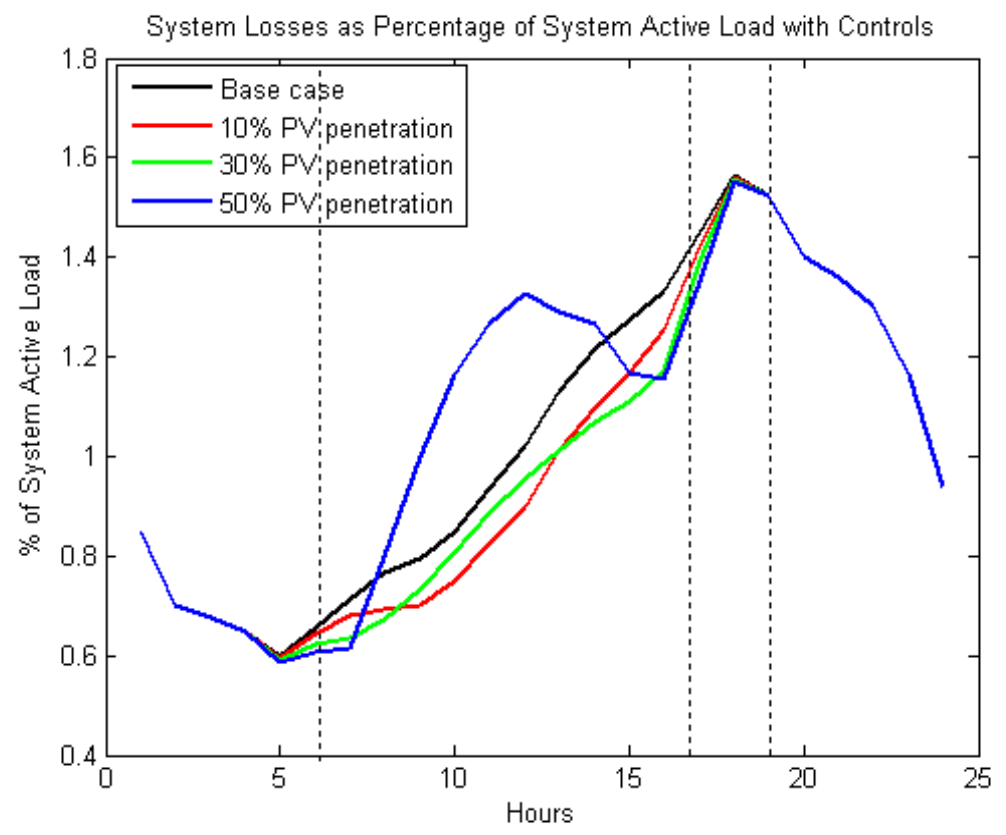

Fig. 5-13: Percentage System losses for various penetration levels

\subsubsection{Effect of Varying PV/ Cloud Cover}

To study the effect of varying PV insolation and cloud cover on the system, PV generation and loading are chosen at 10-minute intervals for a 24 hour period as shown in Figure 5-14. Figures 5-15 and 5-16 represent the source node and end node voltages of the Lovell circuit with $50 \%$ PV penetration under a cloud cover scenario.

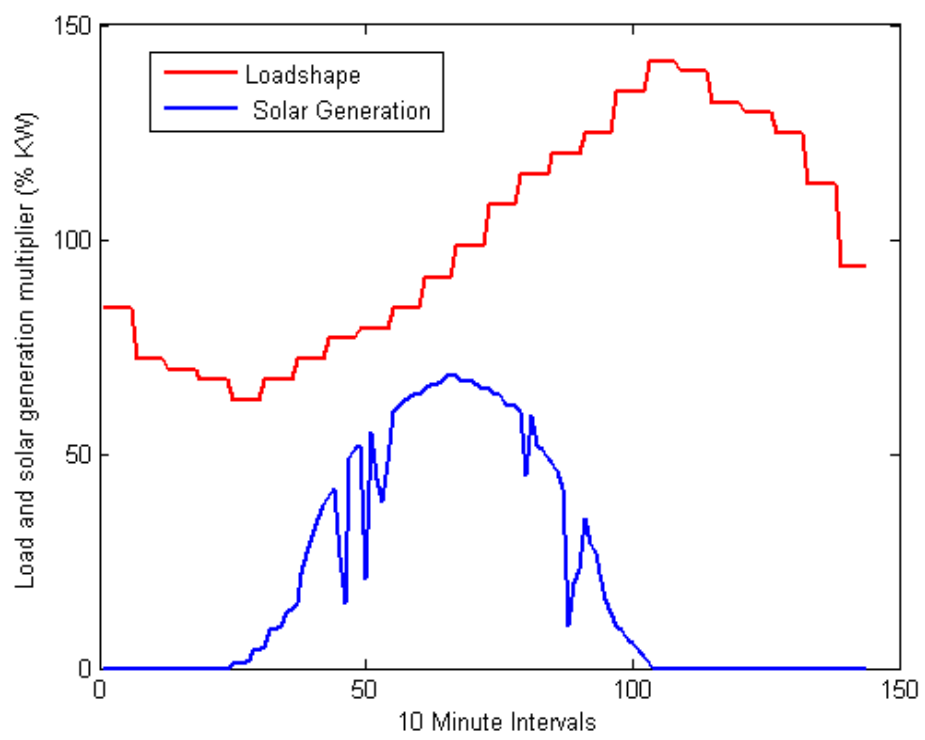

Fig. 5-14: 10-minute varying PV and load shapes to simulate cloud cover 


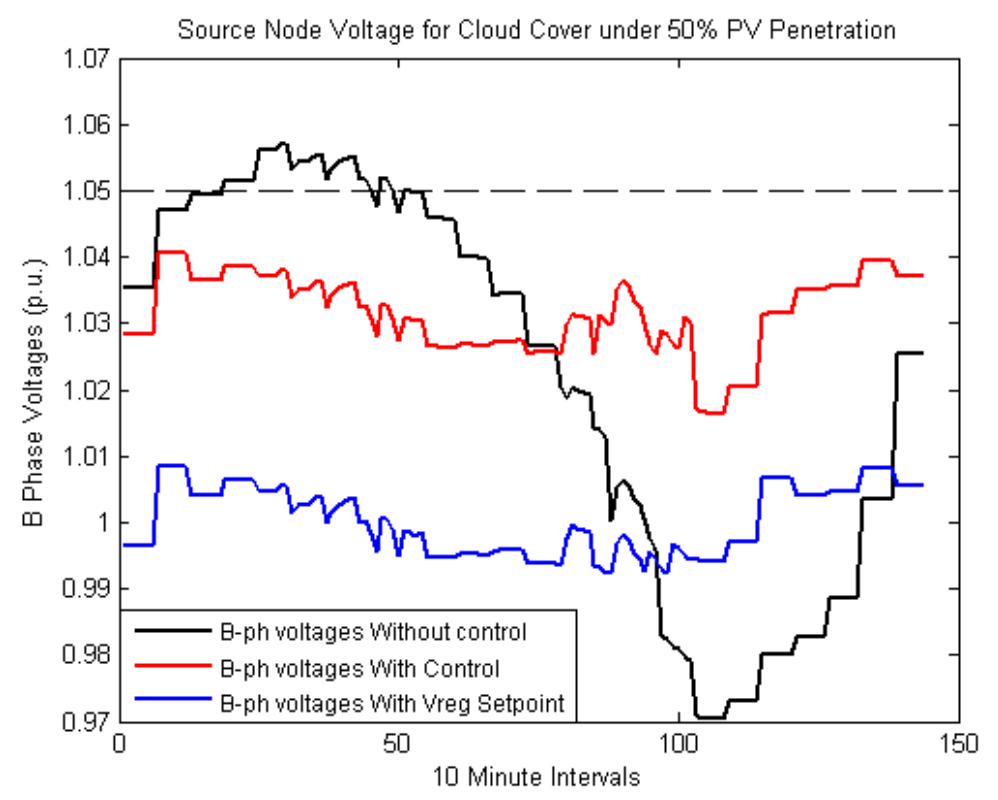

Fig. 5-15 Source node voltages with 50\% PV penetration under a cloud cover scenario

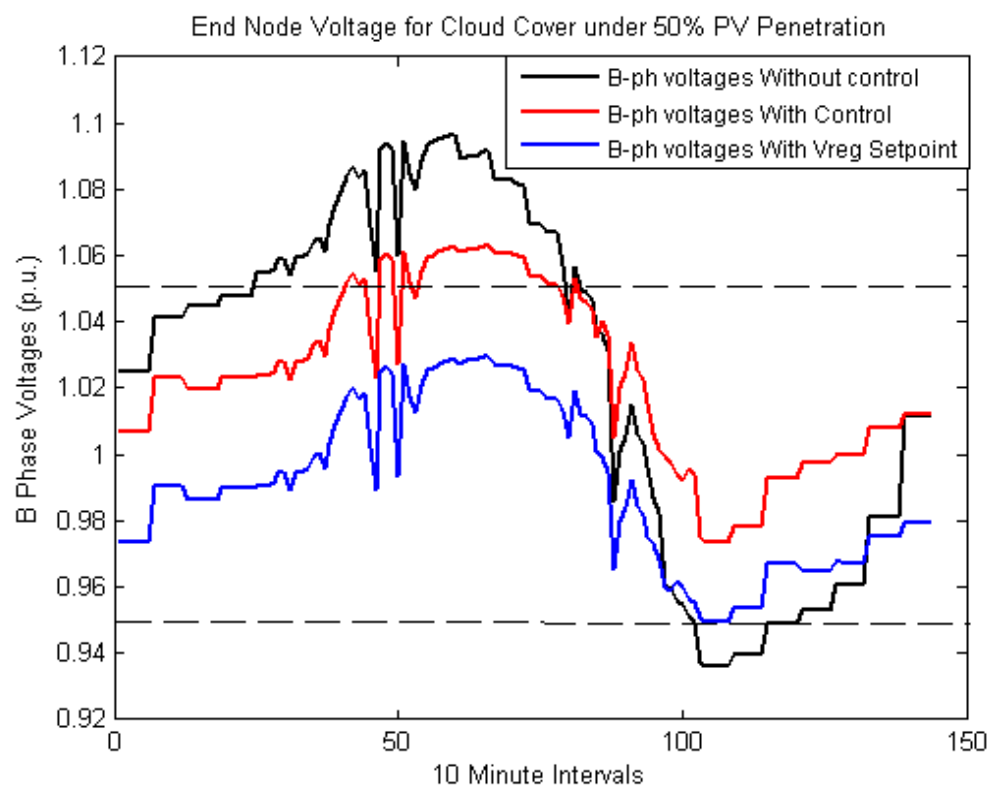

Fig. 5-16 End node voltages with 50\% PV penetration under a cloud cover scenario.

The voltage violations at the source node during lightly loaded hours 2-6 are mitigated by the control actions of regulators and capacitor banks and the voltages at heavily loaded hours 16-19 are maintained within limits as seen in Figure 5-15. Figure 5-16 shows that the voltages at the end node of the Lovell circuit under 50\% PV penetration are violated without regulator control and can be mitigated by regulator control with a lower voltage setpoint. 


\subsection{Impacts of DG on power market operation.}

To assess the impacts of DG on power market operations, a Genco owning distribution side windfarm and two non-wind generation units is simulated for a deregulated market operation. The test system used for this research consists of a 6-bus transmission system and a 3-bus distribution system as shown in Figure 5-17. The transmission system consists of buses 1-6, and two conventional synchronous generators are connected at buses 1 and 3 respectively. A wind farm with a capacity of $59.4 \mathrm{MW}$ is connected to Bus 8. The distribution system consisting of buses 7-9 is connected to the transmission system through a 100 MVA transformer. The bus/branch and generator data for the test system are listed in Tables 5-6 and 5-7 respectively.

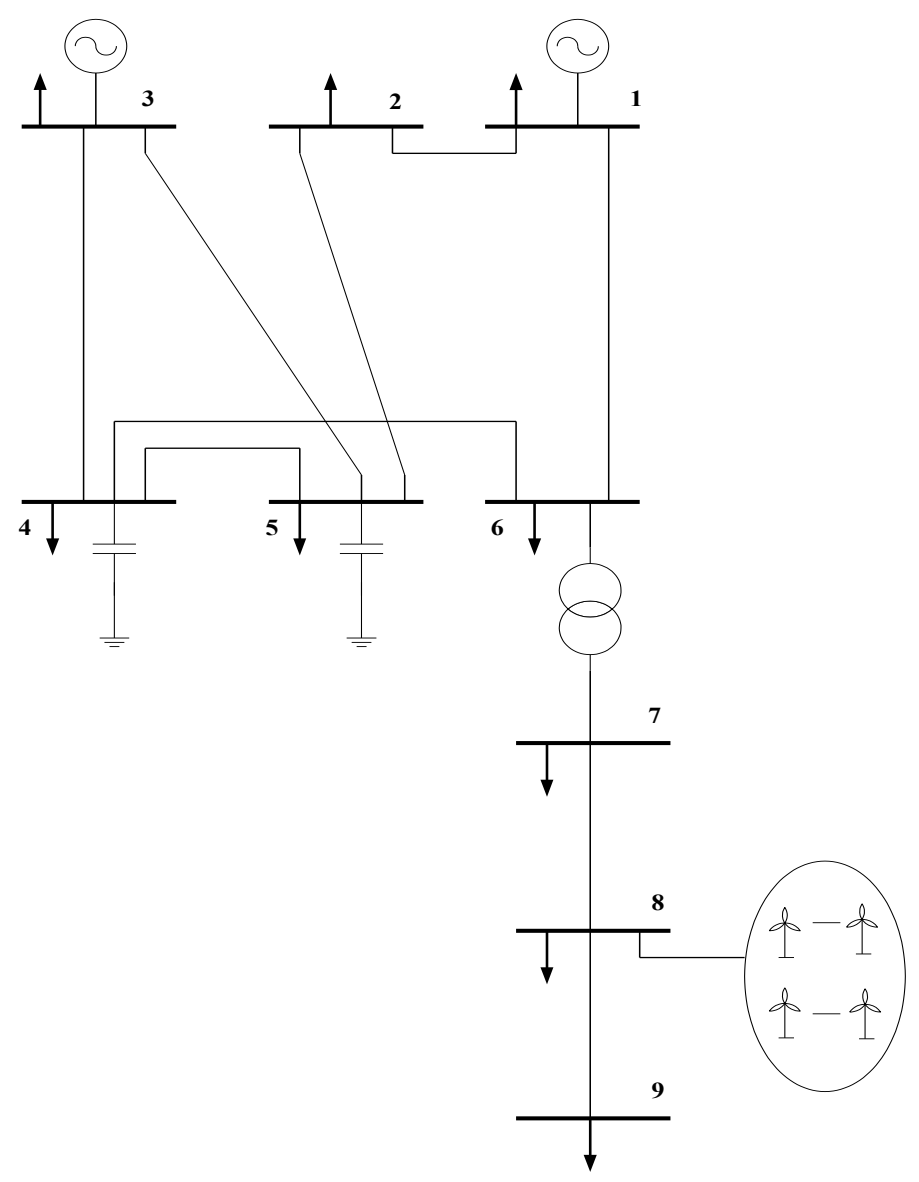

Figure 5-17: Nine bus test System 
Table 5-6 System Bus/Branch Data

\begin{tabular}{|c|c|c|c|c|c|c|}
\hline Bus & Pd (p.u.) & Qd (p.u.) & Lines & R (p.u.) & X (p.u.) & B(p.u.) \\
\hline 1 & 0.92 & 0.29 & $1-2$ & 0.1097 & 0.021 & 0.004 \\
\hline 2 & 0.78 & 0.39 & $1-6$ & 0.2732 & 0.0824 & 0.004 \\
\hline 3 & 0.73 & 0.19 & $2-5$ & 0.3185 & 0.107 & 0.005 \\
\hline 4 & 0.67 & 0.24 & $3-4$ & 0.2987 & 0.0945 & 0.005 \\
\hline 5 & 1.12 & 0.31 & $3-5$ & 0.1804 & 0.0662 & 0.003 \\
\hline 6 & 0.26 & 0.12 & $4-5$ & 0.1792 & 0.0639 & 0.001 \\
\hline 7 & 0.1 & 0.02 & $4-6$ & 0.098 & 0.034 & 0.004 \\
\hline 8 & 0.15 & 0.05 & $6-7$ & 0.1 & 0 & 0 \\
\hline 9 & 0.1 & 0.03 & $7-8$ & 0.082 & 0.054 & 0 \\
\hline & & & $8-9$ & 0.082 & 0.054 & 0 \\
\hline
\end{tabular}

Table 5-7 Generator Data

\begin{tabular}{|l|c|c|}
\hline \multicolumn{1}{|c|}{ Parameter } & Generator G1 & Generator G2 \\
\hline Unit Type & Coal & Oil \\
\hline Pmin (MW) & 20 & 100 \\
\hline Pmax(MW) & 250 & 500 \\
\hline Ramp Rate (MW/hrs) & 120 & 60 \\
\hline Quick Start (MW) & 5 & 20 \\
\hline Minimum ON time (hrs) & 2 & 2 \\
\hline Minimum OFF time( hrs) & 2 & 1 \\
\hline Initial State & ON & OFF \\
\hline Initial Hour (hrs) & 4 & 2 \\
\hline Fuel Price $(\$ / M B t u)$ & 2 & 2 \\
\hline Startup (MBtu) & 200 & 25 \\
\hline Cost Coefficient a (\$/MWh $\left.{ }^{2}\right)$ & 0.01 & 0.05 \\
\hline Cost Coefficient b $(\$ / M W h)$ & 25.5 & 8.5 \\
\hline Cost Coefficient c $(\$ / \mathrm{h})$ & 9 & 5 \\
\hline
\end{tabular}


The effect of intermittency and volatility of wind power and time varying loads for a 24 - hour period on the PBUC scehdule of a Genco was considered in this research work. Figure 5-18 shows predicted wind farm output for 24 hours considered in this thesis.

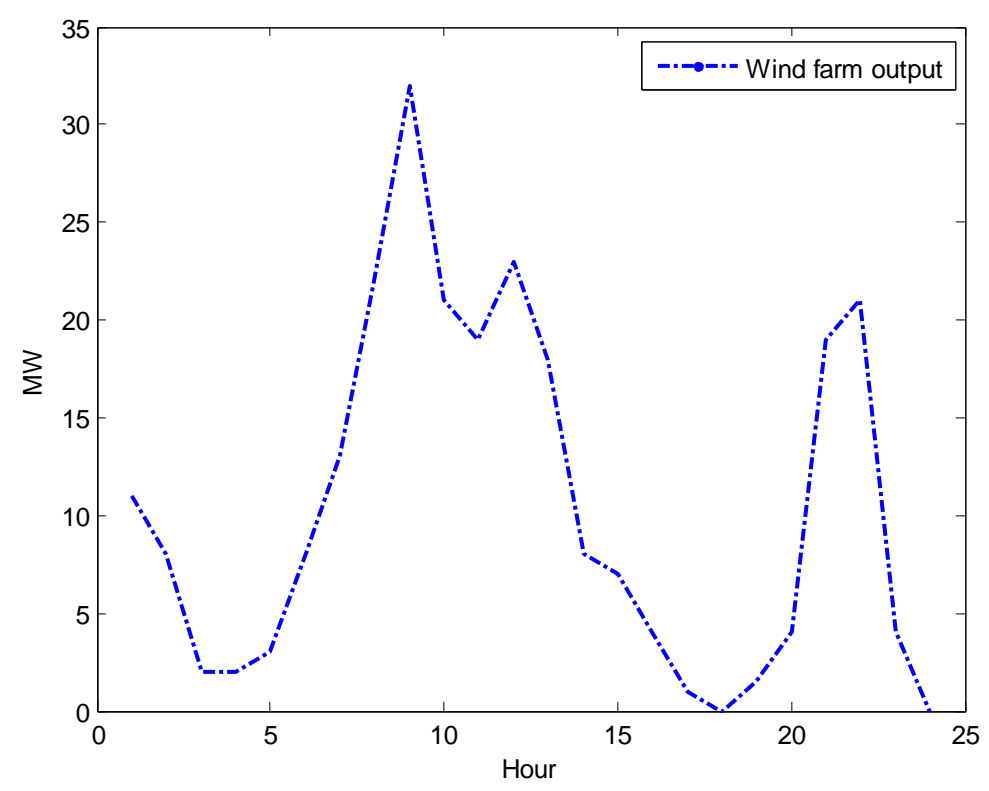

Fig.5-18 Forecasted Wind farm output

\subsubsection{Bidding Strategies with Wind Integration.}

A 24- hour optimal power flow solution is obtained in MATPOWER for the system with the forecasted wind farm output. In the event of wind power availability, dispatching generators should reduce their outputs to accommodate the wind power in the energy market. The MCP with and without the windfarm is different and can be considered in two different ways in the competitive electricity market. One option is that the wind farms will not be charged the output variability penalty and will be allowed to bid into the market as other dispatchable generators. Another option, which is used in this research work, is to use the wind farm output as and when it is available. In this case the new MCP with windfarm is to be determined to take care of wind generation and variability of wind power. With proper pricing mechanism and the MCP determination, the efficiency of the market can be improved. Figure 519 shows that the presence of wind generation decreases the incremental cost of the online generators and thereby decreases the MCP. Wind energy, thus has a positive impact on customer benefit. 


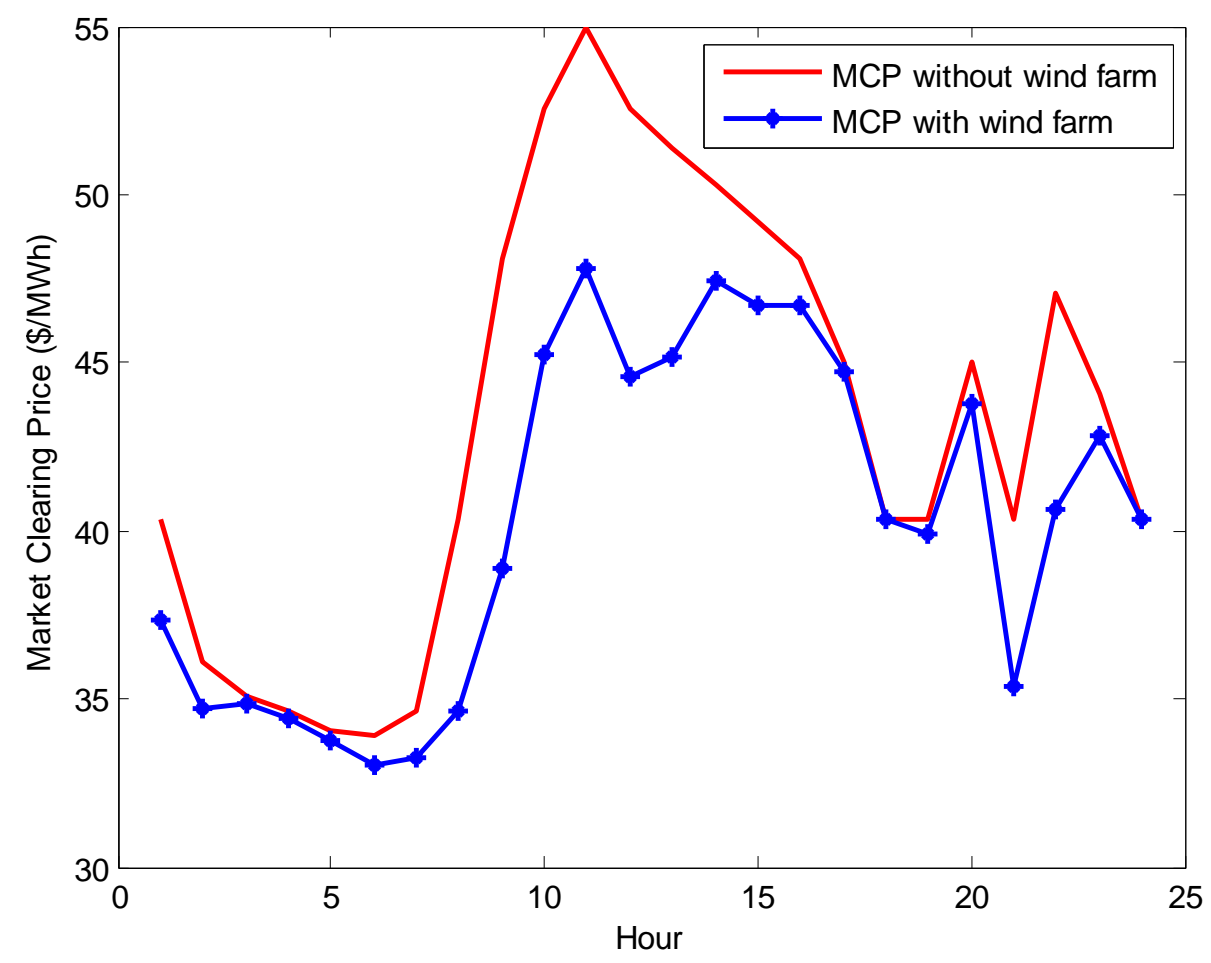

Fig.5-19 Variation of Market Clearing Price with Wind Integration.

With the determination of the new MCP, the PBUC optimization program is run to determine the optimal commitment schedule of the generators. To integrate the effects of uncertainty in wind availability, three simulated wind scenarios are created. Scenario I assumes low volatility in wind power for the forecasted wind output shown in Figure 5-19. Scenario II represents high volatility which follows a normal distribution with a standard deviation of $10 \%$. In Scenario III, the intermittency of wind power is considered during hours 17-20, when the wind power drops to zero. The simulated scenarios are in Table $5-8$.

Table 5-8 Wind Farm Data

\begin{tabular}{|c|c|c|c|c|}
\hline Hour & $\begin{array}{c}\text { Forecasted Wind } \\
\text { Power (MW) }\end{array}$ & $\begin{array}{c}\text { Wind Power } \\
\text { Scenario 1 } \\
\text { (MW) }\end{array}$ & $\begin{array}{c}\text { Wind Power } \\
\text { Scenario 2 } \\
\text { (MW) }\end{array}$ & $\begin{array}{c}\text { Wind Power } \\
\text { Scenario 3 } \\
\text { (MW) }\end{array}$ \\
\hline 1 & 11 & 9.75 & 9.35 & 11 \\
\hline 2 & 8 & 7.28 & 6.8 & 8 \\
\hline 3 & 2 & 1.78 & 1.7 & 2 \\
\hline 4 & 2 & 2 & 1.7 & 2 \\
\hline
\end{tabular}




\begin{tabular}{|c|c|c|c|c|}
\hline 5 & 3 & 3.07 & 2.55 & 3 \\
\hline 6 & 8 & 8.11 & 6.8 & 8 \\
\hline 7 & 13 & 11.97 & 11.05 & 13 \\
\hline 8 & 22 & 21.97 & 18.7 & 22 \\
\hline 9 & 32 & 30.35 & 27.2 & 32 \\
\hline 10 & 21 & 21.75 & 17.85 & 21 \\
\hline 11 & 19 & 18.14 & 16.15 & 19 \\
\hline 12 & 23 & 22.27 & 19.55 & 23 \\
\hline 13 & 18 & 18.23 & 15.3 & 18 \\
\hline 14 & 8 & 7.42 & 6.8 & 8 \\
\hline 15 & 7 & 7.54 & 5.95 & 7 \\
\hline 16 & 4 & 3.98 & 3.4 & 4 \\
\hline 17 & 1 & 1.02 & 0.85 & 0 \\
\hline 18 & 0 & 0 & 0 & 0 \\
\hline 19 & 1.5 & 1.4 & 1.275 & 0 \\
\hline 20 & 4 & 4.48 & 3.4 & 0 \\
\hline 21 & 19 & 22.16 & 16.15 & 19 \\
\hline 22 & 21 & 21.97 & 17.85 & 21 \\
\hline 23 & 4 & 3.91 & 3.4 & 4 \\
\hline 24 & 0 & 0 & 0 & 0 \\
\hline
\end{tabular}

\subsubsection{Dispatch with forecasted wind power}

With forecasted wind power given in Table III, PBUC determines the dispatch of the non-wind units as given in Table 5-9. The cheapest unit G2 is always - ON and the unit G1 is committed for Hours 11-23. With the availability of wind power, GENCO bids more energy and ancillary services in the market as shown in Figure 5-20. The commitment status of the two non wind units, G1 and G2 remain the same, however, they reduce their generations to accommodate wind power. It is noticed that from hours 1- 11, the GENCO bids more and from hours 12- 24 it maintains the bid at the previous value. At hour 12, since G1 turns -OF”, the energy bid with wind generation is less than the original bid. Figure 5-20 also shows the bidding changes for the ancillary services. The energy bids with wind are assumed to be contracted by the GENCO to the power pool in the day ahead market. 
Table 5-9 PBUC Plans for the Generators

\begin{tabular}{|c|c|c|c|}
\hline \multicolumn{2}{|c|}{ Scenario } & \multicolumn{4}{|c|}{ Hours (0-24) } \\
\hline $\begin{array}{c}\text { Forecasted Schedule } \\
\text { without wind }\end{array}$ & Unit G1 & 1000000000001111111111110 \\
\cline { 2 - 4 } & Unit G2 & 0111111111111111111111111 \\
\hline $\begin{array}{c}\text { Forecasted Schedule } \\
\text { with wind }\end{array}$ & Unit G1 & 1000000000001111111111110 \\
\cline { 2 - 4 } & Unit G2 & 0111111111111111111111111 \\
\hline Scenario 1 & Unit G1 & 1000000000001111111111110 \\
\cline { 2 - 4 } & Unit G2 & 0111111111111111111111111 \\
\hline Scenario 2 & Unit G1 & 1000000000000000000000000 \\
\cline { 2 - 4 } & Unit G2 & 0111111111111111111111111 \\
\hline Scenario 3 & Unit G1 & 1000000000001111111111110 \\
\cline { 2 - 4 } & Unit G2 & 0111111111111111111111111 \\
\hline
\end{tabular}

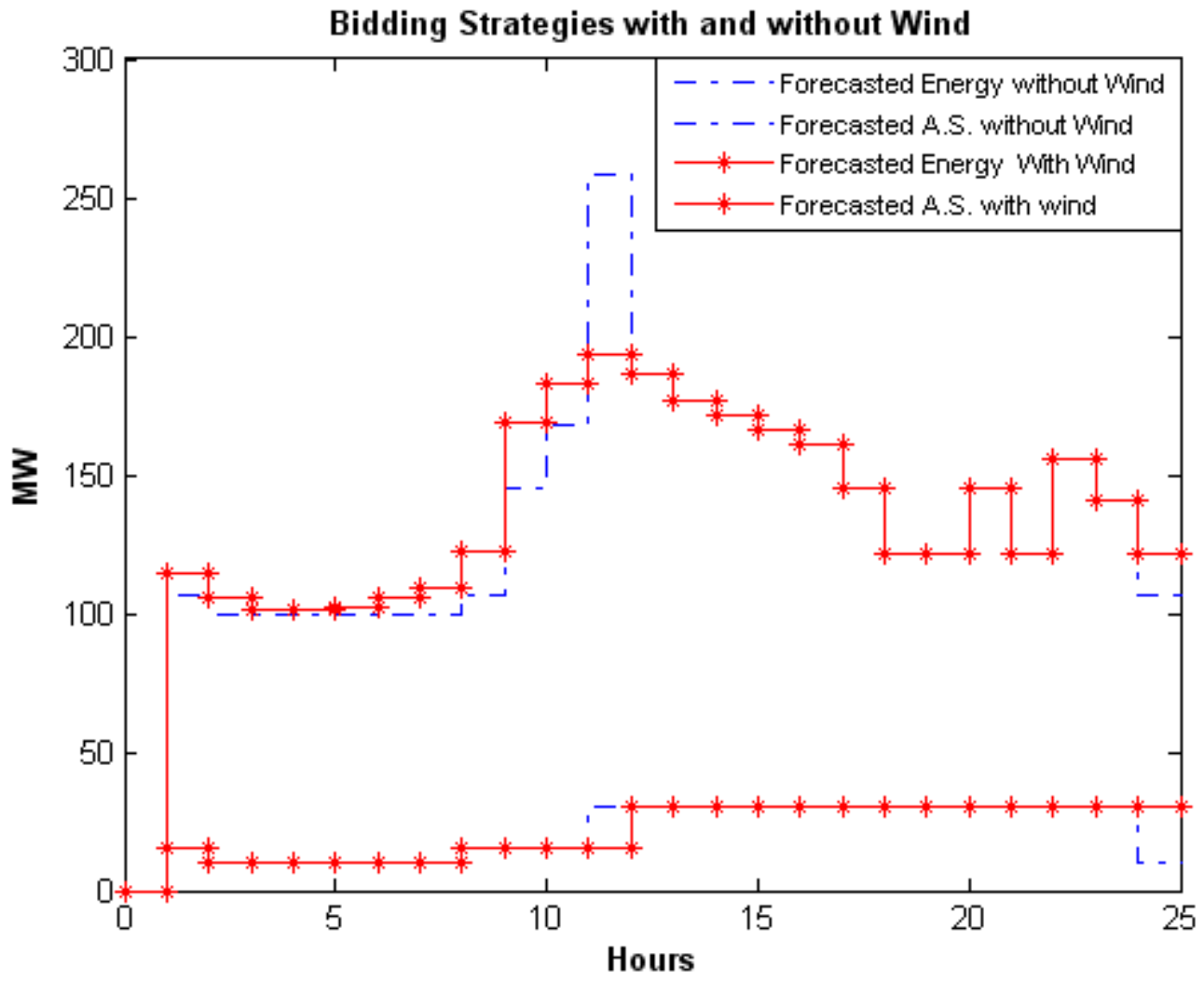

Fig.5-20 Bidding Strategy of the GENCO with and without wind. 


\subsubsection{Scenario I: Dispatch with low wind volatility.}

Scenario I, considers low volatility in forecasted wind power. This poses a challenge for the GENCO as changes in the expected wind power may require a re-dispatch from the non wind generators. The PBUC solution for this scenario in Table 5-9 shows that G2 is committed for 24 hours and the commitment of G1 is same as the forecast.

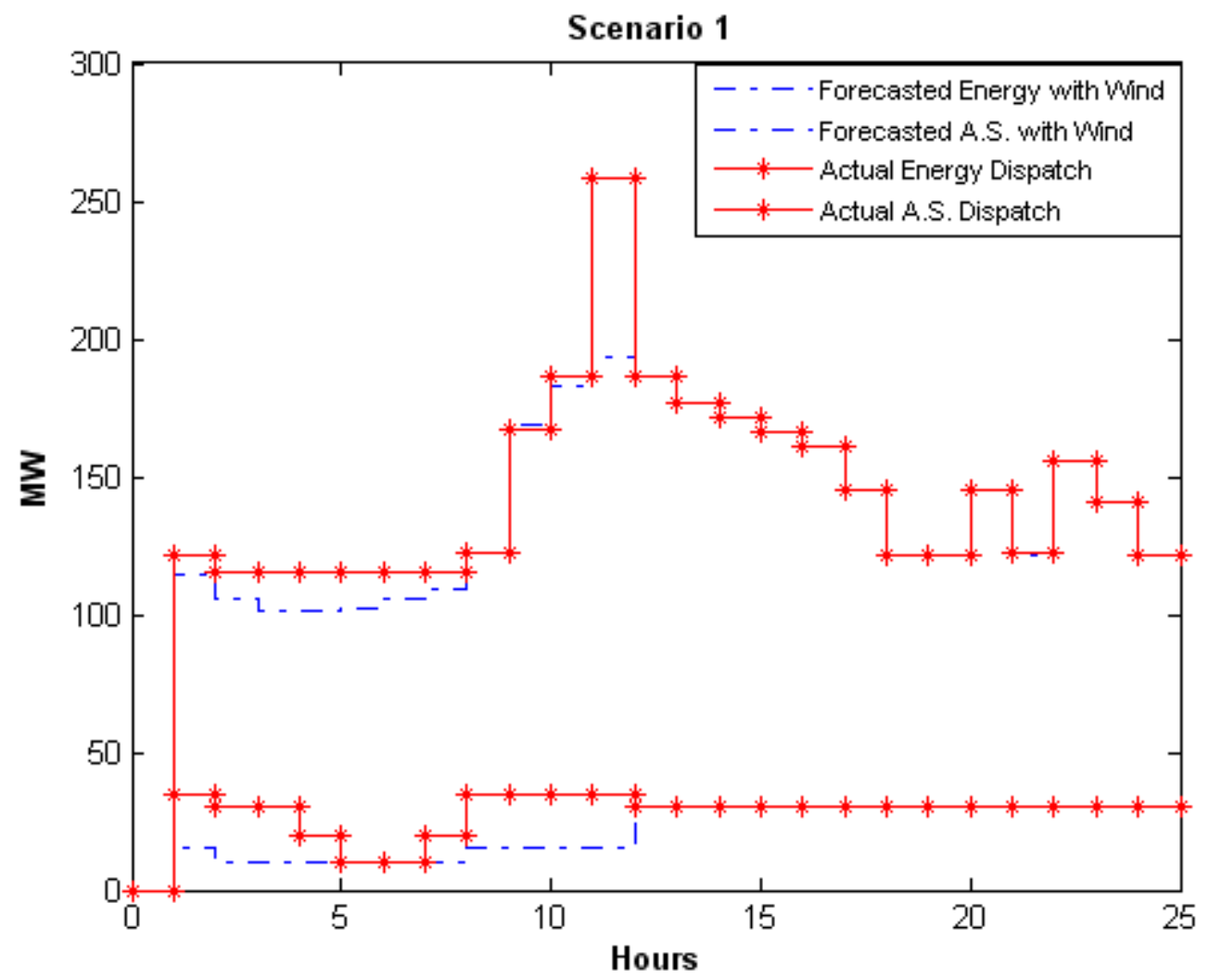

Fig.5-21 Bidding Strategy for Scenario I

Figure 5-21 shows the committed dispatch and the actual dispatch for Scenario I. It is noticed that, due to low volatility of the wind, the GENCO is able to satisfy its contract for hours 1-9 and hours 10-24. In hour 9, there is a marginal decrease in the dispatch from the committed value due to the ramp up and quick start constraints on the non-wind generators. The unit with a faster ramp rate, G1 has a quickstart of $5 \mathrm{MW}$. For hours 1-24, the ancillary services contract is satisfied by the GENCO.

\subsubsection{Scenario II: Dispatch with high wind volatility}

Scenario II considers high volatility in forecasted wind power due to incorrect forecast. The PBUC schedule in Table 5-9 indicates that unit G2 is - ON for 24 hours and the unit G1 is -OF" for 
hours 1-24 to maximize profit. Highly volatile wind generation results in the GENCO violating its contract to the power pool for hours 1-3 and hours 6-24 as shown in Figure 5-22 due to ramp up and quickstart constraints of the non-wind generators. In hours 3-6 the higher capacity unit G2 ramps up to meet the committed value. The violation of contract may lead to penalties imposed on the GENCO, leading to a sizeable reduction in their profits. The ancillary services contract is also violated for hours $12-24$.

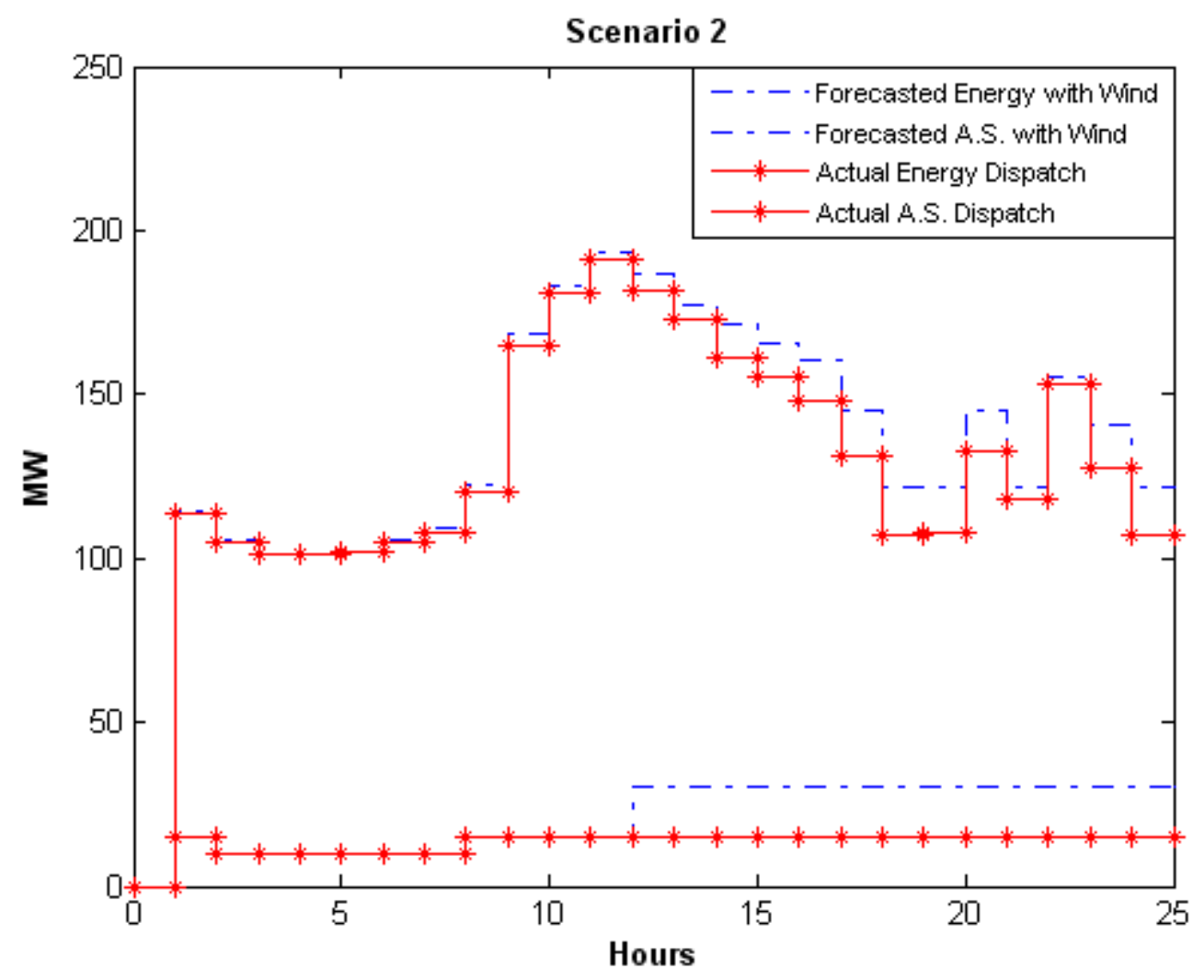

Fig.5-22 Bidding Strategy for Scenario II 


\subsubsection{Scenario III: Dispatch with wind intermittency.}

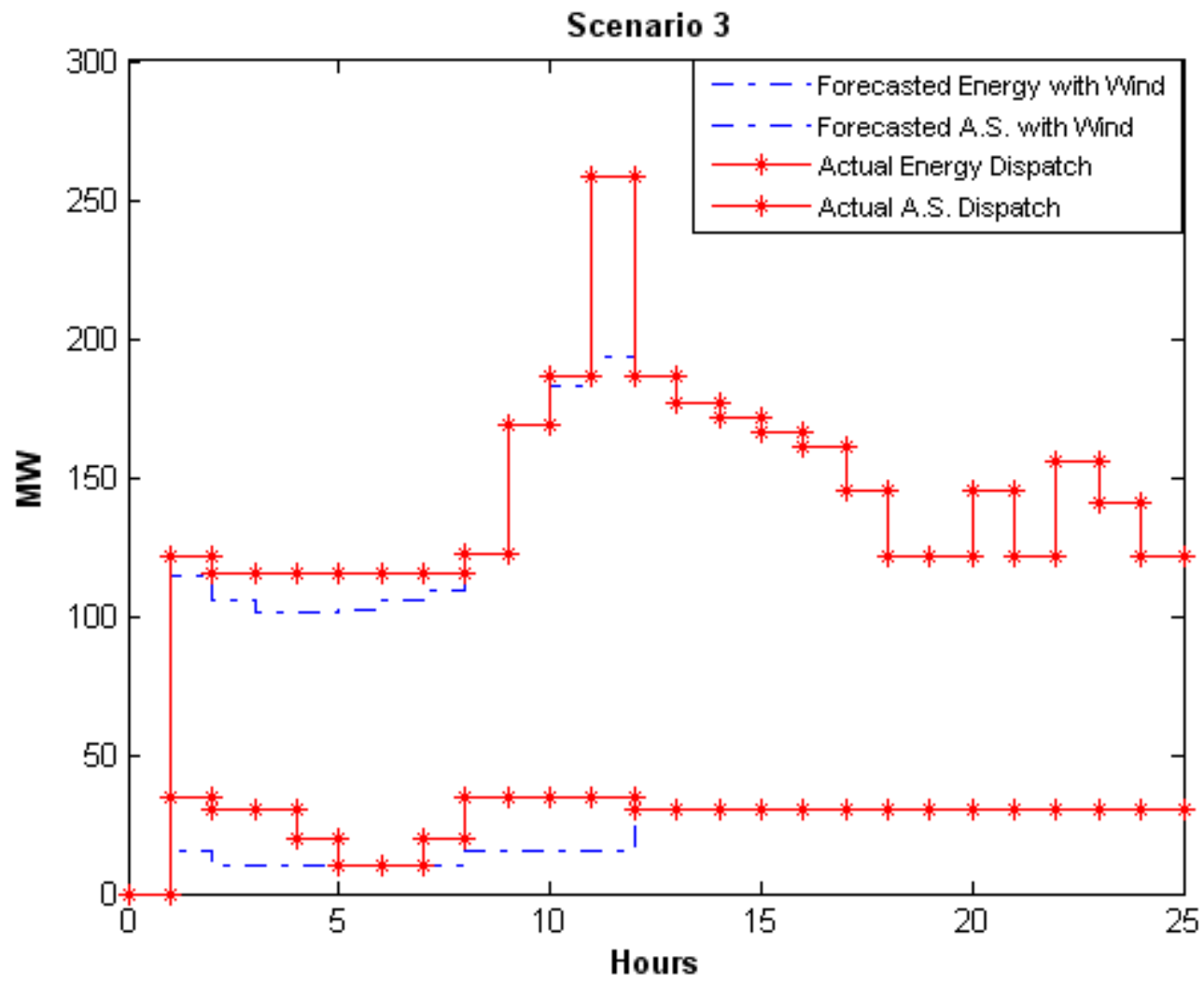

Fig.5-23 Bidding Strategy for Scenario III

Scenario III considers the effect of intermittency in forecasted wind power. The PBUC schedule in Table 5-9 shows that unlike Scenario II, the unit G1 is now turned - ON for hours 12- 23, while the unit G2 is -ON for hours 1-24. Figure 5-23 shows that the GENCO meets the contract during wind intermittency in hours 17-20 by ramping up G1 and G2.It is evident that in this scenario, the ramp and quickstart constraints of G1 and G2 are such that brief wind intermittency can be met by the GENCO and satisfy the contracted value. Ancillary services like spinning and non spinning reserve can be met by the GENCO, without violation of its contract due to brief periods of wind intermittency. From the three scenarios, it is evident that, the GENCO is able to dispatch its committed value under low volatility and brief intermittency and also maximizes its profit, however pays a penalty under highly volatile wind conditions. 


\section{Chapter 6}

\section{Conclusions and Future Work}

\subsection{Conclusions}

In this thesis a utility distribution model is developed in OpenDSS from an existing model in CYMDIST. The impacts of DG on distribution system voltage profiles and losses and power market operation have been analyzed. The conclusions drawn from both these works and scope for future work have been presented in this section.

\subsubsection{Distribution Feeder modeling}

In this thesis a utility distribution feeder model is developed in OpenDSS from the existing model in a commercial analysis tool. A detailed one-on-one parameter mapping approach is used to model every component in the system. The component models have been integrated to the feeder to run the power flow analysis. The results of the power flow solution obtained in OpenDSS for the two medium voltage feeders compare well to the results provided by the utility analysis tool. Three different models were used for the distributed load and a newer release of OpenDSS was used for accurate impedance models of the overhead line. The important conclusions of this work can be summarized as follows.

- OpenDSS is a highly scripted language and the syntax and formatting of information is very important for accurate analysis. At the earlier stages of this work, a lot of errors in models were due to the errors in formatting. A rigorous analysis of the script led to the correction of these errors.

- The modeling of distributed load has a great impact on the power flow results. The distributed load can be considered in three different ways: Spot load lumped at one third of the section; Spot load lumped at the midpoint of the section, two-thirds of the load at one fourth distance of the section and the remaining one third at the end of the feeder. Of these, lumping the load at the midpoint of the section achieved the most accurate power flow results.

- The modeling of overhead and underground lines was a challenging experience. The overhead lines were defined by the Line Geometry and the underground cables are defined by Line codes in symmetrical components. A detailed comparison of the impedances and susceptances of the 
overhead lines calculated by these software was done. It was found that the incorrect susceptances of the overhead line could lead to considerable KVAR losses. The issue of error in susceptance calculation was resolved in a newer release of OpenDSS.

- The regulator modeling was found to be different in both the software. The regulator was modeled as a line drop compensator in the utility analysis tool and it was modeled as a transformer in OpenDSS. To obtain accurate voltage results, a slight change was noticed in the power flow in the two systems.

Utilities are employing a number of engineering, enterprise and computational analysis tools for their distribution systems. The different features provided by various tools and vendors require utilities to conduct interoperable studies for their feeders. An efficient information exchange and sharing infrastructure is the key to its success. By developing the utility feeder model in OpenDSS from the existing model in a commercial analysis tool, this thesis explores the modeling complexities for distribution systems. The comparison of the power flow solutions in both these tools lead to a gap analysis and scope for improvement.

\subsubsection{Integration of PV system to the Distribution grid.}

In this thesis, a PV model was used to study the steady state integration issues of PV to the distribution grid. A PV model was developed in MATLAB/Simulink for interfacing with the IEEE 13 node feeder and the voltage profiles were studied for different levels of PV penetration to analyze the impacts. The results showed that at high PV penetration, there was a voltage unbalance and increase in the losses. Subsequently, an analysis was done on the utility feeder model developed. For this system a utility scale PV was required for simulation and penetration levels were varied from $10 \%$ to $30 \%$. Three different PV locations were considered namely: at the head of the feeder, at the tail of the feeder and utility specific locations. The utility scale PV model was defined as a generation load shape in OpenDSS with an interconnection transformer for various PV penetrations. The 24 hour time varying loads and PV generation was considered for this study. The results indicated a voltage unbalance at peak penetration at higher PV penetration levels for different location scenarios. A loss analysis of the feeder indicated higher feeder losses at hours with peak PV generation. The location of PV near to the load centers improved the power quality by reducing the losses in the line. Significant measures are to be taken by the utility to mitigate these impacts. The measures include changing the regulator and LTC tap settings, reconductoring distribution lines and changing the placement of capacitor banks. Utilities could also employ storage model to efficiently manage the dispatch of PV systems. 


\subsubsection{Impacts of DG on Market Operation}

The impacts of DG on power market operations were considered as a novel approach to the PBUC schedules of the Genco by integrating the effects of wind intermittency and market price variations. The results indicate that the profit of the Genco is largely dependent on the wind intermittency and volatility. From the three scenarios of wind intermittency, it is evident that, the Genco is able to dispatch its committed value under low volatility and brief intermittency and also maximizes its profit, however pays a penalty under highly volatile wind conditions. The results for the test system show that the physical limitations of the units such as ramping and quickstart are crucial for accommodating the volatility of the wind power. In a wind based power system a tradeoff between security and economy must be achieved such that the security of the system is maintained while the operational cost is minimized. Another option for accommodating wind power volatility is to allocate additional hourly reserves or utilize battery storage. The problem with this option is that the security of the power system may not be guaranteed since the system may not have enough ramping capabilities in real time and the battery may be bound by physical constraints.

\subsection{Future Work}

The distribution system, its modeling and analysis is gaining a vital importance with the advent of deregulation. A number of utilities are expressing interest in adopting Distributed generation into their infrastructure and solar photovoltaics have been found to be a viable option. The accurate modeling and analysis of the impacts of DG requires specialized tools and algorithms. There is an urgent need for systemic interconnection studies due to the proliferation of the utility scale DG and high PV penetration scenarios. This leads to important future research directions in this area to be explored by all stakeholders. Some of the important ones are summarized below.

- The studies need to cover the various steady state and dynamic impacts of distribution feeder operation and under the new generation and load regime. A major obstacle for this study is the lack of high resolution DG and load data specific to the study area for an extended period of time. This need has been realized by many utilities and currently work is under way to install and enhance monitoring and data collection equipment. 
- The modeling and design of cost effective storage could be the game changer for PV integration. An effective storage technology could dispatch the PV in an optimized way. The optimization of PV and storage for effective energy management is an area of interest.

- The design of smart inverters with communication and Volt/VAR control capabilities is sought after by the industry. The interaction of the inverters with utility voltage control equipments such as regulators and LTC's can prevent islanding scenarios and reduce the impact of PV on voltage stability.

- A lot of interest is being generated over coordinating Plug in Hybrid electric vehicles with PV generation. With proper coordination, the PHEV's could be used as a storage source for PV generation, during the day time, and can be used to supply the energy back under non -PV producing hours. This requires smart optimization algorithms and design of communication capabilities for the PHEV. 


\section{REFERENCES}

[1] Fitle XIII - Smart Grid, Sec. 1301, Statement of Policy on Modernization of Electricity Grid", Energy Independence and Security Act of 2007 (EISA), USA.

[2] Smart Grid: An Introduction”, Program Concept paper, U.S. Department of Energy, 2001.

[3] Rabinowitz, M., - Powr Systems of the Future”, IEEE Power Engineering Review, 2000.

[4]Kersting, W., H., Đistribution System Modeling and Analysis”, Boca Raton, FL: CRC, 2002.

[5] Chen, C., Zhu, Y., Xu, Y., —Dissibuted generation and Demand Side Management”, Proceedings of the 2010 China International Conference on Electricity Distribution (CICED), 13-16 Sept. 2010, pp: 1-5.

[6] Golkar, M., A., -Disibuted Generation and competition in electric distribution market", Proceedings of the 2009 IEEE EUROCON, 18-23 May 2009, pp: 558-563.

[7] Guan, F., H., Zhao, D., M., Zhang, X., Shan, B., T., Liu, Z., -Resarch on distributed generation technologies and its impacts on power system", Proceedings of the International Conference on Sustainable Power Generation and Supply, 6-7 April 2009, pp: 1-6.

[8] Driesen, J., Belmans, R., —Disibuted generation: challenges and possible solutions", Proceedings of the 2006. IEEE Power Engineering Society General Meeting, 2006, pp: 1-8.

[9] Modern Grid Benefits", Report by National Energy Technology Laboratory (NETL) and U.S. Department of Energy, August 2007.

[10] Nowak, S., — Tends in Photovoltaic Applications: Survey report of selected IEA countries between 1992 and 2008", Proceedings of the International Energy Agency Photovoltaic Power Systems Program (IEA-PVPS), St. Ursen, 2009.

[11] Markvart, T., Castaner, L., Practical Handbook of Photovoltaics, Fundamentals and Applications", Elsevier, 2003.

[12] Basso, T.S., DeBlasio, R., -HEE 1547 series of standards: interconnection issues," IEEE Transactions on Power Electronics, Vol.19, No. 5, Sept. 2004, pp: 1159- 1162. 
[13] Khushalani, S., Schulz, N., N., —Unbalnced distribution power flow with distributed generation," Proceedings of the IEEE Transmission and Distribution Conference, Dallas, TX, May 2006.

[14] Shahidehpour, M., Yamin, H.; Li, Z., Market Operations in Electric Power Systems: Forecasting, Scheduling, and Risk Management”, John Wiley \& Sons, Inc., CRC 2002.

[15] Kangpeng, P., The research for network model of distribution management system", Proceedings of the 2010 China International Conference on Electricity Distribution (CICED), 13-16 Sept. 2010, pp: 15.

[16] Nazarko, J., - Mddeling of electrical power distribution systems by application of experimental design", Proceedings of the IEEE Power Engineering Society Winter Meeting, 2000, pp: 1145-1149.

[17] Rudnick, H., Munoz, M., Influence of modeling in load flow analysis of three phase distribution systems", Proceedings of the IEEE Colloquium in South America, 1990, pp: 173-176.

[18] Miu, K., Kleinberg, M., Impact studies of unbalanced multi-phase distribution system component models", Proceedings of the IEEE Power and Energy Society General Meeting, 25-29 July 2010, pp: 1-4.

[19] Schneider, K.P., Fuller, J.C., Đetailed end use load modeling for distribution system analysis", Proceedings of the IEEE Power and Energy Society General Meeting, 25-29 July 2010, pp: 1-7.

[20] Lind, R., Karlsson, D., Đistribution system modeling for voltage stability studies", IEEE Transactions on Power Systems, Vol.11, No.4, Nov. 1996, pp: 1677-1682.

[21] Program on Technology Innovation: Distribution Common Information Model (CIM) Modeling of Two North American Feeders". EPRI, Palo Alto, CA: 2009. 1018281.

[22] Wang, X., Schulz, N.N., Neumann, S., - CIM extensions to electrical distribution and CIM XML for the IEEE radial test feeders", IEEE Transactions on Power Systems, Vol.18, No.3, Aug. 2003, pp: 10211028.

[23] Lambert, E., -CDPSM: Common distribution power system model: When, why, what, how, who?", Proceedings of the IEEE/PES Power Systems Conference and Exposition (PSCE), 20-23 March 2011. pp: $1-10$. 
[24] Kojovic, L., Modeling of DG to interface with distribution system”, Proceedings of the IEEE Power Engineering Society Summer Meeting, Vol.1, 25-28 July 2002, pp.179-180.

[25] Khushalani, S., Solanki, J.M., Schulz, N.N., Đevelopment of Three-Phase Unbalanced Power Flow Using PV and PQ Models for Distributed Generation and Study of the Impact of DG Models", IEEE Transactions on Power Systems, Vol.22, No.3, Aug 2007, pp:1019-1025.

[26] Hussein, D.N., El-Syed, M., Attia, H.A., Modeling and simulation of distributed generation (DG) for distribution systems load flow analysis", Proceedings of the Eleventh International Middle East Power Systems Conference, Vol.1, 19-21 Dec. 2006, pp: 285-291.

[27] Xiao-bo, T., Guo-qing, T., Power Flow for Distribution Network with Distributed Generation", Proceedings of the 2010 Asia-Pacific Power and Energy Engineering Conference (APPEEC), 28-31 March 2010, pp: 1-4.

[28] Wenyu, Y., Xuying, Y., Jiandong, D., Xiaozhong, W., Yue, F., Power flow calculation in distribution networks containing distributed generation", Proceedings of the 2008 China International Conference on Electricity Distribution (CICED), 10-13 Dec. 2008, pp: 1-5.

[29] Chen, H., Chen, J., Shi, D., Duan, X., Power flow study and voltage stability analysis for distribution systems with distributed generation", Proceedings of the2006 IEEE Power Engineering Society General Meeting, pp: 1-8.

[30] Ding, M., Guo, X., Zhang, Z., Three Phase Power Flow for Weakly Meshed Distribution Network with Distributed Generation", Proceedings of the 2009 Asia-Pacific Power and Energy Engineering Conference, 27-31 March 2009, pp.1-7.

[31] Xue, Y., Deng, Y., Ma, S., Power flow control of a distributed generation unit in micro-grid", Proceedings of the IEEE 6th International Power Electronics and Motion Control Conference, 17-20 May 2009, pp: 2122-2125.

[32] Okada, N., Kobayashi, H., Takigawa, K., Ichikawa, M., Kurokawa, K., Loop power flow control and voltage characteristics of distribution system for distributed generation including PV system", Proceedings of 3rd World Conference on Photovoltaic Energy Conversion, Vol.3, 18 May 2003, pp: 2284-2287. 
[33] Dai, M., Marwali, M.N., Jung, J.W., Keyhani, A., Power Flow Control of a Single Distributed Generation Unit”, IEEE Transactions on Power Electronics, Vol.23, No.1, Jan. 2008, pp: 343-352.

[34] Shugar, D.S., - Phtovoltaics in the utility distribution system: The evaluation of system and distributed benefits", Proceedings of the Twenty First IEEE Photovoltaic Specialists Conference, Vol.2, 21-25 May 1990, pp: 836-843.

[35] Lambeth, R., Lepley, T., - Bstributed photovoltaic system evaluation by Arizona Public Service Company," Proceedings of the Twenty Third IEEE Photovoltaic Specialists Conference, 10-14 May 1993, pp: $14-20$.

[36] Kai, Z., Agalgaonkar, A.P., Muttaqi, K.M., Perera, S., Browne, N., -Supprt of distribution system using distributed wind and PV systems," Proceedings of the Australasian Universities Power Engineering Conference, 27-30 Sept. 2009, pp: 1-6.

[37] Mustachhi, C., Cena, V., Rochhi M., Stochastic Simulation of Hourly Global Radiation Sequences", Proceedings of the Australasian Universities Power Engineering Conference, 27-30 Sept. 2009, pp: 1-6.

[38] Wu, J., Chan, C., Loh, J., Choo, F., Chen, L.H., -Sølr radiation prediction using statistical approaches", Proceedings of the 7th International Conference on Information, Communications and Signal Processing, 8-10 Dec. 2009,pp.1-5.

[39] Iqdour, R., Zeroual, A., - Arule based fuzzy model for the prediction of daily solar radiation", Proceedings of the 2004 IEEE International Conference on Industrial Technology, Vol.3, 8-10 Dec. 2004, pp. 1482- 1487.

[40] Momoh, J.A., Wang, Y., Eddy-Posey, F., -Optnal power dispatch of photovoltaic system with random load", Proceedings of the 2004 IEEE Power Engineering Society General Meeting, Vol.2, 10-10 June 2004, pp: 1939-1945.

[41] Giraud, F., Salameh, Z.M., -Aalysis of the effects of a passing cloud on a grid-interactive photovoltaic system with battery storage using neural networks", IEEE Transactions on Energy Conversion, Vol.14, No.4, Dec 1999, pp: 1572-1577. 
[42] Zahedi, A., - Anew approach in size optimization and performance prediction of photovoltaic-hybrid power systems", Proceedings of the Twenty-Eighth 2000 IEEE Photovoltaic Specialists Conference, pp: 1548-1551.

[43] Daming, X., Longyun, K., Liuchen, C., Binggang, C., —Optnal sizing of standalone hybrid wind/PV power systems using genetic algorithms", Proceedings of the 2005 Conference on Electrical and Computer Engineering, 1-4 May 2005, pp: 1722-1725.

[44] Hancock, M., Outhred, H.R., Kaye, R.J., - Anew method for optimizing the operation of stand-alone PV hybrid power systems", Proceedings of the IEEE Photovoltaic Specialists Conference, Vol.1, 5-9 Dec 1994, pp: 1188-1191.

[45] Djarallah, M., Azoui, B., - Gr Connected Interactive Photovoltaic Power Flow Analysis: A Technique for System Operation Comprehension and Sizing”, Proceedings of the 41st International Universities Power Engineering Conference, Vol.1, 6-8 Sept. 2006, pp: 69-73.

[46] Molina, M.G., Mercado, P.E., - Mdeling and control of grid-connected photovoltaic energy conversion system used as a dispersed generator", Proceedings of the IEEE Transmission and Distribution Conference and Exposition: Latin America, 13-15 Aug. 2008, pp: 1-8.

[47] Bzura, J.J., The New England electric photovoltaic systems research and demonstration project," IEEE Transactions on Energy Conversion, Vol. 5, No.2, Jun. 1990, pp. 284-289.

[48] Abouzahr, I., Ramakumar, R., -Anapproach to assess the performance of utility-interactive photovoltaic systems", IEEE Transactions on Energy Conversion, Vol. 8, No. 2, Jun. 1993, pp: 145-153.

[49] Haeberlin, H., -Eolution of inverters for grid connected PV systems from 1989 to 2000", Proceedings of the IEEE Photovoltaic Specialists Conference, 2001, pp: 426-430.

[50] Carrasco, J.M., Franquelo, L. G., Bialasiewicz, J.T., Galvan, E., Guisado, R.C.P., Prats, M.A.M., Leon, J.I., Moreno-Alfonso, N., -Bwer electronic systems for the grid integration of renewable energy sources: A survey," IEEE Transactions on Industrial Electronics, Vol. 53, No. 4, Aug. 2006, pp: 10021016. 
[51] Salas, V., National Survey Report of PV Power Applications in Spain, 2008", Report by International Energy Agency, Photovoltaic Power Systems Program and Universidad Carlos III de Madrid. Available: http://www.iea-pvps.org.

[52] Keesee, M., Newmiller, J., Whitaker, C., Impact of distributed solar on SMUD'S peak load and local distribution system", Proceedings of the IEEE Photovoltaic Specialists Conference, 11-16 May 2008, pp: 1-6.

[53] Liu, Y., Bebic, J., Kroposki, B., de Bedout, J., Ren, W., Đistribution System Voltage Performance Analysis for High-Penetration PV", Proceedings of the IEEE Energy 2030 Conference, 17-18 Nov. 2008, pp: $1-8$.

[54] Srisaen, N., Sangswang, A., Effects of PV Grid-Connected System Location on a Distribution System”, Proceedings of the IEEE Asia Pacific Conference on Circuits and Systems, 4-7 Dec. 2006, pp: $852-855$.

[55] Tonkoski, R., Lopes, L.A.C., $\forall$ oltage Regulation in Radial Distribution Feeders with High Penetration of Photovoltaics", Proceedings of the IEEE Energy 2030 Conference, 17-18 Nov. 2008, pp: $1-7$.

[56] Conti, S., Raiti, S., Tina, G., Vagliasindi, U., Study of the impact of PV generation on voltage profile in LV distribution networks", Proceedings of the 2001 IEEE Power Tech Conference, Vol.4, 2001, pp: 1-6.

[57] Hsieh, W.L., Lin, C.H., Chen, C.S., Hsu, C.T., Ku, T.T., Tsai, C.T., Ho, C.Y., Impact of PV generation to voltage variation and power losses of distribution systems", Proceedings of the 4th International Conference on Electric Utility Deregulation and Restructuring and Power Technologies, 69 July 2011, pp: 1474-1478.

[58] Katiraei, K.F., Agüero, J.R., Solar PV Integration Challenges”, IEEE Power and Energy Magazine, Vol.9, No.3, May-June 2011, pp: 62-71.

[59] Weidong, Y., Xia, Z., Feng, X., Impacts of Large Scale and High Voltage Level Photovoltaic Penetration on the Security and Stability of Power System", Proceedings of the 2010 Asia-Pacific Power and Energy Engineering Conference, 28-31 March 2010, pp: 1-5. 
[60] Structure of distribution grid and impacts of the solar electricity generation", Report by International Energy Agency, Photovoltaic Power Systems Program. Available: http://www.iea-pvps.org.

[61] Parameechok, R., Premrudeepreechacharn, S., Kasirawat, T., Impacts of photovoltaic power generation on very long distribution line", Proceedings of the North American Power Symposium (NAPS), 4-6 Oct. 2009, pp: 1-5.

[62] Enslin, J.H.R., Network impacts of high penetration of photovoltaic solar power systems", Proceedings of the IEEE Power and Energy Society General Meeting, 25-29 July 2010, pp: 1-5.

[63] Senjyu, T., Yamashiro, H., Shimabukuro, K., Uezato, K., Funabashi, T., -Fatssolution technique for large-scale unit commitment problem using genetic algorithm", Proceedings of IET Generation, Transmission and Distribution, Vol. 150, Issue 6,12 Nov 2003, pp: 753-760.

[64] Mantawy, A.H., Abdel-Magid, Y.L. and Selim, S.Z., - Anew genetic algorithm approach for unit commitment", Proceedings of the International Conference on Genetic Algorithms in Engineering Systems: Innovations and Applications, 2-4 Sept 1997, pp: 215-220.

[65] Pokharel, B.K., Shrestha, G.B., Lie, T.T., and Fleten, S. E., -Fice based unit commitment for Gencos in deregulated markets", Proceedings of the IEEE Power Engineering Society General Meeting, 12-16 June, 2005, pp: 2159-2164.

[66] Li, T., and Shahidehpour, M., - Flce based unit commitment: A case of Lagrangian relaxation versus mixed integer programming", IEEE Transactions on Power Systems, Vol. 20, No. 4, November 2005, pp: 2015-2025.

[67] Attaviriyanupap, P., Kita, H., Tanaka, E., and Hasegawa, J., - Ahybrid LR-EP for solving new profit based UC problem under competitive environment", IEEE Transactions on Power Systems, Vol. 18, No. 1, Feb. 2003, pp: 229-237.

[68] Xiaohui, Y., Yanbin, Y., Cheng, W. and Xiaopan, Z., - Arimproved PSO approach for profit based unit commitment in electricity market”, Proceedings of the IEEE/PES Asia and Pacific Transmission and Distribution conference \& Exhibition, Dalian, China, 2005. 
[69] Watanabe, I., Yamaguchi, N., Shiina, T. and Kurihara, I., -Agnt-based simulation model of electricity market with stochastic unit commitment", Proceedings of the International Conference on Probabilistic Methods Applied to Power Systems, 12-16 Sept 2004, pp: 403-408.

[70] Yu, J., Zhou, J., Wu, W. and Yang, J., —Soltion of the profit-based unit commitment problem by using the multi agent system", Proceedings of the $5^{\text {th }}$ World Congress on Intelligent Control and Automation, 15-19 June, 2004, Hangzhou, China.

[71] Fabbri, A., GomezSanRoman, T., RivierAbbad, J., MendezQuezada, V.H., Assessment of the Cost Associated With Wind Generation Prediction Errors in a Liberalized Electricity Market", IEEE Transactions on Power Systems, Vol. 20, No. 3, Aug. 2005, pp: 1440- 1446.

[72] Wind Energy Manual, 2002, Iowa Energy Center, USA.

Available: http://www.energy.iastate.edu/renewable/wind/wem/wem-02_toc.html.

[73] Spencer, R., - Utapped potential of wind power", IEEE Power Engineering Review, Vol. 22, No. 9, Sep. 2002, pp: 10-11.

[74] Milligan, M., Porter, K., -Detrmining the capacity value of wind: A survey of methods and implementation", Proceedings of Wind Power, Denver, 2005.

[75] Sideratos, G., Hatziargyriou, N.D., -An advanced statistical method for wind power forecasting", IEEE Transactions on Power Systems, Vol. 22, No. 1, Feb 2007.

[76] Kariniotakis, G., Waldl, I.H.P., Marti, I., Giebel, G., Nielsen, T.S., Tambke, J., Usaola, J., Dierich, F., Bocquet, A. and Virlot, S., -Nexgeneration forecasting tools for the optimal management of wind generation", Proceedings of the International Conference on Probabilistic Methods Applied to Power Systems, 11-15 June 2006, pp: 1-6.

[77] DeMeo, E., A., Grant, W., Milligan, M., R., Schuerger, M., J., - Whd plant integration: Cost, status \& issues," IEEE Power and Energy Magazine, Vol. 3, No. 6, Nov/Dec 2005, pp: 38-46.

[78] Zeineldin, H.H.; El-Fouly, T.H.M.; El-Saadany, E.F.; Salama, M.M.A., "Impact of wind farm integration on electricity market prices," Renewable Power Generation, IET, Vol.3, No.1, March 2009, pp.84-95. 
[79] Sinha, A., Basu, A.K., Lahiri, R.N., Chowdhury, S., Chowdhury, S.P., Crossley, P.A., -SÆing of market clearing price (MCP) in microgrid power scenario", Proceedings of the IEEE Power and Energy Society General Meeting - Conversion and Delivery of Electrical Energy in the 21st Century, 20-24 July 2008, pp:1-8.

[80] Singh, S.N., Erlich, I., - Stategies for Wind Power Trading in Competitive Electricity Markets", IEEE Transactions on Energy Conversion, Vol. 23, No. 1, March 2008.

[81] Streiffert, D., Philbrick, R. and Ott, A., - Amixed integer programming solution for market clearing and reliability analysis", Proceedings of the Power Engineering Society General Meeting, Vol. 3, June 2005.

[82] Yan, H., Luh, P.B., A fuzzy optimization method for integrated power system scheduling and inter utility power transaction with uncertainties", IEEE Transactions on Power Systems, Vol. 12, No. 2, May 1997.

[83] Venkatesh, B., Yu, P., Gooi, H.B., -Fuzzy MILP unit commitment incorporating wind generators”, IEEE Transactions on Power Systems, Vol. 23, No. 4, Nov 2008.

[84] Open DSS Manual, Electric Power Research Institute, July 2010.

[85] CYMDIST, Available: http://www.cyme.com/software/cymdist/

[86] The Java Tutorial, Available: http://download.oracle.com/javase/tutorial/

[87] JExcel API, Available: http://jexcelapi.sourceforge.net/

[88] The Eclipse Foundation Open Source Project, Available: http://www.eclipse.org/

[89] The Math Works ${ }^{\mathrm{TM}}$, Available: http://www.mathworks.com/products/matlab/description1.html

[90] "PV Module/ Simulink Models", ECEN 2060, University of Colorado at Boulder, Spring 2008.

[91] Simulink- Simulation based product design, Available: www.mathworks.com/products/simulink/

[92] "Grid Connected PV Systems", ECEN 2060, University of Colorado at Boulder, Spring 2008. 
[93] Shahidehpour, M., Yamin, H.; Li, Z., Market Operations in Electric Power Systems: Forecasting, Scheduling, and Risk Management”, John Wiley \& Sons, Inc., CRC 2002.

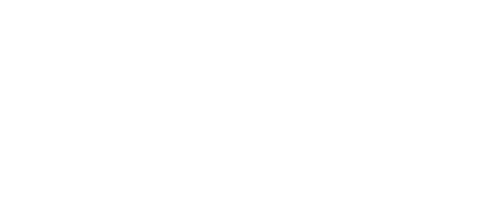

